
A Thesis
entitled

Design and Implementation of a Customized Encryption Algorithm for

Authentication and Secure Communication between Devices

by

\title{
Bhavana Daddala
}

Submitted to the Graduate Faculty as partial fulfillment of the requirements for the Master of Science Degree in Engineering

Dr. Hong Wang, Committee Chair

Dr. Ahmad Y. Javaid, Committee Co-Chair

Dr. Mansoor Alam, Committee Member

Dr. Amanda Bryant-Friedrich, Dean College of Graduate Studies

The University of Toledo

August 2017 
Copyright 2017, Bhavana Daddala

This document is copyrighted material. Under copyright law, no parts of this document may be reproduced without the expressed permission of the author. 
An Abstract of

Design and Implementation of a Customized Encryption Algorithm for Authentication and Secure Communication between Devices

by

\section{Bhavana Daddala}

Submitted to the Graduate Faculty as partial fulfillment of the requirements for the Master of Science Degree in Engineering

The University of Toledo

August 2017

Security is one of the biggest concerns in the developing world. It is important to ensure a safe transfer of information between communicating parties, protecting them from attacks. Many standards and developed encryption protocols are available as resources and are used based on the requirements. In this thesis, we propose a customized encryption algorithm and an authentication scheme to safely transfer information. The algorithm is a variation of Advanced Encryption Standard (AES) and is carried out between multiple devices. AES uses only one private key (symmetric key) to encrypt the data. The implementation works on a single standard irreducible polynomial of degree ' 8 ' which is further used to compute multiplicative inverse tables, S-boxes and inverse S-Boxes required for the working of every layer in the algorithm. As compared to AES, we use sixteen irreducible polynomials of degree ' 8 ' instead of one in our implementation. Key sizes for AES are usually 128, 192 and 256 bits in size; we use a 128-bit key. Unlike symmetric encryption, asymmetric encryption uses two keys, private and public keys. The public keys are shared among the communicating parties, while the private keys are kept secret. The keys are supposed to be large in size to maintain strength. Thus, they usually range from 512 bits to 2048 bits or more for asymmetric cryptography. The communicating parties can start with public-private keys and agree upon a common key (session key) which can 
be used as a key to AES. Diffie-Hellman key exchange protocol uses a combination of AES and RSA algorithms resulting in reliable cryptosystems. Our algorithm focuses on devising a new protocol for key establishment and agreement, using the combination framework. The implementation of the communication protocol between two devices, with a prospect of working with multiple devices using a centralized server, is presented. The outcome is to establish a different approach towards encryption and enhance security by providing protection against Man-in-the-Middle attacks. The customized algorithms are implemented using Python. 
To my family and friends for their love, endless support and encouragement. 


\section{Acknowledgments}

I wish to express my deepest gratitude to my advisor Dr. Hong Wang and coadvisor Dr. Ahmed Javaid for their continued support, guidance and encouragement, without which it would not have been possible to succeed in my research. I would like to thank graduate program director Dr. Mansoor Alam, for giving me an admission in Master's program at University of Toledo. The financial support from the EECS Department in the form of Tuition Scholarship and from the ET Department in the form of graduate/teaching assistantship is gratefully acknowledged. I would like to thank my lab mates Mohammad Rahat Helal, Niyaz Quamar for helping me in my research study.

Special thanks to my brother Raman Teja Venigalla for providing me the learning

resources to complete my thesis. Above all, I wish to express my deep appreciation to my family and friends who with their love and encouragement have made all this possible. 


\section{Contents}

Abstract

Acknowledgments $\quad$ vi

Contents vii

List of Tables $\quad \mathrm{x}$

List of Figures $\quad$ xii

List of Abbreviations $\quad$ xiv

1 Introduction 1

1.1 Problem Statement . . . . . . . . . . . . . . . . . . . . 1

1.2 Proposed Research Approach . . . . . . . . . . . . . . . . 2

1.3 Thesis Organization . . . . . . . . . . . . . . . . 2

2 Literature 4

2.1 Introduction . . . . . . . . . . . . . . . . 4

2.2 Background and Motivation ................ 4

2.3 Classifications . . . . . . . . . . . . . . . . 6

2.3.1 Symmetric Cryptography . . . . . . . . . . . . . . 7

2.3.2 Asymmetric Cryptography . . . . . . . . . . . . . . . . 10

2.4 Diffie Hellman Key Exchange (DHKE) . . . . . . . . . . . . . . . . . 14

2.5 Digital Signatures . . . . . . . . . . . . . . . . 15 
3 Standard Encryption Approach $\quad 18$

3.1 AES Encryption . . . . . . . . . . . . . . . . . 18

3.2 Galois Fields . . . . . . . . . . . . . . . . . . . . . . . 19

3.2.1 Multiplication in Galois Fields . . . . . . . . . . . . . . 20

3.2 .2 Inversion in Galois Fields . . . . . . . . . . . . . . . . . . . . . 21

3.3 Structure of AES . . . . . . . . . . . . . . . . . . . . . . . 22

3.3.1 Byte Substitution Layer . . . . . . . . . . . . . . . . . . . . 22

3.3.2 ShiftRows Layer . . . . . . . . . . . . . . . . . . . . . 25

3.3.3 MixColumn Layer . . . . . . . . . . . . . . . . . . . 25

3.3.4 Key Addition Layer . . . . . . . . . . . . . . . . 26

3.4 AES Decryption . . . . . . . . . . . . . . . . . 27

3.4.1 Inverse MixColumn Sublayer . . . . . . . . . . . . . . . . . . 28

3.4.2 Inverse ShiftRows Sublayer . . . . . . . . . . . . . . . . . . . . 29

3.4.3 Inverse Byte Substitution Layer . . . . . . . . . . . . . . . . . 29

4 Customized Encryption Approach 31

4.1 Introduction . . . . . . . . . . . . . . . . . 31

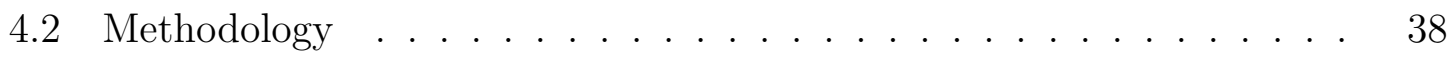

4.3 Communication Protocol . . . . . . . . . . . . . . . . . . . 40

4.4 Authentication Protocol . . . . . . . . . . . . . . . . . 42

5 Performance and Implementation $\quad 45$

5.1 Security Analysis . . . . . . . . . . . . . . . . 45

5.2 Algorithm Implementation . . . . . . . . . . . . . . . 47

5.2.1 Key Generation . . . . . . . . . . . . . . . . . 47

5.2.2 Hash Generation . . . . . . . . . . . . . . . . . . 47 
5.2.3 Encryption and Decryption _.............. . . 48

5.2.4 Gate Function . . . . . . . . . . . . . . . . . . . . . . . 49

5.2 .5 Key Selection . . . . . . . . . . . . . . . . . . . 49

5.2.6 Customized Encryption . . . . . . . . . . . . . . . 50

5.2.7 Client-Server Environment . . . . . . . . . . . . . . 54

6 Conclusion and Future Work $\quad 55$

6.1 Limitations . . . . . . . . . . . . . . . . . . 55

6.2 Conclusion . . . . . . . . . . . . . . . . 56

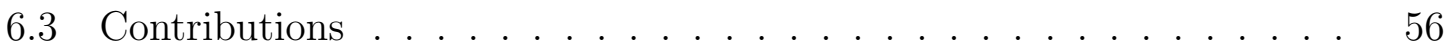

$\begin{array}{ll}\text { References } & 58\end{array}$ 


\section{List of Tables}

3.1 Standard Polynomials Table for $\mathrm{m}=2-128[24] \ldots$. . . . . . . . . . . 20

3.2 Multiplicative inverse table in $\mathrm{GF}\left(2^{8}\right)$ for $x^{8}+x^{4}+x^{3}+x+1 \quad \ldots . . . \quad$. . 21

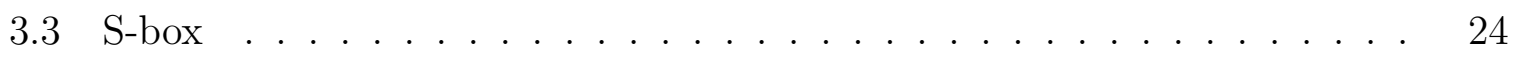

3.4 Inverse S-box . . . . . . . . . . . . . . . . . . 30

4.1 Irreducible Polynomial Equations . . . . . . . . . . . . . . . . . . 32

4.2 S-box and Inverse S-box for $x^{8}+x^{4}+x^{3}+x^{2}+1 \ldots \ldots$. . . . . 32

4.3 S-box and Inverse S-box for $x^{8}+x^{5}+x^{3}+x+1 \ldots \ldots$. . . . . . 33

4.4 S-box and Inverse S-box for $x^{8}+x^{5}+x^{3}+x^{2}+1 \ldots \ldots 33$

4.5 S-box and Inverse S-box for $x^{8}+x^{6}+x^{3}+x^{2}+1 \quad \ldots . \ldots . . . . \quad 33$

4.6 S-box and Inverse S-box for $x^{8}+x^{6}+x^{4}+x^{3}+x^{2}+x+1 \ldots . . . \quad 34$

4.7 S-box and Inverse S-box for $x^{8}+x^{6}+x^{5}+x+1 \ldots \ldots$. . . . . . 34

4.8 S-box and Inverse S-box for $x^{8}+x^{6}+x^{5}+x^{2}+1 \ldots \ldots$. . . . . . 34

4.9 S-box and Inverse S-box for $x^{8}+x^{6}+x^{5}+x^{3}+1 \quad \ldots \ldots \ldots$

4.10 S-box and Inverse S-box for $x^{8}+x^{6}+x^{5}+x^{4}+1 \quad \ldots . \ldots . . . . \quad 35$

4.11 S-box and Inverse S-box for $x^{8}+x^{7}+x^{2}+x+1 \ldots \ldots$

4.12 S-box and Inverse S-box for $x^{8}+x^{7}+x^{3}+x^{2}+1 \ldots \ldots$

4.13 S-box and Inverse S-box for $x^{8}+x^{7}+x^{5}+x^{3}+1 \ldots \ldots$

4.14 S-box and Inverse S-box for $x^{8}+x^{7}+x^{6}+x+1 \ldots \ldots$

4.15 S-box and Inverse S-box for $x^{8}+x^{7}+x^{6}+x^{3}+x^{2}+x+1 \ldots 37$

4.16 S-box and Inverse S-box for $x^{8}+x^{7}+x^{6}+x^{5}+x^{2}+x+1 \ldots 37$

4.17 S-box and Inverse S-box for $x^{8}+x^{7}+x^{6}+x^{5}+x^{4}+x^{2}+1 \ldots 37$ 
5.1 Security Services offered by the Protocol . . . . . . . . . . . . . . 46 


\section{List of Figures}

2-1 Caesar Cipher with a shift of 2 to the right $[1] \ldots \ldots$

2-2 A three-wheel Enigma machine $[1] \ldots \ldots$. . . . . . . . . . . . . . 6

2-3 Communication over an insecure channel . . . . . . . . . . . . . . . 7

2-4 Symmetric-key cryptosystem . . . . . . . . . . . . . . . . 8

2-5 Principles of encrypting $\mathrm{n}$ bits with stream and block ciphers . . . . . . . 9

2-6 Basic AES block . . . . . . . . . . . . . . . . . . 9

2-7 Analogy for Symmetric Encryption . . . . . . . . . . . . . . . . 10

2-8 Analogy for Asymmetric Encryption . . . . . . . . . . . . . . 11

2-9 Protocol for Public-Key Encryption . . . . . . . . . . . . . . . . . 12

2-10 Example of RSA Encryption . . . . . . . . . . . . . . . . . . 14

2-11 Diffie-Hellman Key Exchange . . . . . . . . . . . . . . . . . 15

2-12 Principle of Digital Signatures . . . . . . . . . . . . . . 16

3-1 Structure of AES . . . . . . . . . . . . . . . . . . . 19

3-2 AES process flowchart . . . . . . . . . . . . . . . 23

3-3 S-box construction . . . . . . . . . . . . . . . . . . . . 23

3-4 Affine Mapping . . . . . . . . . . . . . . . . . . . . . . . . . . . . . . . . 24

3-5 Input and Output matrices . . . . . . . . . . . . . . 25

3-6 Mixcolumn Layer . . . . . . . . . . . . . . . . . . . . 26

3-7 AES key scedule for 128-bit key size [24] . . . . . . . . . . . . . . . . 27

3-8 AES decryption block diagram . . . . . . . . . . . . . . . 28

3-9 Inverse Mixcolumn Layer . . . . . . . . . . . . . . . . . . . . . . . 29 
3-10 Matrices in Inverse Shiftrows _ . . . . . . . . . . . . . . . . . . 29

3-11 Inverse Affine Mapping . . . . . . . . . . . . . . . . . . . . . . . . . . . . 30

4-1 Gate Function Block . . . . . . . . . . . . . . . . . . 39

4-2 Protocol for Key Establishment . . . . . . . . . . . . . . . . . . . . . . 40

4-3 Communication protocol between devices . . . . . . . . . . . . . . . . . . 41

$4-4$ Centralized Server . . . . . . . . . . . . . . . . . . . 43

5-1 Attack Scenario . . . . . . . . . . . . . . . . . . . . 45

$5-2 \quad$ Keys Generation . . . . . . . . . . . . . . . . . 47

5-3 Hash Generation . . . . . . . . . . . . . . . . . . . . . 48

5-4 Encryption and Decryption of Hash . . . . . . . . . . . . . . . . . 48

$5-5$ Gate Function . . . . . . . . . . . . . . . . . . . . . . . . 49

$5-6 \quad$ Key Selection . . . . . . . . . . . . . . . . . . . 50

5-7 Importing selected S-box and Inverse S-box _ . . . . . . . . . . . . 51

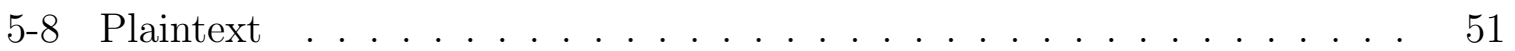

5-9 Customized Encryption _. . . . . . . . . . . . . . 51

5-10 Ciphertext . . . . . . . . . . . . . . . . . 52

$5-11$ Decryption . . . . . . . . . . . . . . . . 52

$5-12$ Decrypted Text . . . . . . . . . . . . . . . . . 52

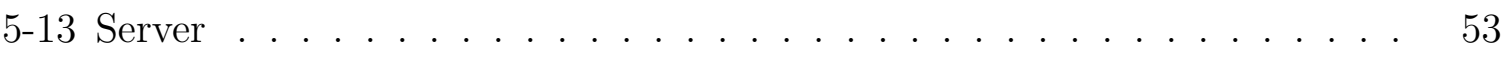

$5-14$ Client . . . . . . . . . . . . . . . . . . 53 


\title{
List of Abbreviations
}

\author{
AES $\ldots \ldots \ldots \ldots \ldots \ldots$ Advanced Encryption Standard \\ DES $\ldots \ldots \ldots \ldots \ldots \ldots$ Data Encryption Standard \\ DHKE $\ldots . . . \ldots \ldots \ldots . .$. Diffie Hellman Key Exchange \\ EEA $\ldots \ldots \ldots \ldots \ldots \ldots$. Extended Euclidean Algorithm \\ FPGA $\ldots \ldots \ldots \ldots \ldots$. Field Programmable Gate Arrays \\ GCD $\ldots \ldots \ldots \ldots \ldots \ldots$ Greatest Common Divisor \\ GF $\ldots \ldots \ldots \ldots \ldots \ldots$ Galois Fields \\ IoT $\ldots \ldots \ldots \ldots \ldots \ldots$ Internet of Things \\ LAN $\ldots \ldots \ldots \ldots \ldots \ldots$........... Local Area Network \\ MD-5 .............. Message Digest-5 \\ MIME $\ldots \ldots \ldots \ldots \ldots \ldots$ Multipurpose Internet Mail Extensions \\ MITM $\ldots \ldots \ldots \ldots \ldots \ldots$ Man-In-the-Middle \\ PGP $\ldots \ldots \ldots \ldots \ldots \ldots$. Pretty Good Privacy \\ RNG $\ldots \ldots \ldots \ldots \ldots$. Random Number Generator \\ RSA $\ldots \ldots \ldots \ldots \ldots \ldots$ Ron Rivest, Adi Shamir, and Leonard Adleman \\ SHA $\ldots \ldots \ldots \ldots \ldots$. Secure Hash Algorithm \\ SSH $\ldots \ldots \ldots \ldots \ldots \ldots$. Secure Socket Shell \\ SSL $\ldots \ldots \ldots \ldots \ldots \ldots$. Secure Socket Layer \\ TLS $\ldots \ldots \ldots \ldots \ldots \ldots$ Transport Layer Security
}




\section{Chapter 1}

\section{Introduction}

\subsection{Problem Statement}

In the current world, encryption plays a significant role in securing relevant information from eavesdroppers, attackers, and unauthorized users. The applications of such protocols fall under numerous categories ranging from internet banking to internet of things. With IoT trending in the market, it is more important to ensure the authentication of the connected devices. We come across a lot of attacks where an unknown user tries to interrupt communication between two authorized devices. In most of the cases, the devices get connected through a handshake protocol where they agree on a common encryption algorithm to be used and thereby establish the secure communication medium and share the necessary keys.

If the malicious user has access to shared keys between devices, there could be chances of determining the encryption algorithm by launching many attacks. Thus, it is necessary to develop secure authentication schemes where the attacker can't imitate someone's identity and get access to the keys. In addition to it, it is important to build strong and complex encryption protocols to prevent the attacker from knowing the algorithm used. 


\subsection{Proposed Research Approach}

AES is the most widely used encryption algorithm in different applications [7]. One of its main implementations is in the SSL and TLS protocols [20]. The computational speed of the algorithm makes it more efficient. In the process of developing a new algorithm, it is important to keep in mind the algorithm has to be as productive as the existing ones, if not better. Therefore, a customized encryption technique which is a variation of AES is introduced. The AES works on a standard polynomial equation which contributes towards various building layers of the algorithm [24]. The proposed algorithm uses all the sixteen irreducible polynomials, to implement encryption. The algorithm devises a new approach towards key establishment between the communicating devices. The implementation of the algorithm in Python is illustrated in the thesis.

An authentication protocol along with the communication protocol using a centralized server is also presented in this thesis. The devices request the centralized server for access to communicate with the intended parties. The centralized server acts as trusted third party and verifies the authenticity of the connecting devices. The attacks that can be controlled using the above authentication scheme are described.

\subsection{Thesis Organization}

The rest of the thesis is organized as follows:

Chapter 2 provides a literature review on the basic introduction to cryptography. The classification of encryption protocols and their applications are described.

Chapter 3 presents the detailed explanation of the AES algorithm, which is needed to understand the proposed approach.

Chapter 4 introduces the methodology and protocols of the customized encryption approach. 
Chapter 5 presents the security features offered by the protocol and its implementations in Python.

Chapter 6 includes limitations, conclusion and future work. 


\section{Chapter 2}

\section{Literature}

\section{$2.1 \quad$ Introduction}

Cryptography is an art of hiding confidential information from a third party by implementing keys which are only known by the communicating parties [14]. Since this is a secured form of transmitting information, it is used in bank card payments, electronic building, electronic commerce, etc. It is essential that modern connected organizations have an idea of cryptography and its applications. Cryptography can help in securing online servers with the right implementation. Incorrect application of cryptography can result in disclosure of sensitive information and give the user a false sense of security.

\subsection{Background and Motivation}

Cryptography has been in implementation since communication originated. The cryptic messages found in Egyptian and Mesopotamian ruins are considered as a form of cryptography. Caesar Cipher is one of the well-known examples of early cryptographic techniques. This cipher is a technique of substituting letters in the alphabet with letters further in the alphabet. This works cyclically, $\mathrm{C}$ is replaced by $\mathrm{D}$ and $\mathrm{D}$ is replaced by $\mathrm{E}$ and so on. This cipher is also known as a substitution cipher 
[1]. The example of a Caesar Cipher is shown in Fig. 2-1. The historical Ciphers rely on a principle called security by obscurity, which means the safety of the cipher is reliant on the algorithm being a secret.

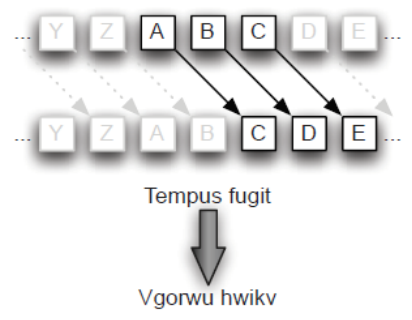

Figure 2-1: Caesar Cipher with a shift of 2 to the right [1]

As we all know, Cryptography played a significant role in World War II. The Germans and its allies invested a lot of time and revenue on building cryptosystems. They were a combination of mechanical and electronic systems. Enigma is a wellknown cryptosystem. Fig. 2-2 shows an example of the Enigma system. It uses a symmetric key algorithm (i.e., a single key used to encrypt and decrypt the messages). The enigma is electromechanical and uses rotating wheels (rotors) for operation. A daily code and wire settings along with the initial settings are used to determine the key being used. Enigma was used by all the branches of the German army.

Information Security made some significant leaps with the advancement of modern digital computers. Algorithms which were considered to be unbreakable are now deemed to be trivial to break [10]. Asymmetric cryptography is the most important modern cryptographic invention. Asymmetric Cryptography supports sharing of information publically (having a public key) which is used to encrypt the data and transmit. The transmitted data can be decrypted only by the corresponding secret key (private key). 


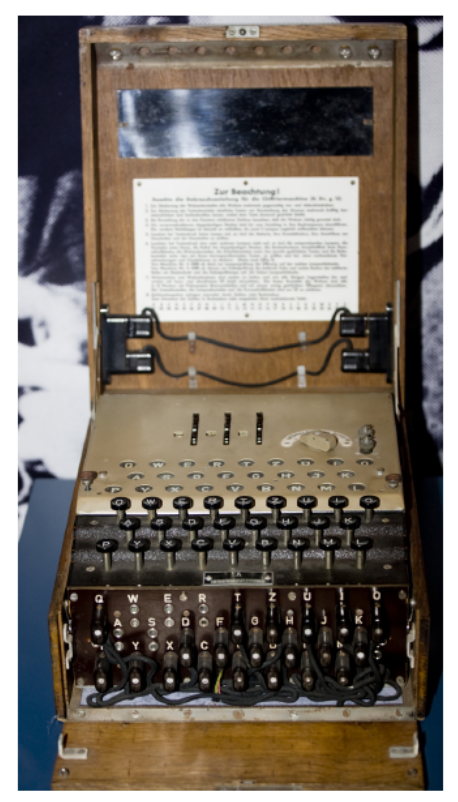

Figure 2-2: A three-wheel Enigma machine [1]

\subsection{Classifications}

There are three main branches of Cryptography: Symmetric systems, Asymmetric systems, and Cryptographic protocols. Secure internet communication can be realized with Symmetric and Asymmetric algorithms. Cryptographic protocols deal with the application of cryptographic algorithms [8]. Every web browser uses the Transport Layer Security (TLS) scheme, which is an example of crypto protocols.

Symmetric and Asymmetric (public) key systems are the basic types of cryptography in common use. Both symmetric and asymmetric algorithms are used together in the majority of applications in practical systems. These algorithms are used together since both algorithms have their particular strengths and weaknesses. This is often known as hybrid scheme [21]. The strength of the cryptosystem is proportional to the length of the key. Having a longer key leads to a full utilization of the key space and eliminating the inherent weakness in the algorithm. 


\subsubsection{Symmetric Cryptography}

The general perception of symmetric algorithms is that the two communicating parties have encryption and decryption method for which they have a secret key. Until 1976, the cryptographic techniques used were exclusively based on symmetric methods. Applications like data encryption and integrity checking of messages still use symmetric ciphers. Symmetric cryptography is also known as a secret-key algorithm. Let us consider an easy example for a better introduction to symmetric cryptography: Two users Emma and David want to communicate through an insecure channel as illustrated in the Fig. 2-3

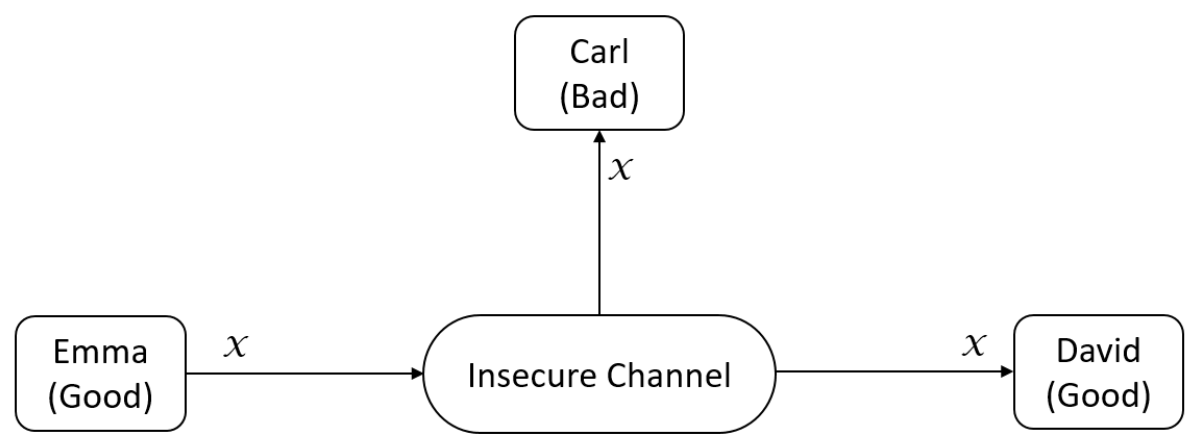

Figure 2-3: Communication over an insecure channel

Channel is a general term used for the communication link. This channel can be the air in wireless communication or wireless LAN communication or any of the communication media. Now, the problem starts with Carl, who is a malicious user. Let's assume Carl got access to the channel, by hacking into the internet router or by interpreting the radio signals of the Wi-Fi communication. This kind of unauthorized listening is known as eavesdropping. There will be situations where Emma and David will want to communicate without Carl listening. For instance, if Emma and David want to transmit documents containing business strategies, this information should not get into the hands of their competitors. 
In this case, symmetric cryptography presents a practical solution: Emma encrypts her message A using a symmetric algorithm, which will result in a ciphertext B. David receives the ciphertext and decrypts the message. The main advantage of this is the encrypted message will be displayed as random bits to the user Carl. The variables $\mathrm{A}, \mathrm{B}$, and $\mathrm{K}$ in Fig. 2-4 are important in cryptography and have unique names: $\mathrm{A}$ is called plaintext or cleartext $\mathrm{B}$ is called ciphertext $\mathrm{K}$ is called key

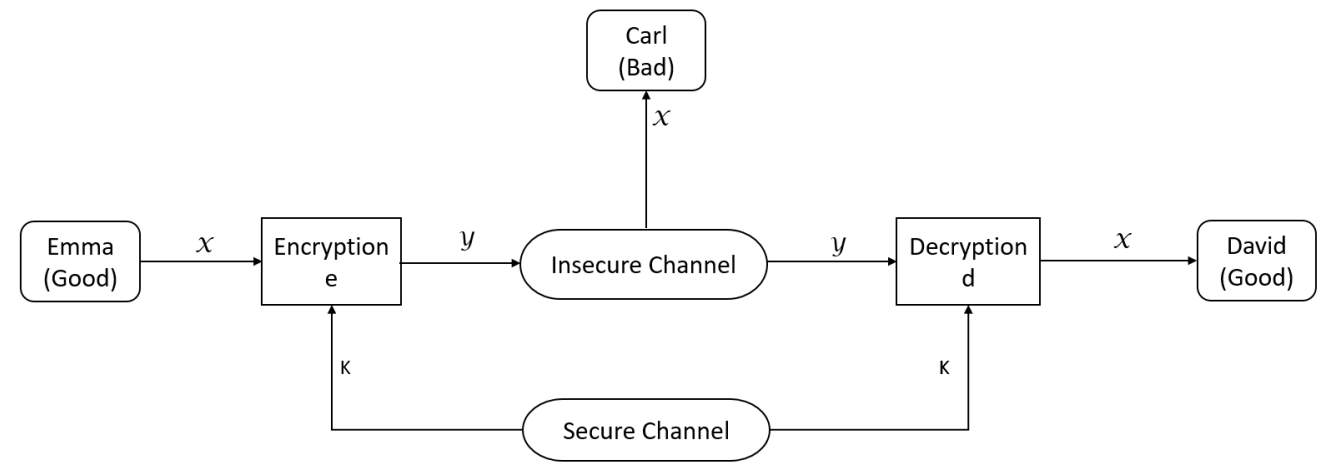

Figure 2-4: Symmetric-key cryptosystem

Symmetric cryptography can be split into block ciphers and stream ciphers. Fig. 2-5 depicts the operational differences of a block cipher and stream cipher. Bits are encrypted individually in stream ciphers [26]. A bit from a key stream is added to a plain text to achieve this. An entire block of plaintext is encrypted with the same key in block ciphers. The encryption of the plaintexts in any given block is related to the encryption of another plaintext of the same block. Practically, the majority of block ciphers have a block length of 128 bits(16 bytes) like Advanced Encryption Standard (AES), or a length of 64 bits (8 bytes) like Data Encryption Standard (DES) [3] or Triple DES (3DES) algorithm. AES and DES are examples of the symmetric algorithm. But, these are some of the many symmetric algorithms. Hundreds of algorithms have been proposed over the years. Even though some of these proposed algorithms were deemed insecure, many other cryptographically secure ones exist in the market. 

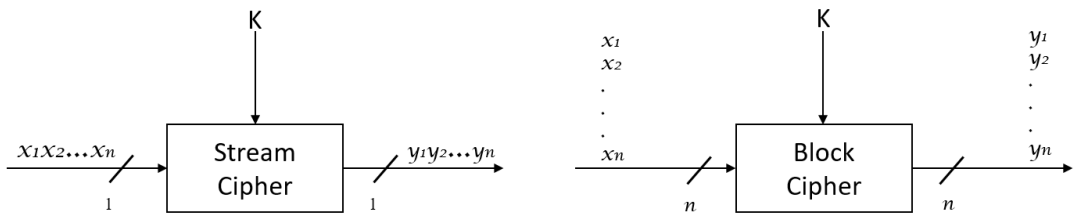

Figure 2-5: Principles of encrypting $\mathrm{n}$ bits with stream and block ciphers

Advanced Encryption Standard (AES) is the prominent choice of algorithm for encryption which will be discussed further on. The AES cipher is similar to block cipher Rijndael. Rijndael with a block length of 128 bits is known as the AES algorithm. $[24]$

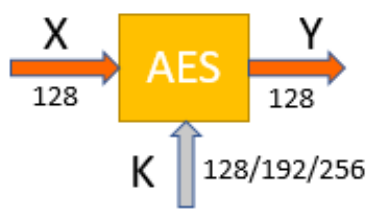

Figure 2-6: Basic AES block

AES has survived decades of brute force attacks with its three high key lengths of 128, 192 and 256 bits shown in Fig. 2-6. Any analytical attacks with a reasonable chance of success are still unknown. AES was a result of an open competition. There were four other potential strong finalist algorithms, Mars, RC6, Serpent, and Twofish. These were cryptographically strong and quite fast, particularly in software. Mars, Serpent, and Twofish are royalty-free. Triple DES [18] often denoted as 3DES is an alternate to AES or the AES finalist algorithms. Three subsequent DES encryption with different keys are present in 3DES. 3DES does not support software as efficiently as it supports hardware. Financial applications and protection of biometric information in electronic passports use 3DES. The DES is made much more resistant against exhaustive key searches by this simple modification. 


\subsubsection{Asymmetric Cryptography}

Public-key algorithms vary from symmetric key algorithms like AES or DES. Public-key algorithms are usually based on number-theoretic functions, which are different from symmetric ciphers. The aim is not to have complex mathematical description between input and output. Let us recall the basics of encryption schemes to understand the principle of asymmetric cryptography.

Fig. 2-7 shows a simple analogy for symmetric cryptography. Let us assume a vault, with a very secure lock. Only Emma and David have the key to the lock. The process of encrypting a message is viewed as putting a message in that safe. To read that message (i.e., decrypt the message), David uses his key to open the vault.

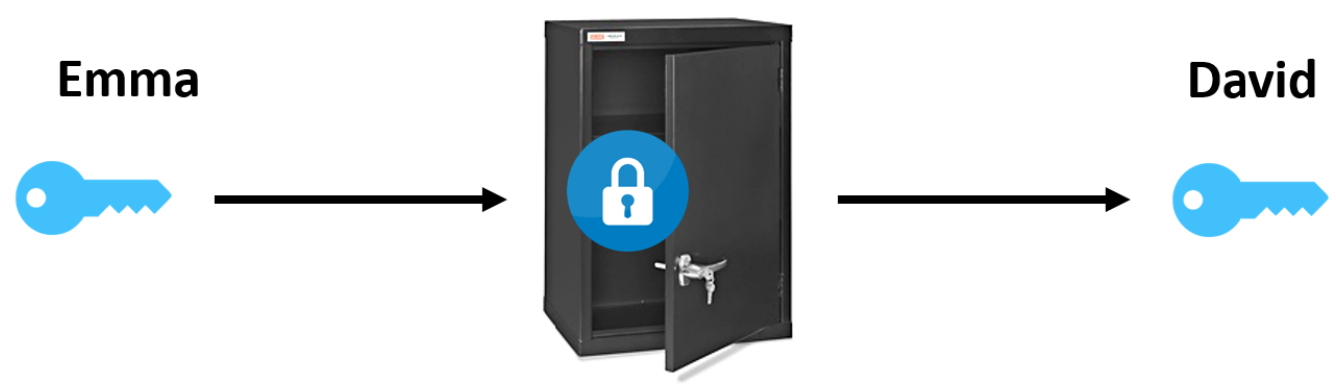

Figure 2-7: Analogy for Symmetric Encryption

Key distribution problems are some of the several shortcomings linked with symmetrickey schemes [23]. The key should be shared between Emma and David using a secure medium (channel). Sending the key can not be done if the channel is not secure. Even after solving the key distribution problem, we must be ready to deal with a huge number of keys. If each user has a unique key, there will be $\mathrm{n}$ number of users with $n^{*}(n-1) / 2$ key pairs. Every user must store (n-1) keys securely. Even for a midsize network, say 2000 personnel, we require more than 4 million key pairs that need to be generated and transmitted using secured channels.

Emma and David have the same abilities because they have the same keys. If 


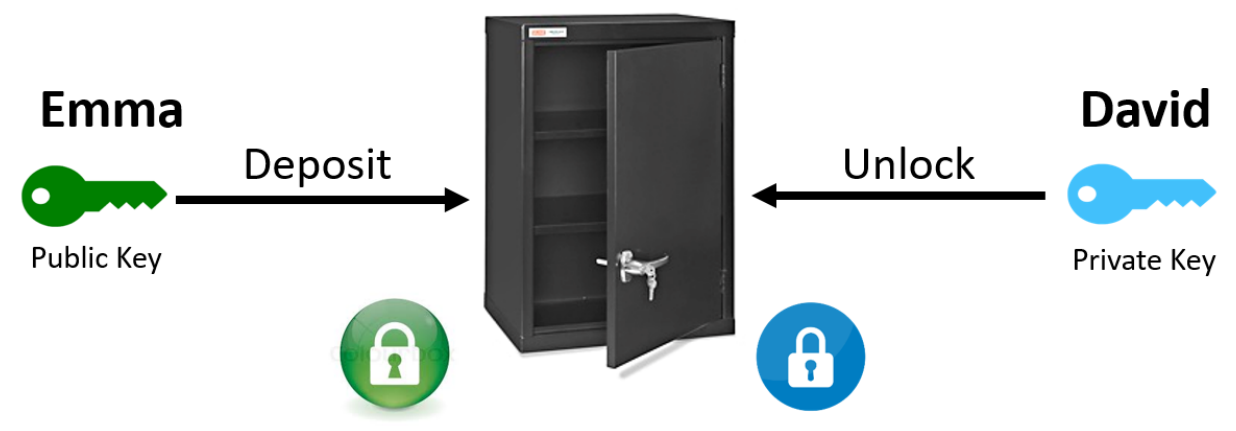

Figure 2-8: Analogy for Asymmetric Encryption

we intend to oppose any cheating from Emma or David instead of Carl, symmetric cryptography is of little use. Let us assume that Emma has ordered something online. If she changes her mind after a while, she can claim that David has falsely generated the purchase order. The prevention of this is called non-repudiation which will be explained in further chapters. These faults can be prevented by using asymmetric cryptography.

To overcome these deficiencies, Diffie, Hellman, and Merkel proposed a revolutionary idea: The secrecy of encryptor's key (Emma in our case) is not very important. The essential part is that the receiver (David in our case) can decrypt the message using only a secret key. To accomplish this, David publishes a public key, which can be utilized by anyone who wants to send him a message. This public key has a matching secret key which can be used to decrypt. Let us assume that David has a key K. K has two parts $K_{p u b}$ (public key), $K_{p r}$ (private key). A simple illustration of this is shown in Fig. 2-8. The more general example of this is a standard old mailbox; anyone can put a letter in it (encrypt), but only an individual with the private (secret) key to the mailbox can retrieve the letters (decrypt).

A general public-key encryption (assuming we have cryptosystems with these functionalities) looks as shown in Fig. 2-9. Public-key algorithms have three major families which are of practical relevance; these are RSA, Discrete logarithm, and Elliptic 


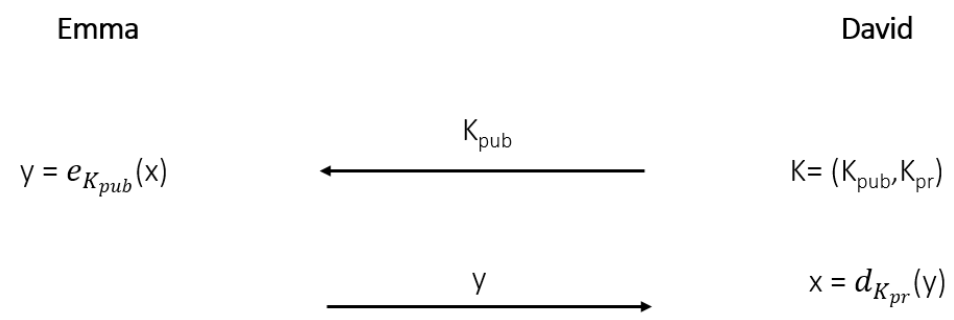

Figure 2-9: Protocol for Public-Key Encryption

curve.

The Rivest-Shamir-Adleman algorithm (RSA) crypto scheme, is currently one of the more prominently used asymmetric cryptography schemes, although elliptic curves and discrete logarithm schemes are gaining prominence. RSA has many applications, but in practice, it is used for encryption of small pieces of data and digital signatures. Its primary purpose is to transfer keys. The main reason for RSA to become the more widely used asymmetric algorithm is its attributes, such as confidentiality, integrity, authenticity, and non-reputability of storage data and also electronic communications. Few protocols such as OpenPGP, SSH, S/MIME, and SSL/TLS are dependent on RSA for encryption and digital signature functions. Further, it is used in software applications like browsers, which need a secure connection over an insecure network such as the Internet or to validate a digital signature. RSA signature verification is a highly performed operation in IT.

RSA is secure because of the difficulty in factoring large integers that are a multiple of two prime numbers [5]. Finding the product of two prime numbers is easy, but determining the original prime numbers from the total factoring is considered infeasible even with today's supercomputers. The generation of public and private keys is the most complex part of RSA cryptography. The following protocol displays the steps in RSA key generation:

1. Choose large prime numbers $\mathrm{p}$ and $\mathrm{q}$. 
2. Calculate $\mathrm{n}=\mathrm{p}^{*} \mathrm{q}$.

3. Caluclate $\phi(n)=(p-1) *(q-1)$.

4. Choose the exponent $\mathrm{e} \in\{1,2,,(n)-1\}$ such that $\operatorname{gcd}(\mathrm{e}, \phi(\mathrm{n}))=1$.

5. Compute private key $\mathrm{d}$ such that $\mathrm{d}^{*} \mathrm{e}=1 \bmod \phi(\mathrm{n})$.

Where,

Public key $\left(K_{p u b}\right)=(\mathrm{n}, \mathrm{e})$

Private key $\left(K_{p r}\right)=\mathrm{d}$

The first step describes the generation of two large prime numbers (let's say $\mathrm{p}$ and q), using Robin-Miller primality test algorithm. By multiplying p and q, the modulus $\mathrm{n}$ is calculated. This number is utilized by both private and public keys and generates a link between them. Its length is usually expressed in bits and is called the key length. The public key consists of modulus n, and a public exponent, e, which is commonly set at 65537 , since it is a prime number which is not too large. The public exponent e doesn't have to be a secretly selected prime number as a public key since it is shared openly. The private key has the modulus 'n' and a private exponent 'd' which is calculated using the Extended Euclidean algorithm to determine the [1] multiplicative inverse with respect to the quotient of n. An example of RSA encryption is shown in Fig. 2-10

The sizes of RSA are usually 512, 1024, 2048-bits. But, RSA encryption is not meant to replace symmetric ciphers because it is much slower than ciphers like AES. RSA is slower than AES because of the computations involved in performing publickey algorithms [27]. Hence, the primary use of encryption process is to reliably exchange a key for a symmetric cipher (key transport). Usually, in practice, RSA is used along with a symmetric cipher like AES (generally known as Diffie-Hellman Key Exchange), where the bulk data encryption is done by the symmetric cipher. 
Emma

message is, $x=4$

$y=x^{e}=4^{3}=31 \bmod 33$
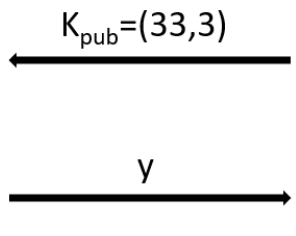

David

- Chooses $p=3, q=11$

- $\mathrm{n}=33$

- $\varnothing(n)=(3-1) \cdot(11-1)$

20

- Choose e $=3$

- $\mathrm{d}=\mathrm{e}^{-1}=7 \bmod 20$

To decrypt $x$, $\mathrm{x}=\mathrm{y}^{\mathrm{d}}=31^{7}=4 \bmod 33$

Figure 2-10: Example of RSA Encryption

\subsection{Diffie Hellman Key Exchange (DHKE)}

The Diffie-Hellman Key Exchange (DHKE), proposed by Whitfield Diffie and Martin Hellman in 1975, was the first asymmetric scheme published in open literature. It provides a reasonable solution to the key distribution problem. It allows two users to get a common secret key while communicating through an insecure channel [11]. This fundamental key agreement is performed in several open and modern cryptographic protocols like Internet Protocol Security (IPSec) etc.

DHKE has two protocols, main protocol, which implements the computations and a set-up protocol, which implements the actual key exchange. The set-up protocol consists of the following steps shown below:

1. Choose a large prime number $\mathrm{p}$.

2. Choose an integer $\alpha \in\{2,3,, p-2\}$.

3. Declare $\mathrm{p}$ and $\alpha$

The two values $\mathrm{p}$ and $\alpha$ are referred to as domain parameters. If Emma and David both know the public parameters $\mathrm{p}$ and $\alpha$ computed in the set-up phase, they can create a common secret key $\mathrm{K}$ with the following key-exchange shown in Fig. 2-11. 


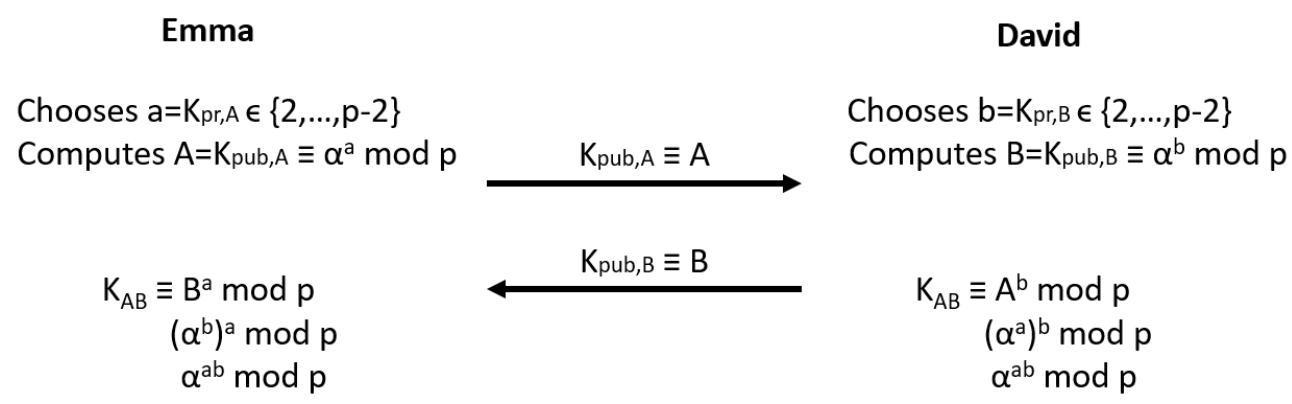

Figure 2-11: Diffie-Hellman Key Exchange

Emma and David both derive the same session key. This key can then be used to establish a safe communication between them. For example, by utilizing $K_{a b}$ as key for symmetric algorithms like AES or 3DES. For example, if AES is chosen as the algorithm to encrypt data since the session key is of 1024 bits, the 128 Most Significant Bits (MSB) are selected as the 128 bit key to AES. For the Diffie-Hellman protocol, the prime p has to be at least 1024 bits long. Thus, this protocol results in reliable cryptosystems.

\subsection{Digital Signatures}

The Cryptosystems we have studied so far had two primary aims: either to encrypt data or to establish a shared key. That alone does not give us the necessary tools to satisfy any security requirements other than encryption and key exchange. The property of proving that the message has been generated by a particular person is also very important outside the digital domain to reduce the certain risk of attacks. In the analog world, this can be achieved by handwritten signatures on paper. For example, if we sign a document, the receiver can prove to the judge that we signed the document. In the digital world, the individual who creates the digital message must be capable of generating a valid signature [25].

For us to achieve this with cryptographic primitives, public-key cryptography 
must be performed. The general idea is that the person who signs the message uses a private key and the receiver uses the matching public key. Digital signatures offer non-repudiation [31], i.e. person who sends the message cannot deny the creation of the message. The central principle of the digital signature scheme is shown in Fig. 2-12. Emma computes the message and signs it using her private key. David decrypts the message and verifies the signature using the public key shared by Emma.

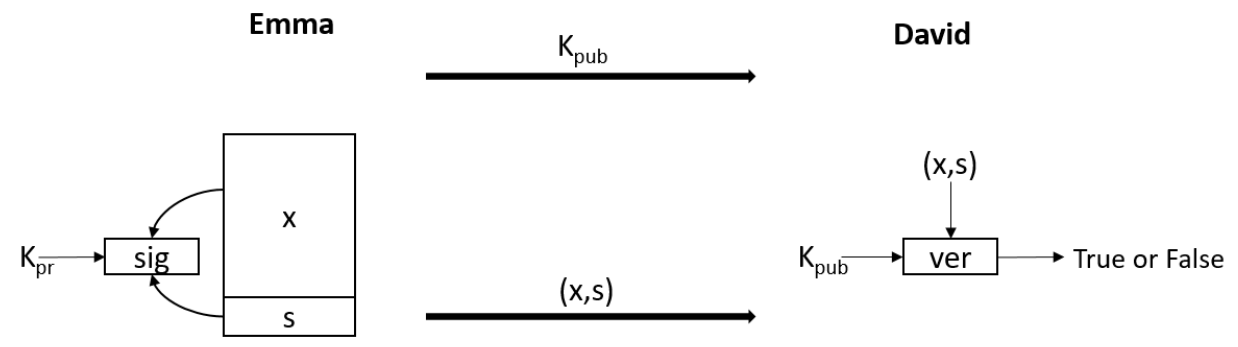

Figure 2-12: Principle of Digital Signatures

Hash functions are an important part of digital signature schemes as well. They compute a digest for a given message, which is short and a fixed-length string. The hash value or the message digest is regarded as a fingerprint of the message. Hence, every input generates one hash output and is one way-ed i.e. it is difficult to derive the input using output. SHA-1, SHA-2, MD-5 algorithms are popular examples of hash functions [13]. As described in the earlier chapters, RSA signature scheme is based on the RSA encryption. The RSA signature scheme is regarded as the most widely used digital signature scheme in practice. To utilize RSA to sign a message digitally, Emma would create a message digest of her message to David, encrypts the hash value with the RSA private key and adds it to the message. David can then verify that message has been sent by Emma and has not been tampered with by decrypting the hash value using her public key. If this matches with the hash value of the original message, then only Emma could have seen it (authentication and nonrepudiation) and the message is exactly as she wrote it (integrity). Emma can also 
encrypt her message with David's RSA public key (confidentiality) before sending it to David.

\subsection{Summary}

As cryptography is a vast topic, only the introductory topics are sufficient to understand the proposed encryption algorithm. Even though there are many encryption techniques which provide security, there is always scope to develop and implement new protocols. One such protocol which is a variation of Advanced Encryption Standard (AES) algorithm with a different authentication scheme is implemented in the thesis. To understand the customized protocol, detailed understanding of AES is needed and is explained in the following chapter. 


\section{Chapter 3}

\section{Standard Encryption Approach}

\subsection{AES Encryption}

As described in the introduction, AES is a 128-bit block cipher with key sizes of 128, 192 and 256 bits. Fig. 3-1 describes the structure of AES. It comprises of three layers, Key Addition layer, Byte Substitution Layer and Diffusion Layers (Shiftrow and Mixcolumn) respectively. Each layer manipulates 128 bits of the data. Before going through the process of how AES converts the plain text into cipher text, one has to know the standard properties exhibited by the AES fields, which are used in every layer of the algorithm. AES implementations are not limited to software applications. It is also used in hardware implementations such as FPGA's [15]. 


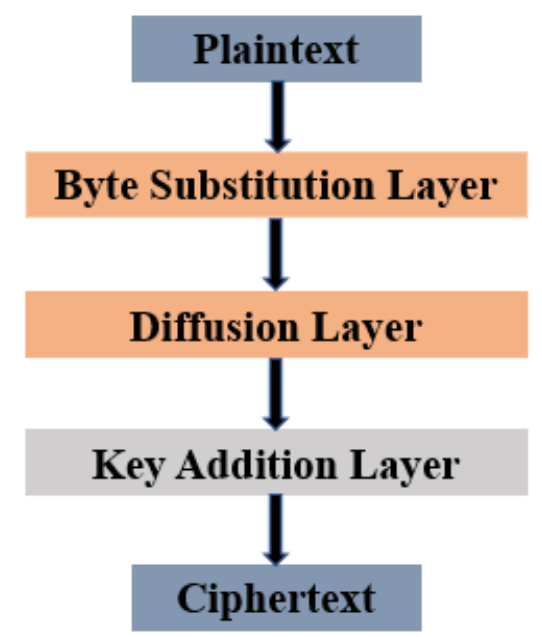

Figure 3-1: Structure of AES

\subsection{Galois Fields}

Extension fields, also known as Galois Fields consist of $\operatorname{GF}\left(2^{m}\right)$ elements where $\mathrm{m}>1$. A Galois field with a set of $256\left(\mathrm{GF}\left(2^{8}\right)\right)$ elements is called the AES field. All the elements are represented as polynomial equations with their 8-bit binary representations filling the coefficients of the equations. For instance, the element 52 (00110100) can be represented as $x^{5}+x^{4}+x^{2}$. The elements form a group in which we can add, subtract, multiply, and all the operations are performed in modulo arithmetic. Addition operation is used by the Key addition layer of the AES and is achieved by performing standard polynomial addition. Coefficients with equal powers of $\mathrm{x}$ are added or subtracted depending on the operation. Moreover, the addition modulo 2 performed in the AES fields is equal to the bit wise XOR operation. Therefore, addition and subtraction are the same in these fields. 
Table 3.1: Standard Polynomials Table for $\mathrm{m}=2-128$ [24]

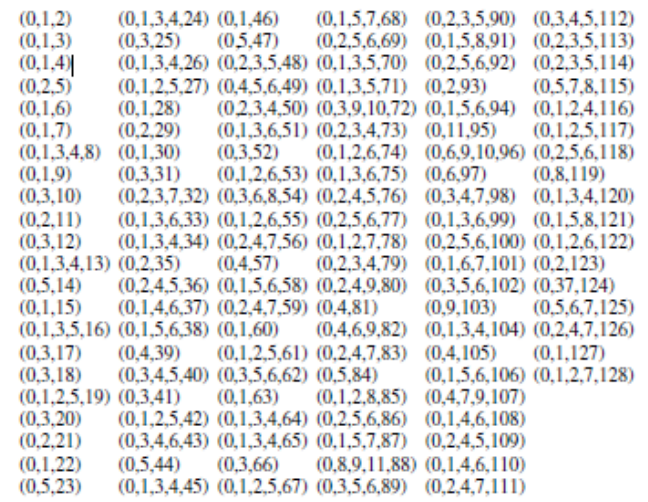

\subsubsection{Multiplication in Galois Fields}

Multiplication in $\operatorname{GF}\left(2^{8}\right)$ [28] is one of the main operations of the Mix Column transformation layer in AES. The two elements (represented by their polynomials) of a finite field are multiplied using the standard polynomial multiplication rule, i.e., the sum or product of any two elements from the field must be an element within the field. In general, the product polynomial will have a degree higher than $\mathrm{m}$ 1 which must be reduced. The basic idea is similar to multiplying two integers, dividing the result by a prime, and considering only the remainder as a solution. Likewise in the extension fields, the product of the multiplication is divided by a certain polynomial, and only the remainder of the polynomial division is considered as the result. Modulo reduction is performed with the help of irreducible polynomials. Irreducible polynomials are comparable to prime numbers, i.e., their only factors are 1 and the polynomial itself. For every value of $m$ in $\operatorname{GF}\left(2^{m}\right)$, there exists a few irreducible polynomials $\mathrm{P}(\mathrm{x})$ of degree $\mathrm{m}$ with coefficients from $\mathrm{GF}(2)$ (a prime field which consists of only two elements 0 and 1). However, an important point to be noted is that not all polynomials are irreducible. For example, the polynomial $x^{4}$ $+x^{3}+x^{2}+x$ is reducible since $x^{4}+x^{3}+x^{2}+x=\left(x^{3}+x\right)(x+1)$ and hence cannot be used to construct the extension field $\mathrm{GF}\left(2^{4}\right)$. Many primitive or irreducible 
Table 3.2: Multiplicative inverse table in $\mathrm{GF}\left(2^{8}\right)$ for $x^{8}+x^{4}+x^{3}+x+1$

\begin{tabular}{|c|c|c|c|c|c|c|c|c|c|c|c|c|c|c|c|c|}
\hline & 0 & 1 & 2 & & & 3 & 6 & ' & 8 & & A & B & C & D & $\mathrm{E}$ & \\
\hline & 00 & 01 & $8 D$ & F6 & CB & 52 & 7B & D1 & E8 & $4 \mathrm{~F}$ & 29 & Co & BO & E1 & E5 & 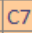 \\
\hline & 74 & B & A & & 99 & $2 \mathrm{~B}$ & 60 & $5 \mathrm{~F}$ & 58 & $3 F$ & D & CC & & 40 & $\mathrm{EE}$ & \\
\hline & $3 \mathrm{~A}$ & 6 & A & $L$ & 55 & $4 \mathrm{D}$ & A8 & C9 & $\mathrm{C} 1$ & $\mathrm{OB}$ & 98 & 15 & 30 & 44 & 2 & \\
\hline & $2 C$ & & 2 & & F3 & 39 & 66 & 42 & F2 & 35 & 20 & $6 \mathrm{~F}$ & 77 & BB & 59 & \\
\hline & 1D & F & 37 & 67 & $2 D$ & 31 & F5 & 69 & A7 & 64 & $\mathrm{AB}$ & 13 & 54 & 25 & E9 & \\
\hline & ED & $5 C$ & 05 & CA & $4 C$ & 24 & 87 & BF & 18 & $3 \mathrm{E}$ & 22 & Fo & 51 & EC & 61 & 17 \\
\hline & 16 & $5 \mathrm{E}$ & AF & D3 & 49 & A6 & 36 & 43 & $\mathrm{~F} 4$ & 47 & 91 & DF & 33 & 93 & 21 & $3 B$ \\
\hline & 79 & B7 & 97 & 85 & 10 & B5 & BA & $3 C$ & B6 & 70 & D0 & 06 & A1 & FA & 81 & 82 \\
\hline & 83 & 7 & $7 F$ & 80 & 96 & 73 & $\mathrm{BE}$ & 56 & 9B & $9 \mathrm{E}$ & 5 & D9 & F7 & 02 & B9 & A4 \\
\hline & $\mathrm{DE}$ & $6 \mathrm{~A}$ & 32 & $6 D$ & D8 & $8 \mathrm{~A}$ & 84 & 72 & $2 \mathrm{~A}$ & 14 & $9 \mathrm{~F}$ & 88 & F9 & DC & 89 & $9 \mathrm{~A}$ \\
\hline & FB & 7C & $2 \mathrm{E}$ & C3 & $8 \mathrm{~F}$ & B8 & 65 & 48 & 26 & $\mathrm{CP}$ & 12 & $4 A$ & CE & E7 & D2 & 52 \\
\hline & $\mathrm{OC}$ & EO & $1 \mathrm{~F}$ & $\mathrm{EF}$ & 11 & 75 & 78 & 71 & A5 & $8 \mathrm{E}$ & 76 & 3D & BD & $\mathrm{BC}$ & 86 & 57 \\
\hline $\mathrm{C}$ & $\mathrm{OB}$ & 28 & $2 \mathrm{~F}$ & A3 & DA & D4 & E4 & OF & A9 & 27 & 53 & 04 & 1B & FC & $A C$ & H \\
\hline D & $7 \mathrm{~A}$ & 07 & $\mathrm{AE}$ & 63 & C5 & DB & E2 & EA & 94 & $8 \mathrm{~B}$ & C4 & D5 & 9D & F8 & 90 & $6 \mathrm{~B}$ \\
\hline $\mathrm{E}$ & B1 & OD & D6 & EB & C6 & $\mathrm{OE}$ & CF & $\mathrm{AD}$ & 08 & $4 E$ & D7 & E3 & 5D & 50 & $1 \mathrm{E}$ & B3 \\
\hline & $5 B$ & 23 & 38 & 34 & 68 & 46 & 03 & $8 \mathrm{C}$ & $\mathrm{DD}$ & $9 \mathrm{C}$ & $7 D$ & A0 & $C D$ & $1 \mathrm{~A}$ & 41 & \\
\hline
\end{tabular}

polynomials exist for every given degree $\mathrm{m}$ in $\mathrm{GF}\left(2^{m}\right)$ and only one of them is chosen as a standard for modulo reduction. For instance, $\mathrm{P}(\mathrm{x})=x^{4}+x+1$ is the standard irreducible polynomial used to reduce the product of the two polynomials $\mathrm{A}(\mathrm{x})=x^{3}$ $+x^{2}+1$ and $\mathrm{B}(\mathrm{x})=x^{2}+x$ in the field $\mathrm{GF}\left(2^{4}\right)$. Similarly for AES, the irreducible polynomial $\mathrm{P}(\mathrm{x})=x^{8}+x^{4}+x^{3}+x+1$ is used. It is part of the AES specification and plays an important role throughout the algorithm. Table 3.1 shows one primitive polynomial for every value of $\mathrm{m}$ in the range from $\mathrm{m}=2,3, \ldots, 128$. For example, the notation $(0,1,4)$ in the table refers to the polynomial $1+x+x^{4}$ in $\operatorname{GF}\left(2^{4}\right)$.

\subsubsection{Inversion in Galois Fields}

Inversion in $\mathrm{GF}\left(2^{8}\right)$ is one of the main operations of the Byte Substitution Layer, which contains the S-Boxes of AES. Finite field elements exhibit the property of having an inverse within the field [22]. For a given Galois field $\operatorname{GF}\left(2^{m}\right)$ and the corresponding irreducible reduction polynomial $\mathrm{P}(\mathrm{x})$, the inverse $A^{-1}$ of a non-zero element $\mathrm{A}$ in $\mathrm{GF}\left(2^{m}\right)$ is defined as:

$$
A^{-1}(x) \cdot A(x)=1 \bmod P(x)
$$

The inverse is computed using the Extended Euclidean Algorithm. From the 
Euclidean Algorithm, it is known that the Greatest common divisor (GCD) of any element in the AES field and the irreducible polynomial $\mathrm{P}(\mathrm{x})$ is always 1 . The EEA goal is to prove $\operatorname{gcd}\left(a_{0}, a_{1}\right)=\mathrm{s} . a_{0}+\mathrm{t} . a_{1}$, given the two integers $a_{0}$ and $a_{1}$. From the proof of EEA it is derived that $a^{-1}=\mathrm{t}$ [29]. Likewise in GF, $a_{0}$ is an element in $\mathrm{GF}\left(2^{8}\right), a_{1}$ is the irreducible polynomial and $a^{-1}$ is the inverse of the element $a_{0}$. This is how the multiplicative inverses are computed. A table which contains inverses of all the elements in $\operatorname{GF}\left(2^{8}\right)$ is given in Table 3.2. The elements are represented in hexadecimal notations and are mapped to their equivalent inverses. The entry for the field element 0 is mapped to itself since it has no inverse. Example, from the given table the inverse of element $\mathrm{B} 0$ is $0 \mathrm{C}$. The product of these two elements on modulo $\mathrm{P}(\mathrm{x})$ must be equal to 1.

$$
\begin{gathered}
B 0.0 C=1 \bmod P(x) \\
\text { where } B 0=x^{7}+x^{3}+x^{4} \\
0 C=x^{3}+x^{2} \\
P(x)=x^{8}+x^{4}+x^{3}+x+1
\end{gathered}
$$

\subsection{Structure of AES}

AES splits the 128-bits of plain text into 16 bytes of input. Every byte, i.e., $A_{0}$ to $A_{15}$ consist of 8-bits of data. The 8-bit binary number of every byte is passed to the S-boxes of the byte substitution layer. Fig. 3-2 illustrates the process flow of AES.

\subsubsection{Byte Substitution Layer}

The Byte Substitution layer is a row of sixteen parallel S-Boxes, each with a total of eight input and eight output bits. All the sixteen S-Boxes are identical, and each 


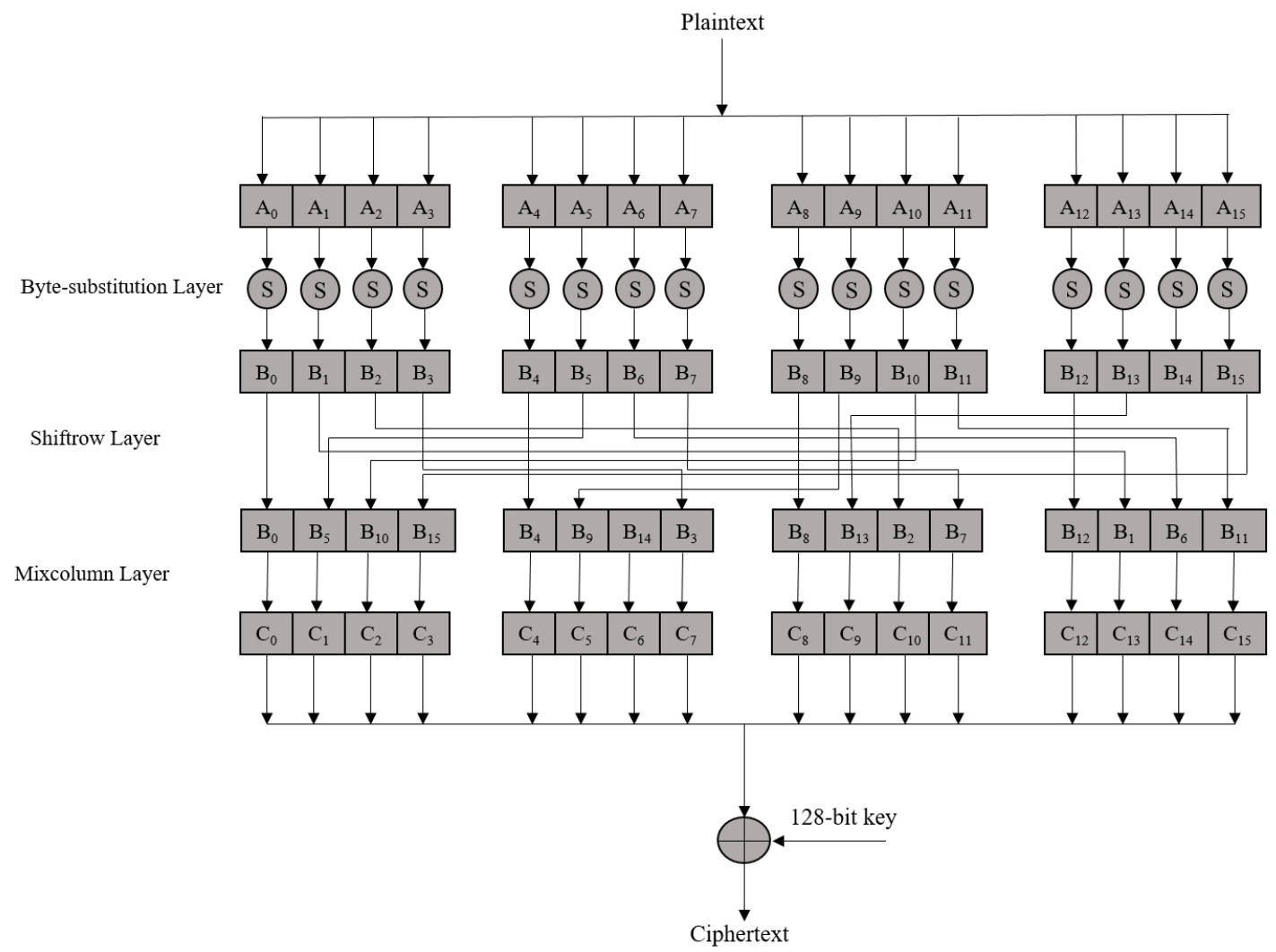

Figure 3-2: AES process flowchart

data byte $A_{i}$ is substituted by another byte $B_{i}$ at the outcome of S-box. Therefore the S-box operation can be denoted as $\mathrm{S}\left(A_{i}\right)=B_{i}$. The S-Box substitution is a one-to-one mapping of 256 input elements to one output [9]. They possess a strong algebraic structure.

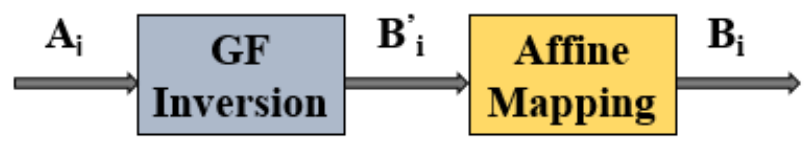

Figure 3-3: S-box construction

The construction involves a two-step mathematical transformation as shown in Fig. 3-4. In the first part, for each input element $A_{i}$, the inverse is computed as $A_{i}^{-1}=B_{i}^{1}$. The inverses can be directly substituted using the Multiplicative inverse 


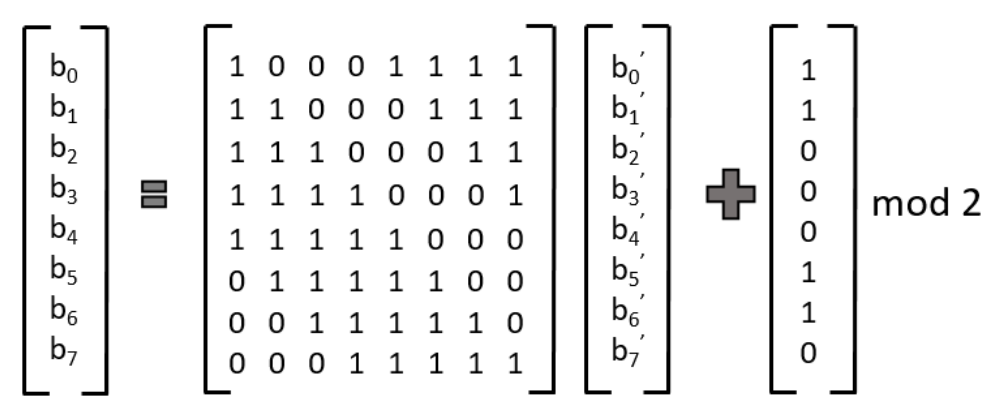

Figure 3-4: Affine Mapping

Table 3.3: S-box

\begin{tabular}{|c|c|c|c|c|c|c|c|c|c|c|c|c|c|c|c|c|}
\hline & 0 & 1 & 2 & 3 & 4 & 5 & 6 & 7 & 8 & 9 & 10 & 11 & 12 & 13 & 14 & 15 \\
\hline 0 & 63 & $7 C$ & 77 & 7B & F2 & $6 \mathrm{~B}$ & $6 \mathrm{~F}$ & C5 & 30 & 01 & 67 & $2 \mathrm{~B}$ & $\mathrm{FE}$ & D7 & $A B$ & 76 \\
\hline 1 & CA & 82 & C9 & 7D & FA & 59 & 47 & F0 & $A D$ & D4 & A2 & AF & $9 \mathrm{C}$ & A4 & 72 & Co \\
\hline 2 & B7 & FD & 93 & 26 & 36 & $3 F$ & F7 & CC & 34 & A5 & E5 & F1 & 71 & D8 & 31 & 15 \\
\hline 3 & 04 & C7 & 23 & C3 & 18 & 96 & 05 & $9 \mathrm{~A}$ & 07 & 12 & 80 & E2 & EB & 27 & B2 & 75 \\
\hline 4 & 09 & 83 & $2 C$ & 1A & 1B & $6 \mathrm{E}$ & $5 \mathrm{~A}$ & A0 & 52 & $3 B$ & D6 & B3 & 29 & E3 & $2 \mathrm{~F}$ & 84 \\
\hline 5 & 53 & D1 & 00 & ED & 20 & FC & B1 & 5B & $6 \mathrm{~A}$ & CB & $\mathrm{BE}$ & 39 & $4 \mathrm{~A}$ & $4 C$ & 58 & $\mathrm{CF}$ \\
\hline 6 & Do & EF & AA & FB & 43 & $4 \mathrm{D}$ & 33 & 85 & 45 & F9 & 02 & $7 F$ & 50 & $3 C$ & $9 \mathrm{~F}$ & A8 \\
\hline 7 & 51 & A3 & 40 & $8 \mathrm{~F}$ & 92 & 9D & 38 & F5 & $B C$ & B6 & DA & 21 & 10 & FF & F3 & D2 \\
\hline 8 & $\mathrm{CD}$ & $O C$ & 13 & EC & $5 \mathrm{~F}$ & 97 & 44 & 17 & C4 & A7 & 7E & 3D & 64 & 5D & 19 & 73 \\
\hline 9 & 60 & 81 & $4 F$ & DC & 22 & $2 \mathrm{~A}$ & 90 & 88 & 46 & $\mathrm{EE}$ & B8 & 14 & $\mathrm{DE}$ & $5 \mathrm{E}$ & OB & DB \\
\hline 10 & EO & 32 & $3 A$ & $O A$ & 49 & 06 & 24 & $5 C$ & $\mathrm{C} 2$ & D3 & $A C$ & 62 & 91 & 95 & E4 & 79 \\
\hline 11 & E7 & C8 & 37 & $6 \mathrm{D}$ & $8 \mathrm{D}$ & D5 & $4 \mathrm{E}$ & A9 & $6 \mathrm{C}$ & 56 & $\mathrm{~F} 4$ & EA & 65 & 7A & $\mathrm{AE}$ & 08 \\
\hline 12 & BA & 78 & 25 & $2 \mathrm{E}$ & $1 C$ & A6 & B4 & C6 & E8 & DD & 74 & $1 \mathrm{~F}$ & 4B & $\mathrm{BD}$ & 8B & $8 \mathrm{~A}$ \\
\hline 13 & 70 & $3 \mathrm{E}$ & B5 & 66 & 48 & 03 & F6 & $\mathrm{OE}$ & 61 & 35 & 57 & B9 & 86 & C1 & 1D & $9 \mathrm{E}$ \\
\hline 14 & E1 & F8 & 98 & 11 & 69 & D9 & $8 \mathrm{E}$ & 94 & $9 \mathrm{~B}$ & $1 \mathrm{E}$ & 87 & E9 & CE & 55 & 28 & DF \\
\hline 15 & $8 C$ & A1 & 89 & $O D$ & $\mathrm{R}$ & E6 & 42 & 68 & 41 & 99 & $2 \mathrm{D}$ & OF & BO & 54 & BB & 16 \\
\hline
\end{tabular}

table. Hence this transformation is known as Galois Field Inversion. In the second part of the layer, each of the output bytes of the Galois Field inversion, i.e., $B_{i}$ are multiplied by a constant bit matrix, followed by the addition of a constant 8-bit vector as shown in Fig. 3-4. This is known as Affine Mapping [12].

From the Fig. $3-4, B_{i}^{\prime}=\left(b_{7}^{1}, \ldots, b_{0}^{1}\right)$ is the bitwise vector representation of the final output of S-box. For example, let us assume $A_{i}=11001100$ as the input bit to the S-box. It is first converted into the hexadecimal notation which is CC. Then the equivalent inverse is fetched from the inverse table, that is $1 \mathrm{~B} .1 \mathrm{~B}$ is then converted into a binary representation (00011011) and is passed to $B_{i}^{\prime}$ (from left to right), and the matrix operation is done. The final output $B_{i}=01001011$ (4B) is substituted as output for $\mathrm{CC}$ in the S-box. By computing both steps for all 256 input elements of the S-Box, the Table. 3.3 can be constructed. 


\subsubsection{ShiftRows Layer}

In the shift rows layer, the sixteen output bits $\left(B_{0} \ldots B_{15}\right)$ from byte substitution layer are organized as a 4x4 matrix. Every 4 bytes are taken as a column vector in the input matrix. During the transformation, bytes from the second row are shifted to the right by three bytes in a cyclic manner. Similarly, the third row is shifted by two bytes and fourth row by one byte. The first row of the output matrix remains unchanged as shown in Fig. 3-5.

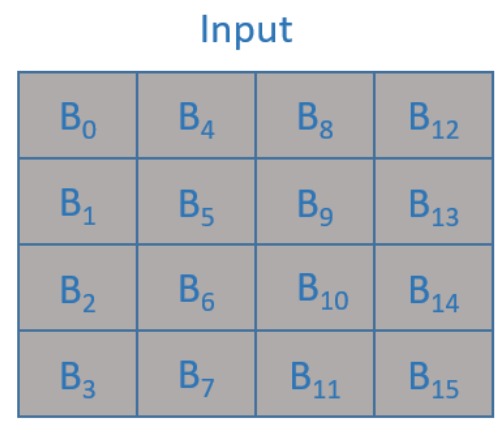

\begin{tabular}{|c|c|c|c|}
\hline $\mathrm{B}_{0}$ & $\mathrm{~B}_{4}$ & $\mathrm{~B}_{8}$ & $\mathrm{~B}_{12}$ \\
\hline $\mathrm{B}_{5}$ & $\mathrm{~B}_{9}$ & $\mathrm{~B}_{13}$ & $\mathrm{~B}_{1}$ \\
\hline $\mathrm{B}_{10}$ & $\mathrm{~B}_{14}$ & $\mathrm{~B}_{2}$ & $\mathrm{~B}_{6}$ \\
\hline $\mathrm{B}_{15}$ & $\mathrm{~B}_{3}$ & $\mathrm{~B}_{7}$ & $\mathrm{~B}_{11}$ \\
\hline
\end{tabular}

Figure 3-5: Input and Output matrices

\subsubsection{MixColumn Layer}

As the name suggests, the Mixcolumn layer combines each column of the state matrix. Four bytes from every column of the output state matrix (Shiftrows layer) are considered as input to the mixcolumn layer. This column vector is multiplied by a fixed $4 \times 4$ matrix which consists of constant values as shown in the Fig. 3-6. The output bytes $C_{0} \ldots C_{15}$ are a result of this matrix multiplication. All the arithmetic operations are performed in the Galois field. The fixed constants in the matrix are represented by their hexadecimal notations. For example, the element "03" is the hexadecimal representation of the bit (00000011) whose value equals to $x+1$ in the Galois fields. Mixcolumn layer is not present in the last round of AES. 


$\left[\begin{array}{l}\mathrm{C}_{0} \\ \mathrm{C}_{1} \\ \mathrm{C}_{2} \\ \mathrm{C}_{3}\end{array}\right]$ 吕 $\left[\begin{array}{llll}02 & 03 & 01 & 01 \\ 01 & 02 & 03 & 01 \\ 01 & 01 & 02 & 03 \\ 03 & 01 & 01 & 02\end{array}\right]\left[\begin{array}{l}\mathrm{B}_{0} \\ \mathrm{~B}_{5} \\ \mathrm{~B}_{10} \\ \mathrm{~B}_{15}\end{array}\right]$

Figure 3-6: Mixcolumn Layer

\subsubsection{Key Addition Layer}

In an AES of 128-bit key size, initially, the key is generated by an RNG (Random number generator) and processed through 10 rounds. AES has key addition layer in every round (sub key is in every round of AES). In the first round, the generated 128-bits are split into four 32-bits and every 32-bit is stored in array denoted as W as shown in Fig. 3-7. From the second round till the final round, the other array elements are computed as: $\mathrm{W}[4 \mathrm{i}]=\mathrm{W}[4(\mathrm{i} 1)]+\mathrm{g}(\mathrm{W}[4 \mathrm{i} 1])$; where $\mathrm{i}=1, \ldots$. . , 10,(no. of rounds)

$\mathrm{g}$ is a nonlinear function with a 32-bits input and output. The remaining three arrays of the sub-key are computed recursively as: $\mathrm{W}[4 \mathrm{i}+\mathrm{j}]=\mathrm{W}[4 \mathrm{i}+\mathrm{j} 1]+\mathrm{W}[4(\mathrm{i} 1)+$ $\mathrm{j}]$, where $\mathrm{i}=1, \ldots$. . 10 and $\mathrm{j}=1,2,3$. The function rotates its input bits and performs a byte-wise S-Box substitution, and adds (XOR's) a round coefficient RC to it. The round coefficient is an element of the Galois field $G F\left(2^{8}\right)$, i.e, an 8-bit value. It is only added to the leftmost byte in the function $\mathrm{g}()$. The round coefficients for every round are standard values specified below $\mathrm{RC}[1]=x_{0}=(00000001), \mathrm{RC}[2]=$ $x_{1}=(00000010), \mathrm{RC}[3]=x_{2}=(00000100), \ldots \mathrm{RC}[10]=x_{9}=(00110110)$. where $\mathrm{i}$ $=1, \ldots, 10,($ no. of rounds) $[24]$ 


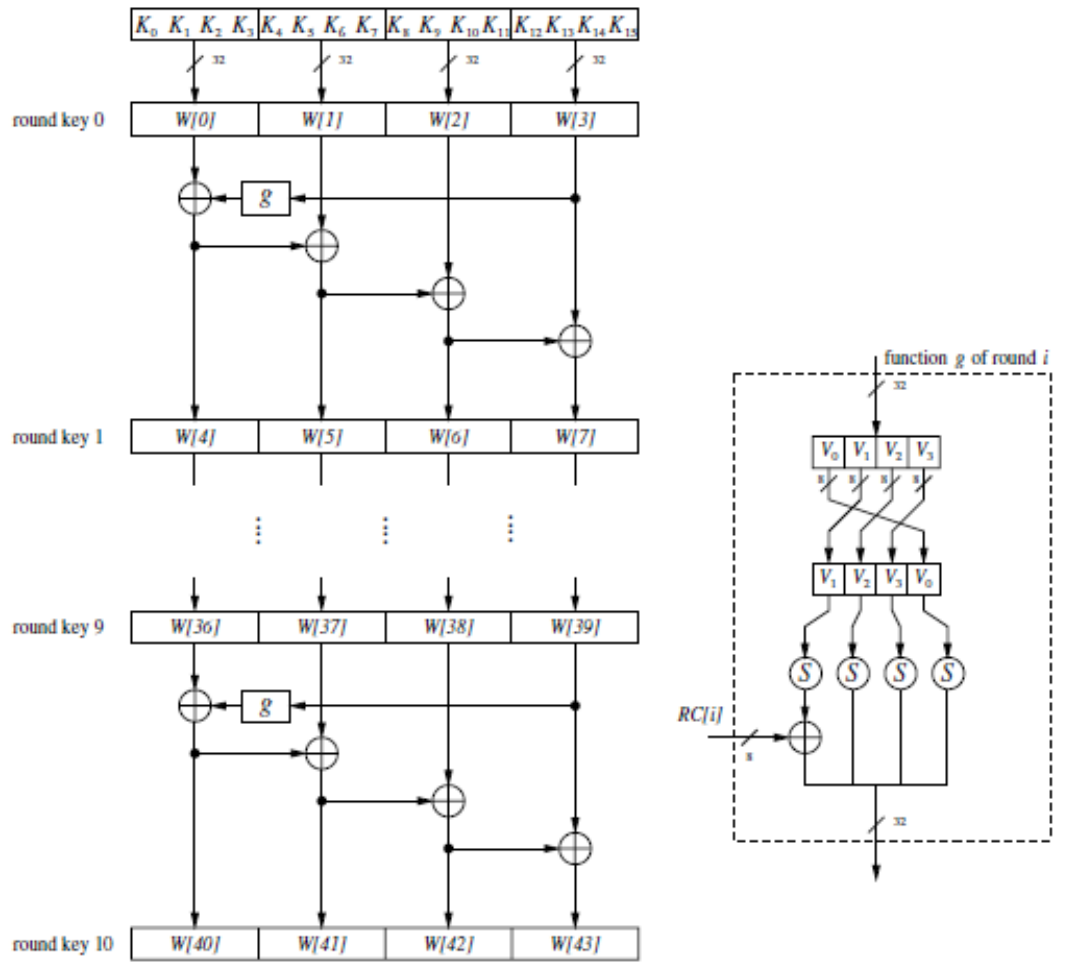

Figure 3-7: AES key scedule for 128-bit key size [24]

\subsection{AES Decryption}

Decryption of AES is the encryption in reverse order. The operations performed in each of the AES decryption layers are the inverse of the ones in encryption as shown in Fig. 3-8. It starts with the cipher text added to the last sub-key of the key addition layer. The output of this is then processed through the Inverse mixcolumn layer, inverse shiftrow layer and finally to the inverse byte substitution layer. The first round of decryption doesn't contain the mixcolumn layer since it is not present in last round of AES encryption. Thus, the AES decryption starts with the output of Key addition layer sent to the Inverse Shift rows Layer. 


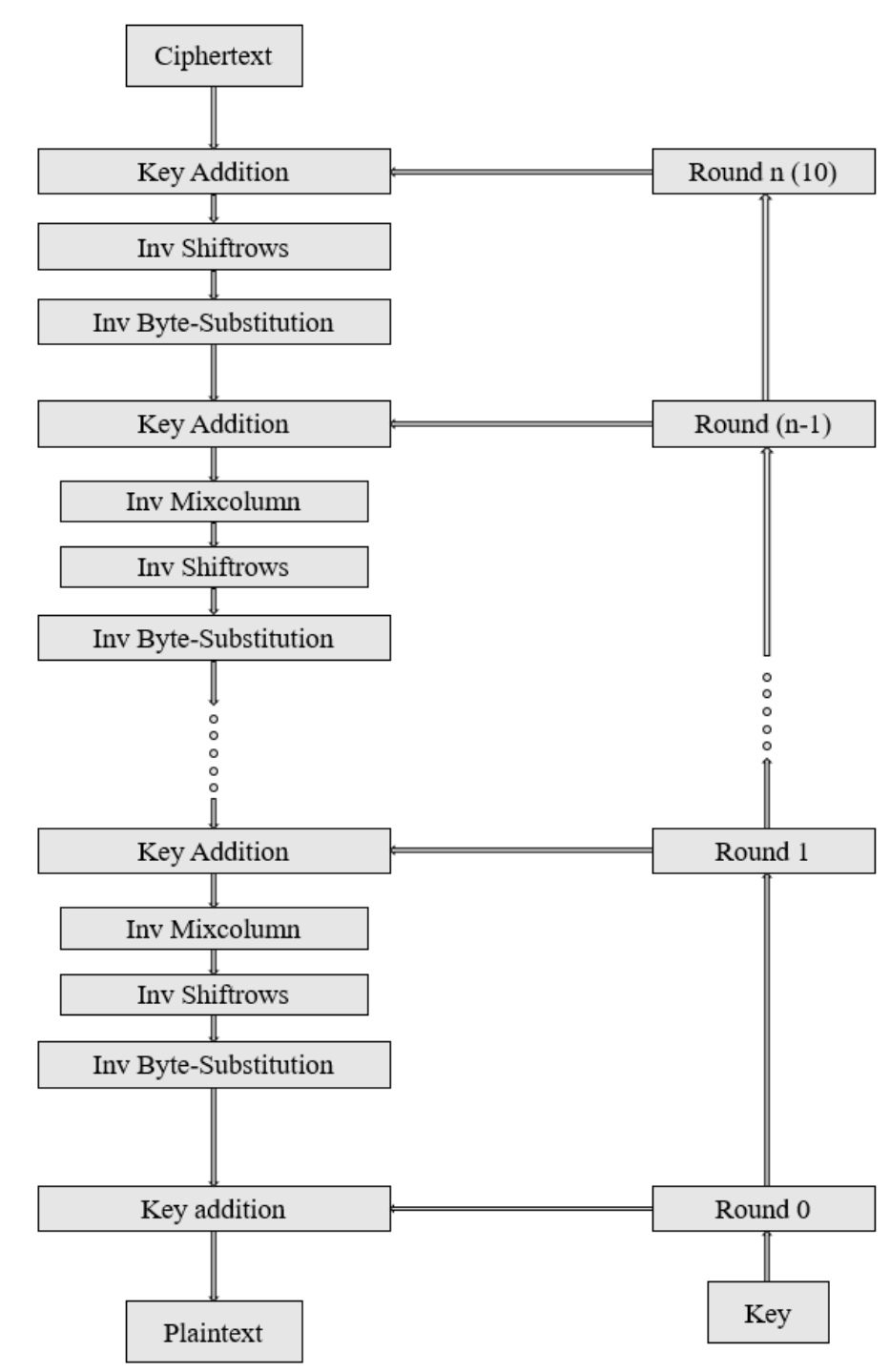

Figure 3-8: AES decryption block diagram

\subsubsection{Inverse MixColumn Sublayer}

The inverse MixColumn step is applied to the input bytes after the addition of the subkey ( first decryption round being an exception). The operation is reversed by multiplying the input bytes by an inverse matrix with constant entries. Example of the first four input bytes $\left(C_{0}, C_{1}, C_{2}, C_{3}\right)$ multiplied by the constant matrix is shown in Fig. 3-9. Multiplication and addition of the coefficients are done in $G F\left(2^{8}\right)$, same as the mixcolumn layer in encryption. 


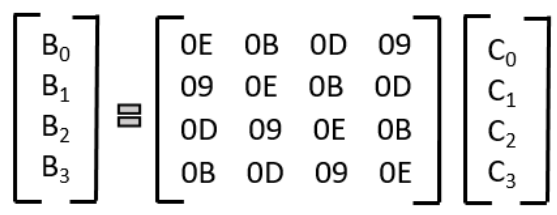

Figure 3-9: Inverse Mixcolumn Layer

\subsubsection{Inverse ShiftRows Sublayer}

This operation is performed by shifting the rows of the input matrix in the opposite direction. The output bytes $\left(B_{0} \ldots B_{15}\right)$ of the inverse mix column layer are arranged in the $4 \times 4$ matrix. The first row of the matrix remains unchanged; the second row is shifted to the right by three bytes in a cyclic manner. Similarly, the third row is shifted by two bytes and fourth row by one byte to the right. The outcome of the inverse shift rows layer is shown in Fig. 3-10

Input
\begin{tabular}{|c|c|c|c|}
\hline $\mathrm{B}_{0}$ & $\mathrm{~B}_{4}$ & $\mathrm{~B}_{8}$ & $\mathrm{~B}_{12}$ \\
\hline $\mathrm{B}_{1}$ & $\mathrm{~B}_{5}$ & $\mathrm{~B}_{9}$ & $\mathrm{~B}_{13}$ \\
\hline $\mathrm{B}_{2}$ & $\mathrm{~B}_{6}$ & $\mathrm{~B}_{10}$ & $\mathrm{~B}_{14}$ \\
\hline $\mathrm{B}_{3}$ & $\mathrm{~B}_{7}$ & $\mathrm{~B}_{11}$ & $\mathrm{~B}_{15}$ \\
\hline
\end{tabular}

Output
\begin{tabular}{|c|c|c|l|}
\hline $\mathrm{B}_{0}$ & $\mathrm{~B}_{4}$ & $\mathrm{~B}_{8}$ & $\mathrm{~B}_{12}$ \\
\hline $\mathrm{B}_{13}$ & $\mathrm{~B}_{1}$ & $\mathrm{~B}_{5}$ & $\mathrm{~B}_{9}$ \\
\hline $\mathrm{B}_{10}$ & $\mathrm{~B}_{14}$ & $\mathrm{~B}_{2}$ & $\mathrm{~B}_{6}$ \\
\hline $\mathrm{B}_{7}$ & $\mathrm{~B}_{11}$ & $\mathrm{~B}_{15}$ & $\mathrm{~B}_{3}$ \\
\hline
\end{tabular}

Figure 3-10: Matrices in Inverse Shiftrows

\subsubsection{Inverse Byte Substitution Layer}

In this layer, all the operations are reversed to get inverse S-boxes. The output bytes from the Inverse shiftrows layer go through two transformations, the first step is the affine mapping and the second one is the Galois inversion. Similar to encryption, the input bits are multiplied by an inverse matrix in affine mapping followed by the addition of a constant 8-bit vector shown in Fig. 3-11 


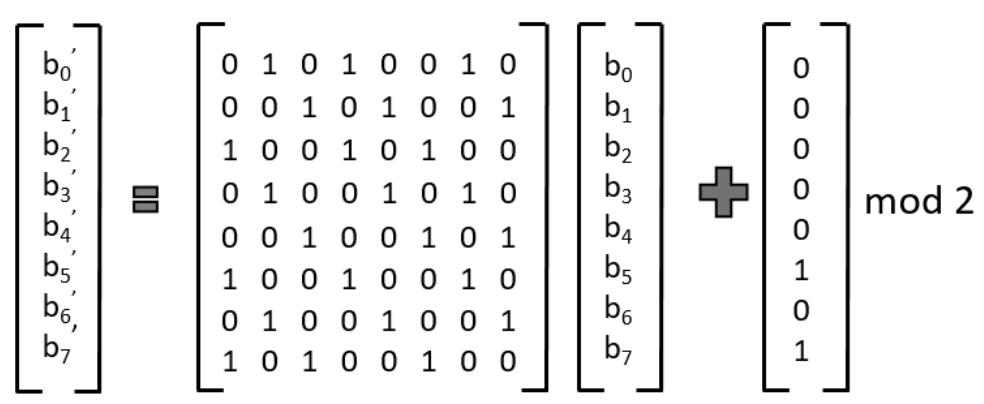

Figure 3-11: Inverse Affine Mapping

Table 3.4: Inverse S-box

\begin{tabular}{|c|c|c|c|c|c|c|c|c|c|c|c|c|c|c|c|c|}
\hline & 0 & 1 & 2 & 3 & 4 & 5 & 6 & 7 & 8 & 9 & 10 & 11 & 12 & 13 & 14 & 15 \\
\hline 0 & 52 & 09 & $6 \mathrm{~A}$ & D5 & 30 & 36 & A5 & 38 & BF & 40 & A3 & $9 \mathrm{E}$ & 81 & F3 & D7 & FB \\
\hline 1 & 7C & E3 & 39 & 82 & $9 \mathrm{~B}$ & $2 F$ & FF & 87 & 34 & $8 \mathrm{E}$ & 43 & 44 & $\mathrm{C} 4$ & $\mathrm{DE}$ & E9 & $\mathrm{CB}$ \\
\hline 2 & 54 & 7B & 94 & 32 & A6 & $\mathrm{C} 2$ & 23 & $3 D$ & $\mathrm{EE}$ & $4 C$ & 95 & $\mathrm{OB}$ & 42 & FA & C3 & $4 \mathrm{E}$ \\
\hline 3 & 08 & $2 \mathrm{E}$ & A1 & 66 & 28 & D9 & 24 & B2 & 76 & $5 B$ & A2 & 49 & $6 \mathrm{D}$ & $8 \mathrm{~B}$ & D1 & 25 \\
\hline 4 & 72 & F8 & F6 & 64 & 86 & 68 & 98 & 16 & D4 & A4 & $5 \mathrm{C}$ & $\mathrm{CC}$ & 5D & 65 & B6 & 92 \\
\hline 5 & $6 C$ & 70 & 48 & 50 & $\mathrm{FD}$ & ED & B9 & DA & $5 \mathrm{E}$ & 15 & 46 & 57 & A7 & $8 \mathrm{D}$ & 9D & 84 \\
\hline 6 & 90 & D8 & $A B$ & 00 & $8 \mathrm{C}$ & $B C$ & D3 & $0 \mathrm{~A}$ & F7 & E4 & 58 & 05 & B8 & B3 & 45 & 06 \\
\hline 7 & DO & $2 C$ & $1 \mathrm{E}$ & $8 \mathrm{~F}$ & CA & $3 F$ & OF & 02 & C1 & AF & $\mathrm{BD}$ & 03 & 01 & 13 & $8 \mathrm{~A}$ & $6 \mathrm{~B}$ \\
\hline 8 & $3 A$ & 91 & 11 & 41 & $4 \mathrm{~F}$ & 67 & DC & EA & 97 & F2 & CF & CE & F0 & B4 & E6 & 73 \\
\hline 9 & 96 & $A C$ & 74 & 22 & E7 & $A D$ & 35 & 85 & E2 & F9 & 37 & E8 & $1 \mathrm{C}$ & 75 & DF & $6 \mathrm{E}$ \\
\hline 10 & 47 & F1 & $1 \mathrm{~A}$ & 71 & 1D & 29 & C5 & 89 & $6 \mathrm{~F}$ & B7 & 62 & $\mathrm{OE}$ & AA & 18 & $\mathrm{BE}$ & 1B \\
\hline 11 & FC & 56 & $3 E$ & 4B & C6 & D2 & 79 & 20 & $9 \mathrm{~A}$ & DB & $\mathrm{CO}$ & $\mathrm{FE}$ & 78 & $\mathrm{CD}$ & $5 A$ & F4 \\
\hline 12 & $1 \mathrm{~F}$ & DD & A8 & 33 & 88 & 07 & C7 & 31 & B1 & 12 & 10 & 59 & 27 & 80 & EC & $5 F$ \\
\hline 13 & 60 & 51 & 7F & A9 & 19 & B5 & $4 A$ & OD & $2 \mathrm{D}$ & E5 & 7A & $9 \mathrm{~F}$ & 93 & C9 & $9 \mathrm{C}$ & EF \\
\hline 14 & A0 & EO & 3B & $4 D$ & $\mathrm{AE}$ & $2 A$ & F5 & BO & $\mathrm{C} 8$ & EB & BB & $3 C$ & 83 & 53 & 99 & 61 \\
\hline 15 & 17 & $2 \mathrm{~B}$ & 04 & $7 E$ & BA & 77 & D6 & 26 & E1 & 69 & 14 & 63 & 55 & 21 & $O C$ & $7 D$ \\
\hline
\end{tabular}

Where $B_{i}$ is the input and $B_{i}^{1}$ is the output of affine mapping. In the second step, bits in vector $B_{i}^{1}$ undergo Galois inversion. All the multiplications and inversions are computed with the fixed reduction polynomial $\mathrm{P}(\mathrm{x})=x^{8}+x^{4}+x^{3}+x+1$.

The inverse of the corresponding bit is substituted from the multiplicative inverse table as $A_{i}=\left(B_{i}^{1}\right)^{-1}$. All the 256 elements are thus computed using the expression $A_{i}=S^{-1}\left(B_{i}\right)$ and form an inverse S-box table with their hexadecimal notations as shown in Table 3.4. Therefore by the end of inverse byte substitution layer, the ciphertext is decrypted to plaintext. 


\section{Chapter 4}

\section{Customized Encryption Approach}

\subsection{Introduction}

As described in Chapter 3, the standard polynomial $x^{8}+x^{4}+x^{3}+x+1$ used in AES, is one among the many irreducible polynomials [30] that exist for $G F\left(2^{8}\right)$. For every $\mathrm{GF}\left(2^{m}\right)$, the polynomials exist between $2^{m}$ and $2^{(m+1)}$. Likewise, sixteen such irreducible polynomials exist between $2^{8}$ and $2^{9}$ for $G F\left(2^{8}\right)$ [30]. The list of polynomial equations are presented in the Table 4.1

The 256 elements form multiplicative inverse tables with all the polynomials, using the same Extended Euclidean Algorithm (EEA). The process is described in two steps:

- In the first step, GCD of the element and polynomial equation is computed.

- Using the EEA, the inverse of every element is calculated, and sixteen multiplicative inverse tables are created.

This process results in the computation of sixteen S-boxes and inverse S-boxes. Using Python, we computed the boxes presented from Table 4.2 to Table 4.17

As compared to the AES framework, the customized protocol differs in the following ways:

- The Byte-substitution layer retrieves one of the sixteen S-boxes based on the 
Table 4.1: Irreducible Polynomial Equations

\begin{tabular}{|l|l|}
\hline & Polynomial Equations \\
\hline 1 & $x^{8}+x^{4}+x^{3}+x^{2}+1$ \\
\hline 2 & $x^{8}+x^{5}+x^{3}+x+1$ \\
\hline 3 & $x^{8}+x^{5}+x^{3}+x^{2}+1$ \\
\hline 4 & $x^{8}+x^{6}+x^{3}+x^{2}+1$ \\
\hline 5 & $x^{8}+x^{6}+x^{4}+x^{3}+x^{2}+x+1$ \\
\hline 6 & $x^{8}+x^{6}+x^{5}+x+1$ \\
\hline 7 & $x^{8}+x^{6}+x^{5}+x^{2}+1$ \\
\hline 8 & $x^{8}+x^{6}+x^{5}+x^{3}+1$ \\
\hline 9 & $x^{8}+x^{6}+x^{5}+x^{4}+1$ \\
\hline 10 & $x^{8}+x^{7}+x^{2}+x+1$ \\
\hline 11 & $x^{8}+x^{7}+x^{3}+x^{2}+1$ \\
\hline 12 & $x^{8}+x^{7}+x^{5}+x^{3}+1$ \\
\hline 13 & $x^{8}+x^{7}+x^{6}+x+1$ \\
\hline 14 & $x^{8}+x^{7}+x^{6}+x^{3}+x^{2}+x+1$ \\
\hline 15 & $x^{8}+x^{7}+x^{6}+x^{5}+x^{2}+x+1$ \\
\hline 16 & $x^{8}+x^{7}+x^{6}+x^{5}+x^{4}+x^{2}+1$ \\
\hline
\end{tabular}

Table 4.2: S-box and Inverse S-box for $x^{8}+x^{4}+x^{3}+x^{2}+1$ 
Table 4.3: S-box and Inverse S-box for $x^{8}+x^{5}+x^{3}+x+1$

\begin{tabular}{|c|c|c|c|c|c|c|c|c|c|c|c|c|c|c|c|c|}
\hline & 0 & 1 & 2 & 3 & 4 & 5 & 6 & 7 & 8 & 9 & A & B & C & D & $E$ & $\mathrm{~F}$ \\
\hline 0 & 63 & $7 C$ & $7 \mathrm{E}$ & $8 \mathrm{~A}$ & $7 F$ & 27 & 97 & 73 & $\mathrm{FF}$ & $8 \mathrm{~F}$ & D3 & 36 & $8 B$ & 91 & $6 \mathrm{~B}$ & $\mathrm{~A} 0$ \\
\hline 1 & $2 D$ & DD & 87 & $\mathrm{C} 1$ & $3 B$ & B2 & $5 B$ & $2 E$ & 17 & 55 & $1 \mathrm{~A}$ & DB & 67 & 50 & 10 & E5 \\
\hline 2 & D6 & 02 & $\mathrm{AE}$ & 30 & 83 & D7 & 32 & $8 D$ & $4 \mathrm{~F}$ & 16 & 19 & 71 & ED & F4 & 57 & EA \\
\hline 3 & 59 & 06 & 78 & 09 & 4D & E1 & $3 \mathrm{~F}$ & D4 & F3 & 58 & 68 & 93 & 48 & 25 & 20 & $2 C$ \\
\hline 4 & $2 B$ & 45 & 41 & D8 & 85 & $5 E$ & CA & BD & 13 & 49 & AB & 69 & CB & 33 & 86 & $1 \mathrm{C}$ \\
\hline 5 & 75 & 08 & D9 & BF & $\mathrm{CC}$ & BA & $6 \mathrm{~A}$ & $4 \mathrm{~A}$ & 24 & F1 & A8 & 77 & 79 & 40 & 35 & E2 \\
\hline 6 & EC & 96 & D1 & $5 F$ & $\mathrm{EE}$ & $A D$ & C4 & 54 & 74 & C6 & BO & 3D & DF & A7 & $2 \mathrm{~A}$ & FO \\
\hline 7 & B9 & 07 & $6 \mathrm{C}$ & 21 & E6 & A2 & $1 \mathrm{~B}$ & F2 & 64 & F6 & D2 & 53 & C2 & 92 & 56 & $5 \mathrm{C}$ \\
\hline 8 & 47 & 89 & 70 & $4 C$ & EO & 84 & BE & $2 \mathrm{~F}$ & 82 & 15 & FD & EF & B7 & $8 \mathrm{C}$ & $O C$ & 43 \\
\hline 9 & $\mathrm{CP}$ & $9 \mathrm{~F}$ & E4 & A3 & 95 & 5D & 66 & CE & 37 & $\mathrm{OF}$ & 4B & 05 & 03 & $1 \mathrm{E}$ & DC & $\mathrm{CO}$ \\
\hline A & FA & 28 & 44 & CF & $3 E$ & 88 & OD & $\mathrm{FE}$ & 26 & $6 \mathrm{D}$ & 1D & 80 & E7 & $8 \mathrm{E}$ & 65 & C5 \\
\hline B & 52 & 12 & B8 & C3 & 14 & $O A$ & FB & $3 C$ & $6 \mathrm{E}$ & 46 & 60 & 00 & DA & B5 & 31 & DO \\
\hline C & A4 & $5 A$ & OB & $9 \mathrm{D}$ & $3 A$ & F5 & 7D & B4 & A5 & 29 & 04 & EB & 22 & 81 & F8 & 94 \\
\hline D & $7 A$ & AA & 23 & $\mathrm{BC}$ & 18 & B6 & $\mathrm{DE}$ & AC & AF & $9 \mathrm{E}$ & 01 & 99 & C7 & 9A & 38 & $1 F$ \\
\hline$E$ & $9 \mathrm{C}$ & E3 & 51 & 7B & 76 & 62 & 42 & 61 & A1 & B1 & 11 & $0 \mathrm{E}$ & $C D$ & $6 \mathrm{~F}$ & 39 & E8 \\
\hline & 72 & F7 & A9 & A6 & BB & 34 & E9 & $4 \mathrm{E}$ & B3 & 98 & $9 \mathrm{~B}$ & 90 & F9 & D5 & FC & $\mathrm{C} 8$ \\
\hline
\end{tabular}

\begin{tabular}{|c|c|c|c|c|c|c|c|c|c|c|c|c|c|c|c|c|}
\hline & 0 & 1 & 2 & 3 & 4 & 5 & 6 & 1 & 8 & 9 & A & B & C & D & $E$ & F \\
\hline 0 & BB & DA & 21 & $9 \mathrm{C}$ & CA & $9 \mathrm{~B}$ & 31 & 71 & 51 & 33 & B5 & $\mathrm{C} 2$ & $8 \mathrm{E}$ & A6 & EB & 99 \\
\hline 1 & $1 \mathrm{E}$ & EA & B1 & 48 & B4 & 89 & 29 & 18 & D4 & $2 A$ & $1 \mathrm{~A}$ & 76 & $4 \mathrm{~F}$ & AA & $9 D$ & DF \\
\hline 2 & $3 \mathrm{E}$ & 73 & $\mathrm{CC}$ & D2 & 58 & $3 D$ & A8 & 05 & A1 & $\mathrm{CP}$ & $6 \mathrm{E}$ & 40 & $3 F$ & 10 & 17 & 87 \\
\hline 3 & 23 & BE & 26 & 4D & F5 & $5 \mathrm{E}$ & $\mathrm{OB}$ & 98 & DE & $\mathrm{EE}$ & $\mathrm{C} 4$ & 14 & B7 & $6 \mathrm{~B}$ & A4 & 36 \\
\hline 4 & $5 D$ & 42 & E6 & $8 F$ & A2 & 41 & B9 & 80 & $3 C$ & 49 & 57 & $9 \mathrm{~A}$ & 83 & 34 & F7 & 28 \\
\hline 5 & 1D & E2 & B0 & 7B & 67 & 19 & 7E & $2 \mathrm{E}$ & 39 & 30 & $\mathrm{C} 1$ & 16 & $7 F$ & 95 & 45 & 63 \\
\hline 6 & BA & E7 & E5 & 00 & 78 & $\mathrm{AE}$ & 96 & $1 \mathrm{C}$ & $3 \mathrm{~A}$ & $4 B$ & 56 & $0 \mathrm{E}$ & 72 & A9 & B8 & ED \\
\hline 7 & 82 & $2 B$ & F0 & 07 & 68 & 50 & E4 & $5 B$ & 32 & $5 C$ & D0 & E3 & 01 & C6 & 02 & 04 \\
\hline 8 & $A B$ & $C D$ & 88 & 24 & 85 & 44 & $4 \mathrm{E}$ & 12 & A5 & 81 & 03 & $O C$ & $8 \mathrm{D}$ & 27 & $A D$ & 09 \\
\hline 9 & FB & $O D$ & 7D & 3B & $\mathrm{CF}$ & 94 & 61 & 06 & F9 & DB & DD & FA & EO & C3 & D9 & 91 \\
\hline A & OF & E8 & 75 & 93 & $\mathrm{CO}$ & C8 & F3 & $6 \mathrm{D}$ & $5 \mathrm{~A}$ & $\mathrm{~F} 2$ & D1 & $4 \mathrm{~A}$ & D7 & 65 & 22 & D8 \\
\hline B & $6 \mathrm{~A}$ & E9 & 15 & F8 & C7 & $B D$ & D5 & $8 \mathrm{C}$ & B2 & 70 & 55 & F4 & D3 & 47 & 86 & 53 \\
\hline C & $9 \mathrm{~F}$ & 13 & 7C & B3 & 66 & $\mathrm{AF}$ & 69 & DC & $\mathrm{FF}$ & 90 & 46 & $4 C$ & 54 & EC & 97 & A3 \\
\hline D & BF & 62 & 7A & $O A$ & 37 & FD & 20 & 25 & 43 & 52 & $B C$ & $1 \mathrm{~B}$ & $9 \mathrm{E}$ & 11 & D6 & $6 C$ \\
\hline$E$ & 84 & 35 & $5 F$ & E1 & 92 & $1 \mathrm{~F}$ & 74 & $A C$ & $E-$ & F6 & 2 & CB & 60 & $2 C$ & 64 & $8 B$ \\
\hline & & & & & & $\mathrm{cs}$ & & & & & & & & & & \\
\hline
\end{tabular}

Table 4.4: S-box and Inverse S-box for $x^{8}+x^{5}+x^{3}+x^{2}+1$

\begin{tabular}{|c|c|c|c|c|c|c|c|c|c|c|c|c|c|c|c|c|}
\hline & & & & & & & & & & & & & & & & \\
\hline & & & & & & & 38 & 48 & & 85 & A & & 96 & F5 & & \\
\hline & & & & & & & & & & & & & & & & \\
\hline & & & & & & & & & & & & & & & & \\
\hline & & & & & & & & & & & & & & & & \\
\hline & & & & & & & & & & & & & & & & \\
\hline & & & & & & & & & & & & & & & & \\
\hline & & & & & & & & & & & & & & & & \\
\hline & & & & & & & & & & & & & & & & \\
\hline & & & & & & & & & & & & & & & & \\
\hline & & & & & & & & & & & & & & & & \\
\hline & & & & & & & & & & & & & & & & \\
\hline & & & & & & & & & & 4D & & & & & & \\
\hline & & & & & & & & & D & & 3 & & & & & \\
\hline & & & & & & & & & & & & & & & & \\
\hline & & & 46 & & 4 & 40 & 79 & 4 & 0 & $F$ & 17 & 32 & 81 & 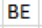 & & \\
\hline & & & & & & & & & & & & & & & & \\
\hline
\end{tabular}

\begin{tabular}{|c|c|c|c|c|c|c|c|c|c|c|c|c|c|c|c|c|}
\hline & & & & & & & & & & & & & & & & \\
\hline & & & 88 & 1 & & & & 2 & ) & BO & $9 \mathrm{E}$ & 55 & $5 \mathrm{E}$ & OF & & \\
\hline & & & & & & & & & & & & & & & & \\
\hline & & & & & & & & & 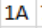 & & 18 & & & & & \\
\hline & & & & & & & & & & & & & & & & \\
\hline & & & & & & & & & 7 & 0 & & & & & & \\
\hline & & & & & & & & & & & & & & & & \\
\hline & & & & & & & & & & & & & & & & \\
\hline & & & & & & & & & & & & & & & & \\
\hline & & & & & & & & & & & & & & & & \\
\hline & & & & & & & & & 7 & $t$ & & 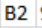 & & 3 & & \\
\hline & & & & & & & & & & & & & & & & \\
\hline & & & & & & & & & 6 & 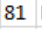 & & $8 F$ & 3 & & & \\
\hline & & & & & & & & & & & 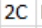 & & & D & & \\
\hline & & & & & & & & & & & & & & & & \\
\hline & & & & & & & & & $\vec{A}$ & f & 3 & 80 & 76 & 60 & 16 & \\
\hline & & & & & & & & & & & & & & & & \\
\hline
\end{tabular}

Table 4.5: S-box and Inverse S-box for $x^{8}+x^{6}+x^{3}+x^{2}+1$

\begin{tabular}{|c|c|c|c|c|c|c|c|c|c|c|c|c|c|c|c|c|}
\hline & & & & & & & & & & & & & & & & \\
\hline & & & & & & & & & 49 & 04 & 3 & $\mathrm{FC}$ & $6 \mathrm{E}$ & EF & & \\
\hline & & & & & & & & & t & & & ti & 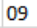 & & & \\
\hline & & & & & & & & & & & & & & & & \\
\hline & & & & & & & & & 7 & & & 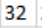 & 20 & 7 & & \\
\hline & & & & & & & & & & & & & & & & \\
\hline & & & & & & & & & 1 & & & & E2 & ED & F & \\
\hline & & & & & & & & & & & & & & & & \\
\hline & & & & & & & & & & & 3 & & & & & \\
\hline & & & & & & & & & & & & 3 & & & & \\
\hline & & & & & & & & & & & & & 69 & & & \\
\hline & & & & & & & & 4 & 8 & $7 E$ & 0 & 18 & c & $A A$ & 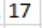 & \\
\hline & & & & & & & & & & & & & & & & \\
\hline & $\mathrm{Fe}$ & & & & & 2 & 9 & & & 3 & $c$ & 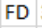 & 3 & & 51 & \\
\hline & & & & & & & & & & & & & & & & \\
\hline & 46 & & & & & & & & & & 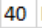 & & & & & \\
\hline & & & & & & & & & & & & & & & & \\
\hline
\end{tabular}

\begin{tabular}{|l|l|l|llllllllllllllllllll|} 
& 0 & 1 & 2 & 3 & 4 & 5 & 6 & 7 & 8 & 9 & A & B & C & D & E & $F$ \\
\hline
\end{tabular} \begin{tabular}{l|lllllllllllllllllll}
\hline 0 & 87 & E3 & B8 & 37 & 09 & AA & 91 & 67 & AC & $1 C$ & DC & 60 & $9 B$ & B1 & 97 & BC \\
\hline
\end{tabular}

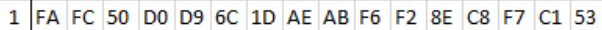

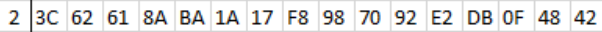
\begin{tabular}{l|llllllllllllllllllll}
3 & A5 & 58 & $3 B$ & C9 & $4 E$ & 81 & CC & EC & 65 & 13 & DE & F9 & 80 & 88 & 74 & EF
\end{tabular} 4 EA 6 D $C 4$\begin{tabular}{llllll|l|l|l|l|l|l|l}
59 & 18 & $9 D$ & E0 & 89 & A7 & 08 & B0 & DF & $1 F$ & 02 & 26 & BF
\end{tabular} \begin{tabular}{l|llll|l|l|l|l|l|l|l|l|l|l|l|l}
5 & 19 & CE & $C 5$ & 99 & $2 F$ & 35 & $2 D$ & 03 & D8 & 11 & C7 & 32 & A3 & A6 & C3 & FD
\end{tabular} $\begin{array}{llllllllllllllllll}6 & 2 \mathrm{E} & 39 & 72 & 00 & 90 & \mathrm{FB} & 75 & 41 & \mathrm{D} 5 & 9 \mathrm{C} & \mathrm{BE} & 86 & \mathrm{E} 8 & 4 \mathrm{D} & 0 \mathrm{C} & 3 \mathrm{~F}\end{array}$

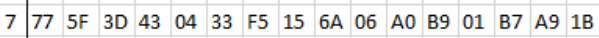
\begin{tabular}{lllllllllll|lll|l|l|l|l}
8 & D6 & 28 & 85 & 68 & $2 \mathrm{C}$ & A4 & F4 & 9E & 4 A & A8 & $5 B$ & 57 & E6 & 2B & 27 & B4
\end{tabular} \begin{tabular}{l|llllllllllllllllll}
9 & D2 & 79 & E1 & 47 & AF & 46 & 94 & B6 & 20 & C6 & E9 & $8 F$ & 44 & D3 & 40 & $9 F$
\end{tabular} $\begin{array}{llllllllllllllllll}\text { A } & 45 & 83 & 96 & 4 B & 6 B & \text { E5 } & 14 & \text { D7 } & 7 C & \text { CD } & \text { AD OA } & 16 & \text { A2 } & 52 & 55\end{array}$ \begin{tabular}{l|l|l|l|l|l|l|l|l|l|l|l|l|l|l|l|l|l} 
B & 71 & 05 & 49 & FO & 25 & 56 & 54 & OE & 21 & $8 \mathrm{C}$ & 24 & 29 & 73 & $\mathrm{~EB}$ & $\mathrm{~B} 3$ & $\mathrm{FE}$
\end{tabular} \begin{tabular}{l|l|l|l|l|l|l|l|l|l|l|l|l|l|l|l|l} 
C & 82 & BB & 78 & C2 & $7 E$ & $9 A$ & $7 D$ & 69 & $2 A$ & 66 & 07 & 76 & 36 & 23 & CA F1
\end{tabular} \begin{tabular}{l|l|l|l|lllllllllllllll} 
D & 12 & $6 F$ & $B 2$ & DA & $7 B$ & 63 & F3 & 10 & FF & $3 A$ & $7 F$ & D1 & DD CF & 64 & 22
\end{tabular}

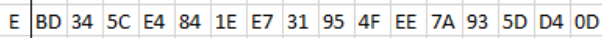
\begin{tabular}{llllllllllllllllllll} 
& $\mathrm{F}$ & 30 & $8 \mathrm{D}$ & $6 \mathrm{E}$ & $8 \mathrm{~B}$ & $3 \mathrm{E}$ & $4 \mathrm{C}$ & $\mathrm{CO}$ & 38 & $5 \mathrm{E}$ & $5 \mathrm{~A}$ & $\mathrm{ED}$ & $\mathrm{B} 5$ & $\mathrm{OB}$ & $\mathrm{CB}$ & 51 & $\mathrm{~A} 1$ \\
\hline
\end{tabular} 
Table 4.6: S-box and Inverse S-box for $x^{8}+x^{6}+x^{4}+x^{3}+x^{2}+x+1$

\begin{tabular}{l|llllllllllllllllll}
0 & 63 & $7 C$ & AA & ED & C1 & E6 & 24 & 88 & 32 & 3 A & A1 & $3 F$ & 86 & 33 & 96 & 64
\end{tabular}

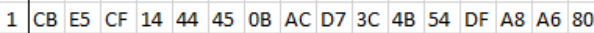

\begin{tabular}{lllllllllllllll|l|l|l|l}
2 & 37 & B9 & 20 & A5 & 73 & BC & D8 & $5 E$ & F0 & $1 D$ & 70 & A9 & 11 & OA & 84 & $2 D$
\end{tabular}

\begin{tabular}{l|l|l|l|l|l|l|l|l|l|l|l|l|l|l|l|l}
3 & $7 F$ & A2 & $8 A$ & 65 & 31 & $4 E$ & F8 & 99 & $7 B$ & D9 & CO & 09 & 81 & 29 & 92 & FA
\end{tabular}

\begin{tabular}{ll|lllllllllllllllll}
4 & OF & EB & 48 & 69 & C2 & 41 & 00 & DE & $6 B$ & B8 & $8 \mathrm{C}$ & $8 \mathrm{E}$ & BE & BA & FD & $4 D$
\end{tabular}

55 EC BF 5 5C A7

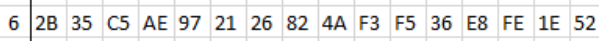

$\begin{array}{lllllllllllllllllll}7 & 6 \mathrm{~F} & 59 & 3 \mathrm{E} & 3 \mathrm{~B} & \mathrm{~B} 2 & 03 & 10 & \mathrm{BB} & 12 & 2 \mathrm{E} & 46 & \mathrm{~B} 6 & 9 \mathrm{~B} & 25 & \mathrm{Eg} & 27\end{array}$

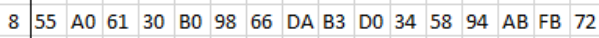

$\begin{array}{lllllllllllllllllll}9 & 67 & \mathrm{EF} & \mathrm{C} 8 & 75 & \mathrm{D} 2 & 2 \mathrm{~F} & \mathrm{D} 3 & 17 & 8 \mathrm{D} & \mathrm{D} 4 & \mathrm{C} 9 & \mathrm{CE} & 2 \mathrm{C} & \mathrm{E} 7 & 74 & 43\end{array}$

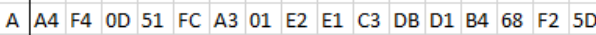

\begin{tabular}{llllll|l|l|l|l|l|l|l|l|l|l}
$B$ & DC F7 & B7 & 16 & $1 A$ & 39 & E3 & $6 C$ & FF & $3 D$ & F6 & 13 & 95 & 50 & EE & $5 A$
\end{tabular}

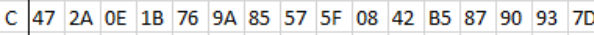

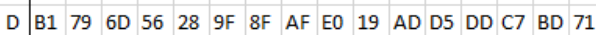

$\begin{array}{lllllllllllllllllll}\text { E } & 23 & 6 \mathrm{~A} & 38 & 0 \mathrm{C} & 8 \mathrm{~B} & 77 & 4 \mathrm{~F} & 7 \mathrm{~A} & \mathrm{CD} & 7 \mathrm{E} & 15 & 04 & 9 \mathrm{C} & 18 & 49 & \mathrm{E} 4\end{array}$

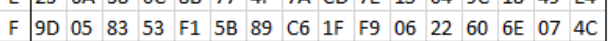

\begin{tabular}{|c|c|c|c|c|c|c|c|c|c|c|c|c|c|c|c|c|}
\hline & & & & & & & & & & & & & & & & \\
\hline & & & $\mathrm{E}$ & & EB & & & & 9 & $B$ & D & 16 & E3 & & & \\
\hline & & & & & & & & & & & & & & & & \\
\hline & & & & & & & & & & & & & & & & \\
\hline & & & & & & & & & & & & 73 & & & & \\
\hline & & & & & & & & & & & & & & & & \\
\hline & & & & & & 0 & & & $8 B$ & , & 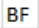 & F5 & 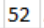 & & & \\
\hline & & & & & & & & & & & & & & & & \\
\hline & & & & & & & & 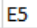 & $F$ & 1 & , & 3 & 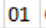 & ( & & \\
\hline & & & & & & & & & & & & & & & & \\
\hline & & & & & & & & 4 & 85 & 37 & C & & & & & \\
\hline & & & & & & & & & & & & 8 & & & & \\
\hline & & & & & & & & & 49 & & & & & & & \\
\hline & & & & & & & & & 9 & A & 3 & 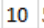 & & $\mathrm{E}$ & & \\
\hline & & & & & & & & & & & & & & & & \\
\hline & & & & & & & & D & $6 C$ & 7 & 54 & 41 & 5 & 03 & & \\
\hline & & & & & & & & & & & & & & & & \\
\hline
\end{tabular}

Table 4.7: S-box and Inverse S-box for $x^{8}+x^{6}+x^{5}+x+1$

\begin{tabular}{|l|l|l|l|l|l|l|l|l|l|l|l|l|l|l|l|l|} 
& 0 & 1 & 2 & 3 & 4 & 5 & 6 & 7 & 8 & 9 & A & B & C & D & E & F \\
\hline
\end{tabular}

\begin{tabular}{l|llllllllllllllllllll}
\hline 0 & 63 & $7 C$ & E1 & 60 & $2 F$ & 62 & E2 & 68 & 48 & $6 C$ & E3 & 34 & AE & 29 & E6 & 95
\end{tabular}

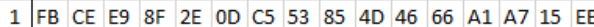

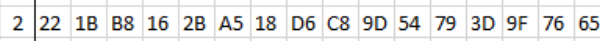

$\begin{array}{lllllllllllllllllll}3 & 1 \mathrm{D} & 17 & 74 & \text { OE } & \text { F1 } & 31 & \text { EC } & 51 & \text { OF } & 8 \mathrm{C} & 01 & \text { AB } & 58 & 43 & 2 \mathrm{~A} & \mathrm{~B} 0\end{array}$

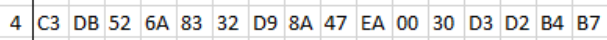

$\begin{array}{llllllllllllllllll}5 & \text { B6 } & 81 & 11 & 4 F & \text { F8 } & \text { B1 } & 6 E & 02 & 41 & 7 B & 10 & 96 & \text { E4 } & 8 D & 6 D & 5 B\end{array}$

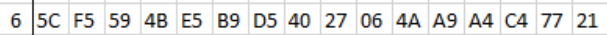

\begin{tabular}{l|lllllll|l|l|l|l|l|l|l|l|l|l}
7 & 55 & $9 \mathrm{E}$ & 99 & 56 & $5 \mathrm{~F}$ & 05 & $0 \mathrm{~A}$ & 37 & $\mathrm{~F} 3$ & $2 \mathrm{C}$ & $7 \mathrm{E}$ & $\mathrm{CO}$ & $\mathrm{C} 7$ & $9 \mathrm{C}$ & 87 & 14
\end{tabular}

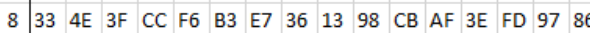

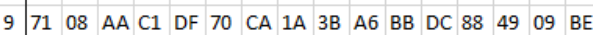

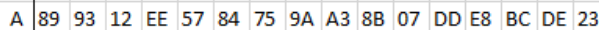

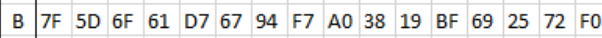

$\begin{array}{lllllllllllllllllll}\text { C } & \text { FC } & \text { BA } & 28 & \text { F4 } & 73 & 9 B & 7 A & 3 A & 20 & \text { CD } & 03 & \text { A2 } & 35 & \text { D4 } & \text { FF } & 5 A\end{array}$

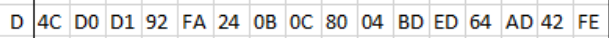

$\begin{array}{llllllllllllllllllll}\text { E } & 78 & 82 & 90 & \text { AC } & 1 E & \text { A8 } & \text { F9 } & \text { C6 } & 7 D & 1 F & 50 & 6 B & \text { DA CF } & 44 & \text { EF }\end{array}$

\begin{tabular}{lllllllllllllllllll} 
F & 26 & 39 & C9 & C2 & E0 & $8 E$ & $B 2$ & $5 E$ & $3 C$ & $B 5$ & 91 & 45 & $1 C$ & F2 & D8 & $2 D$ \\
\hline
\end{tabular} \begin{tabular}{|l|l|l|l|l|l|l|l|l|l|l|l|l|l|l|l|l|}
\hline & 0 & 1 & 2 & 3 & 4 & 5 & 6 & 7 & 8 & 9 & A & B & C & D & E & F \\
\hline
\end{tabular} \begin{tabular}{l|lllll|l|l|l|l|l|l|l|l|l|l|l|l}
0 & 4A & $3 A$ & 57 & CA & D9 & 75 & 69 & AA & 91 & $9 E$ & 76 & D6 & D7 & 15 & 33 & 38
\end{tabular} \begin{tabular}{l|lllllllllllllllllll}
1 & $5 A$ & 52 & A2 & 88 & $7 F$ & $1 E$ & 23 & 31 & 26 & BA & 97 & 21 & FC & 30 & E4 & E9
\end{tabular}

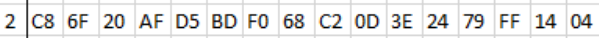
$\begin{array}{llllllllllllllllllll}3 & 4 \mathrm{~B} & 35 & 45 & 80 & \text { OB } & \text { CC } & 87 & 77 & \text { B9 } & \text { F1 } & \text { C7 } & 98 & \text { F } & \text { 2C } & 8 \mathrm{C} & 82\end{array}$

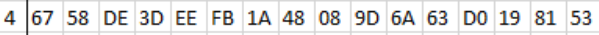
$\begin{array}{llllllllllllllllll}5 & \text { EA } & 37 & 42 & 17 & 2 A & 70 & 73 & \text { A4 } & 3 \mathrm{C} & 62 & \text { CF } & 5 F & 60 & \text { B1 } & \text { F7 } & 74\end{array}$ \begin{tabular}{llllllll|l|l|l|l|l|l|l|l|l}
6 & 03 & B3 & 05 & 00 & DC & $2 F$ & $1 B$ & B5 & 07 & BC & 43 & EB & 09 & $5 E$ & 56 & B2
\end{tabular} $\begin{array}{llllllllllllllllll}7 & 95 & 90 & \text { BE } & \text { C4 } & 32 & \text { A6 } & 2 \mathrm{E} & 6 \mathrm{E} & \mathrm{E} 0 & 2 \mathrm{~B} & \mathrm{C} 6 & 59 & 01 & \text { E8 } & 7 \mathrm{~A} & \mathrm{~B} 0\end{array}$ \begin{tabular}{l|llllllllllllllllll}
8 & D8 & 51 & E1 & 44 & A5 & 18 & $8 \mathrm{~F}$ & $7 \mathrm{E}$ & $9 \mathrm{C}$ & A0 & 47 & A9 & 39 & $5 \mathrm{D}$ & $\mathrm{F} 5$ & 13
\end{tabular} 9 E2 \begin{tabular}{l|llllllll|l|l|l|l|l|l|l|l} 
A & B8 & $1 \mathrm{C}$ & $\mathrm{CB}$ & $\mathrm{A} 8$ & $6 \mathrm{C}$ & 25 & 99 & $1 \mathrm{D}$ & $\mathrm{E} 5$ & $6 \mathrm{~B}$ & 92 & $3 \mathrm{~B}$ & $\mathrm{E} 3$ & $\mathrm{DD}$ & $0 \mathrm{C}$ & $8 \mathrm{~B}$
\end{tabular}

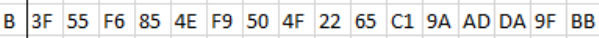
$\begin{array}{llllllllllllllllllll}\text { C } & 7 B & 93 & \text { F3 } & 40 & 6 D & 16 & \text { E7 } & 7 C & 28 & \text { F2 } & 96 & 8 A & 83 & \text { C9 } & 11 & \text { ED }\end{array}$

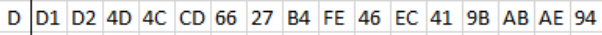
E $\begin{array}{llllllllllllllllll} & 02 & 06 & \text { OA } & 5 C & 64 & 0 E & 86 & \text { AC } & 12 & 49 & \text { 1F } & 36 & \text { DB } & \text { A3 } & \text { EF }\end{array}$ \begin{tabular}{lllllllllllllllllll}
$\mathrm{F}$ & $\mathrm{BF}$ & 34 & $\mathrm{FD}$ & 78 & $\mathrm{C} 3$ & 61 & 84 & $\mathrm{~B} 7$ & 54 & $\mathrm{E} 6$ & $\mathrm{D} 4$ & 10 & $\mathrm{C} 0$ & $8 \mathrm{D}$ & $\mathrm{DF}$ & $\mathrm{CE}$ \\
\hline
\end{tabular}

Table 4.8: S-box and Inverse S-box for $x^{8}+x^{6}+x^{5}+x^{2}+1$

\begin{tabular}{|c|c|c|c|c|c|c|c|c|c|c|c|c|c|c|c|c|}
\hline & 0 & 1 & 2 & 3 & 4 & 5 & 6 & 7 & 8 & 9 & A & B & C & D & $\mathrm{E}$ & $\mathrm{F}$ \\
\hline & 63 & 7C & $\mathrm{CO}$ & $5 E$ & B2 & 40 & FD & $5 A$ & A7 & 74 & $\mathrm{DE}$ & BB & $2 C$ & D6 & D3 & B5 \\
\hline 1 & 01 & 19 & C4 & EO & 91 & DD & 23 & 38 & E8 & B6 & 95 & $6 \mathrm{E}$ & 3B & D4 & 08 & ED \\
\hline 2 & $7 E$ & E6 & 72 & 9A & 9C & 51 & $8 \mathrm{E}$ & F1 & $1 \mathrm{~A}$ & $8 \mathrm{C}$ & 10 & 18 & 43 & AF & CE & 86 \\
\hline 8 & $8 \mathrm{~A}$ & 4B & 89 & 82 & 34 & $2 A$ & C9 & E7 & $4 \mathrm{~F}$ & 9D & 94 & 98 & FA & $3 \mathrm{D}$ & 24 & 09 \\
\hline 4 & C1 & BA & A1 & $6 \mathrm{D}$ & EB & 81 & $9 \mathrm{~F}$ & 61 & Bo & 04 & 56 & FE & B9 & 02 & 06 & 92 \\
\hline P & F3 & 2D & B8 & 84 & F6 & C3 & F2 & 28 & $5 F$ & $1 \mathrm{~F}$ & 05 & 67 & 99 & $3 \mathrm{~A}$ & BD & $6 \mathrm{C}$ \\
\hline 6 & 97 & $2 F$ & 5B & D7 & 16 & CB & 93 & 78 & E4 & DA & C7 & F4 & 36 & E9 & 21 & 45 \\
\hline 7 & 75 & F7 & 30 & 47 & B4 & 7B & $9 \mathrm{E}$ & D8 & 83 & E3 & 60 & $\mathrm{OE}$ & EC & $4 \mathrm{~A}$ & 7A & 39 \\
\hline 8 & 32 & 55 & A3 & 52 & $2 E$ & 77 & 48 & $4 \mathrm{C}$ & OB & 14 & 12 & $2 \mathrm{~B}$ & 31 & $8 \mathrm{~F}$ & 62 & E1 \\
\hline 9 & A6 & $3 F$ & D0 & 27 & F9 & 80 & $A D$ & 00 & 22 & F5 & $\mathrm{FF}$ & AC & D1 & 25 & 9B & $6 \mathrm{~B}$ \\
\hline $\mathrm{A}$ & 07 & 46 & 68 & 90 & A2 & F0 & BC & $\mathrm{CD}$ & A9 & $6 \mathrm{~A}$ & 33 & F8 & 87 & B7 & C6 & $\mathrm{CC}$ \\
\hline B & 7D & 49 & 5D & 41 & 50 & 96 & 4D & D2 & $1 \mathrm{E}$ & $8 \mathrm{D}$ & CF & $3 \mathrm{C}$ & OC & 26 & C8 & 79 \\
\hline$c$ & 35 & OD & 69 & $1 \mathrm{C}$ & 53 & $\mathrm{AE}$ & 15 & 03 & D9 & AA & 37 & 17 & $1 \mathrm{~B}$ & $5 \mathrm{C}$ & EE & $\mathrm{C} 2$ \\
\hline U & A0 & FB & BF & C5 & 1D & 20 & A8 & $8 \mathrm{~B}$ & E5 & EA & OA & 76 & 42 & DF & 70 & 11 \\
\hline$E$ & 44 & 66 & 29 & DC & CA & 64 & 71 & 54 & 88 & 58 & $6 \mathrm{~F}$ & $7 F$ & B1 & 57 & $\mathrm{BE}$ & 85 \\
\hline & 13 & 55 & $\mathrm{~F}$ & 59 & E2 & $3 \mathrm{E}$ & D5 & $\mathrm{AB}$ & A4 & A5 & DB & B3 & EF & 73 & $4 \mathrm{E}$ & FC \\
\hline
\end{tabular}

\begin{tabular}{llllllllllllllllllllll} 
& 0 & 1 & 2 & 3 & 4 & 5 & 6 & 7 & 8 & 9 & A & B & C & D & $E$ & $F$ \\
\hline
\end{tabular} \begin{tabular}{|l|lllllllllllllllllllll}
\hline 0 & 97 & 10 & $4 \mathrm{D}$ & $\mathrm{C} 7$ & 49 & $5 \mathrm{~A}$ & $4 \mathrm{E}$ & A0 & $1 \mathrm{E}$ & $3 \mathrm{~F}$ & $\mathrm{DA}$ & 88 & BC & C1 & $7 \mathrm{~B}$ & F2 \\
\hline
\end{tabular} \begin{tabular}{llllllllll|l|l|l|l|l|l|l|l}
1 & $2 A$ & DF & $8 A$ & F0 & 89 & C6 & 64 & CB & $2 B$ & 11 & 28 & CC & C3 & D4 & B8 & 59
\end{tabular} $\begin{array}{llllllllllllllllll}2 & D 5 & 6 E & 98 & 16 & 3 E & 9 D & B D & 93 & 57 & \text { E2 } & 35 & 8 B & 0 C & 51 & 84 & 61\end{array}$ \begin{tabular}{l|l|l|l|l|l|l|l|l|l|l|l|l|l|l|l|l}
3 & 72 & $8 \mathrm{C}$ & 80 & AA & 34 & C0 & $6 C$ & CA & 17 & $7 F$ & $5 D$ & $1 C$ & BB & $3 D$ & F5 & 91
\end{tabular}

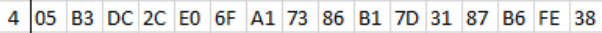
\begin{tabular}{l|l|l|l|l|l|l|l|l|l|l|l|l|l|l|l}
5 & B4 & 25 & 83 & C4 & E7 & 81 & $4 A$ & ED E9 & F3 & 07 & 62 & CD & B2 & 03 & 58
\end{tabular} 6 7A 47 8E 00 E5 F1 E1 5B A2 C2 A9 9F 5F 43 1B EA

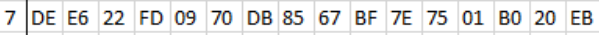
$\begin{array}{lllllllllllllllll}8 & 95 & 45 & 33 & 78 & 53 & \text { EF } & 2 F & \text { AC E8 } & 32 & 30 & \text { D7 } & 29 & \text { B9 } & 26 & 8 D\end{array}$ \begin{tabular}{l|llllllllllllll|l|l|l|l|}
9 & A3 & 14 & $4 \mathrm{~F}$ & 66 & $3 \mathrm{~A}$ & $1 \mathrm{~A}$ & $\mathrm{~B} 5$ & 60 & $3 \mathrm{~B}$ & $5 \mathrm{C}$ & 23 & $9 \mathrm{E}$ & 24 & 39 & 76 & 46
\end{tabular} $\begin{array}{llllllllllllllllll}A & \text { D0 } & 42 & \text { A4 } & 82 & \text { F8 } & \text { F9 } & 90 & 08 & \text { D6 } & \text { A8 } & \text { C9 } & \text { F7 } & 9 B & 96 & \text { C5 } & \text { 2D }\end{array}$ B 48 EC 04 FB 74 OF 19 AD 52 4C 41 OB A6 5 EE EE D2

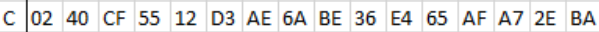
$\begin{array}{lllllllllllllllllll}D & 92 & 9 C & B 7 & \text { OE } & 1 D & \text { F6 } & \text { OD } & 63 & 77 & \text { C8 } & 69 & \text { FA } & \text { E3 } & 15 & \text { OA } & \text { DD }\end{array}$

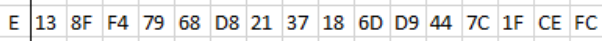
\begin{tabular}{l|llllllllllllllll} 
F & A5 & 27 & 56 & 50 & $6 B$ & 99 & 54 & 71 & AB & 94 & $3 \mathrm{C}$ & D1 & FF & 06 & $4 B$ & $9 A$
\end{tabular} 
Table 4.9: S-box and Inverse S-box for $x^{8}+x^{6}+x^{5}+x^{3}+1$

\begin{tabular}{|c|c|c|c|c|c|c|c|c|c|c|c|c|c|c|c|c|}
\hline & 0 & 1 & 2 & 3 & 4 & 5 & 6 & 7 & 8 & 9 & A & B & C & D & $E$ & $\mathrm{~F}$ \\
\hline 0 & 63 & $7 \mathrm{C}$ & 82 & 22 & 93 & $5 C$ & C3 & $4 \mathrm{E}$ & $1 \mathrm{~B}$ & 78 & $\mathrm{FC}$ & 73 & 33 & $\mathrm{CC}$ & F5 & 89 \\
\hline 1 & 31 & $8 \mathrm{E}$ & $\mathrm{EE}$ & 81 & $\mathrm{AC}$ & $9 \mathrm{E}$ & $6 \mathrm{~B}$ & F9 & 4B & C5 & DA & $6 \mathrm{~A}$ & 28 & 27 & 16 & 38 \\
\hline 2 & $4 A$ & E1 & FB & $4 \mathrm{D}$ & A5 & $6 C$ & 12 & A0 & 84 & 04 & F3 & 01 & 67 & $9 \mathrm{~B}$ & 40 & $1 \mathrm{~A}$ \\
\hline 3 & 19 & ED & $5 \mathrm{E}$ & C7 & BF & C2 & E7 & D3 & C6 & EA & $2 \mathrm{~F}$ & 58 & D9 & 96 & CE & 83 \\
\hline 4 & 99 & 77 & $4 C$ & D7 & 41 & 50 & 74 & CF & 00 & $8 C$ & $8 \mathrm{~A}$ & AF & B5 & C1 & EC & 59 \\
\hline 5 & $\mathrm{FE}$ & 80 & DO & E6 & 45 & B4 & $3 C$ & E5 & $\mathrm{OF}$ & B8 & $1 \mathrm{~F}$ & 43 & $9 \mathrm{C}$ & CB & B1 & 48 \\
\hline 6 & 30 & A8 & 24 & E2 & FD & 79 & $5 \mathrm{~F}$ & 25 & OD & $\mathrm{DD}$ & B3 & F1 & 21 & 51 & 3B & 66 \\
\hline 7 & DF & $\mathrm{FF}$ & $\mathrm{CP}$ & 61 & $2 \mathrm{~B}$ & 11 & 90 & $7 F$ & $3 \mathrm{E}$ & DC & F7 & $0 A$ & DB & F6 & 13 & $O C$ \\
\hline 8 & $1 \mathrm{E}$ & 46 & 07 & 69 & $\mathrm{~F} 4$ & 60 & 57 & EF & $1 \mathrm{C}$ & $6 \mathrm{D}$ & 94 & BD & 86 & BB & 5B & 87 \\
\hline 9 & $B C$ & 26 & FA & 17 & 97 & D6 & 05 & $3 \mathrm{~A}$ & 08 & 23 & 32 & E9 & A4 & 68 & 10 & B2 \\
\hline A & $A D$ & A7 & 92 & 91 & BA & E3 & A1 & E4 & 70 & 15 & 88 & $7 E$ & A2 & $2 \mathrm{~A}$ & 20 & $\mathrm{DE}$ \\
\hline B & 55 & $9 \mathrm{~F}$ & EO & B6 & 5D & $3 D$ & 1D & 39 & F2 & 71 & 37 & 09 & 64 & A6 & 98 & 56 \\
\hline C & CA & $0 \mathrm{E}$ & E8 & $6 \mathrm{~F}$ & $\mathrm{AE}$ & F0 & $C D$ & 18 & $2 \mathrm{C}$ & F8 & $6 \mathrm{E}$ & 06 & $7 \mathrm{D}$ & 95 & $2 \mathrm{E}$ & $8 \mathrm{D}$ \\
\hline D & 54 & 75 & 52 & BE & 65 & 7B & 44 & 49 & 42 & $9 \mathrm{D}$ & 14 & 72 & $4 \mathrm{~F}$ & D8 & $8 \mathrm{~F}$ & EB \\
\hline$E$ & 53 & 85 & $2 \mathrm{D}$ & $\mathrm{C} 4$ & 36 & $5 \mathrm{~A}$ & 62 & BO & 47 & B7 & 34 & D5 & $9 \mathrm{~A}$ & C8 & 03 & $\mathrm{OB}$ \\
\hline & 13 & 02 & D2 & 76 & 29 & 1 & B9 & $7 \mathrm{~A}$ & $3 \mathrm{~F}$ & AA & A9 & $\mathrm{CO}$ & 35 & $8 \mathrm{~B}$ & D4 & D1 \\
\hline
\end{tabular}

\begin{tabular}{|c|c|c|c|c|c|c|c|c|c|c|c|c|c|c|c|c|}
\hline & 0 & 1 & 2 & 3 & 4 & 5 & 6 & 1 & 8 & 9 & A & B & C & D & $\mathrm{E}$ & \\
\hline 0 & 48 & $2 B$ & F1 & $\mathrm{EE}$ & 29 & 96 & CB & 82 & 98 & BB & $7 B$ & EF & $7 F$ & 68 & C1 & 58 \\
\hline 1 & $9 \mathrm{E}$ & 75 & 26 & $7 E$ & DA & A9 & $1 \mathrm{E}$ & 93 & C7 & 30 & $2 \mathrm{~F}$ & 08 & 88 & B6 & 80 & $5 A$ \\
\hline 2 & $\mathrm{AE}$ & $6 \mathrm{C}$ & 03 & 99 & 62 & 67 & 91 & 1D & $1 \mathrm{C}$ & F4 & $A D$ & 74 & C8 & E2 & $\mathrm{CE}$ & $3 A$ \\
\hline 3 & 60 & 10 & 9A & OC & EA & FC & E4 & BA & $1 \mathrm{~F}$ & B7 & 97 & $6 \mathrm{E}$ & 56 & B5 & 78 & F8 \\
\hline 4 & $2 E$ & 44 & D8 & $5 B$ & D6 & 54 & 81 & E8 & $5 F$ & D7 & 20 & 18 & 42 & 23 & 07 & DC \\
\hline 5 & 45 & $6 \mathrm{D}$ & D2 & EO & D0 & B0 & BF & 86 & 3B & $4 \mathrm{~F}$ & E5 & $8 \mathrm{E}$ & 05 & B4 & 32 & 66 \\
\hline 6 & 85 & 73 & E6 & 00 & BC & D4 & $6 \mathrm{~F}$ & $2 C$ & $9 D$ & 83 & $1 \mathrm{~B}$ & 16 & 25 & 89 & CA & C3 \\
\hline 7 & A8 & B9 & DB & OB & 46 & D1 & F3 & 41 & 09 & 65 & F7 & D5 & 01 & CC & AB & 77 \\
\hline 8 & 51 & 13 & 02 & $3 F$ & 28 & E1 & $8 \mathrm{C}$ & $8 \mathrm{~F}$ & AA & OF & $4 \mathrm{~A}$ & FD & 49 & $\mathrm{CF}$ & 11 & DE \\
\hline 9 & 76 & A3 & A2 & 04 & $8 \mathrm{~A}$ & $C D$ & $3 D$ & 94 & BE & 40 & EC & $2 \mathrm{D}$ & $5 C$ & D9 & 15 & B1 \\
\hline A & 27 & A6 & $A C$ & FO & 9C & 24 & BD & A1 & 61 & FA & F9 & F5 & 14 & A0 & C4 & 4B \\
\hline B & E7 & $5 E$ & $9 \mathrm{~F}$ & $6 \mathrm{~A}$ & 55 & $4 C$ & B3 & E9 & 59 & F6 & A4 & $8 D$ & 90 & $8 B$ & D3 & 34 \\
\hline C & FB & 4D & 35 & 06 & E3 & 19 & 38 & 33 & ED & 72 & $\mathrm{CO}$ & 5D & OD & C6 & $3 \mathrm{E}$ & 47 \\
\hline D & 52 & FF & F2 & 37 & $\mathrm{FE}$ & EB & 95 & 43 & DD & $3 C$ & $1 \mathrm{~A}$ & 7C & 79 & 69 & $\mathrm{AF}$ & 70 \\
\hline$E$ & B2 & 21 & 63 & A5 & A7 & 5 & 53 & 36 & $\mathrm{C} 2$ & 3 & 39 & DF & $4 \mathrm{E}$ & 31 & 12 & 87 \\
\hline & & & & & & & & & & & & & & & & \\
\hline
\end{tabular}

Table 4.10: S-box and Inverse S-box for $x^{8}+x^{6}+x^{5}+x^{4}+1$

\begin{tabular}{|c|c|c|c|c|c|c|c|c|c|c|c|c|c|c|c|c|}
\hline & 0 & 1 & 2 & 3 & 4 & 5 & 6 & 7 & 8 & 9 & A & B & C & $D$ & $E$ & $F$ \\
\hline 0 & 63 & 7C & 06 & DA & D1 & B8 & $\mathrm{BF}$ & $\mathrm{AE}$ & $3 A$ & 94 & 64 & 35 & OD & 71 & 85 & D5 \\
\hline 1 & CF & $B D$ & 72 & 74 & $0 A$ & AF & A2 & 92 & 54 & $9 D$ & $6 \mathrm{~A}$ & 82 & FA & 51 & D2 & 43 \\
\hline 2 & DF & 30 & $O C$ & 77 & EB & $5 E$ & 02 & C1 & $3 \mathrm{D}$ & AA & 05 & $7 A$ & 69 & C3 & $9 \mathrm{~B}$ & $8 B$ \\
\hline 3 & F8 & 12 & F6 & 29 & E7 & $6 \mathrm{E}$ & 93 & 61 & 45 & $7 F$ & 90 & F2 & BB & C8 & 99 & D8 \\
\hline 4 & D7 & F5 & CA & D9 & D4 & 86 & 83 & 49 & $\mathrm{CD}$ & 37 & FD & DB & 39 & $5 F$ & 32 & 38 \\
\hline 5 & A6 & 57 & $6 \mathrm{D}$ & B3 & 50 & E9 & EF & 34 & 66 & $2 \mathrm{E}$ & 33 & $7 E$ & $1 \mathrm{~F}$ & A7 & 17 & $2 B$ \\
\hline 6 & 44 & 11 & 31 & $A D$ & A9 & $6 \mathrm{~F}$ & 46 & 65 & 21 & B1 & $\mathrm{OF}$ & E8 & $1 \mathrm{~B}$ & 76 & 62 & $\mathrm{C} 4$ \\
\hline 7 & 70 & $3 B$ & 87 & 53 & $9 \mathrm{~A}$ & C6 & 41 & FC & E5 & 09 & B6 & 24 & $1 \mathrm{E}$ & 27 & BE & 68 \\
\hline 8 & D3 & D6 & 28 & $5 C$ & B7 & ED & $3 \mathrm{E}$ & A0 & 52 & $\mathrm{FE}$ & 7B & BA & 13 & A1 & $9 \mathrm{C}$ & F9 \\
\hline 9 & DE & $\mathrm{C} 2$ & A3 & 18 & $2 C$ & $8 \mathrm{C}$ & $3 F$ & 26 & $4 \mathrm{E}$ & 16 & 7D & E2 & CB & C7 & CE & 00 \\
\hline A & 81 & F7 & 79 & B2 & $8 \mathrm{E}$ & 95 & E1 & 14 & 10 & $9 \mathrm{E}$ & $\mathrm{CC}$ & F3 & 25 & 84 & 22 & 67 \\
\hline B & OB & 4D & $2 F$ & 80 & 4B & 75 & 07 & 23 & 5D & C5 & 01 & $2 \mathrm{D}$ & 59 & $1 \mathrm{~A}$ & 47 & $\mathrm{OE}$ \\
\hline C & Fo & 08 & B0 & $\mathrm{EE}$ & $4 \mathrm{~A}$ & B9 & 04 & DC & EC & $\mathrm{CP}$ & $8 \mathrm{~F}$ & A8 & F1 & 15 & $8 \mathrm{~A}$ & 73 \\
\hline D & 42 & 19 & EO & B4 & 55 & 48 & $4 \mathrm{C}$ & 88 & B5 & 78 & 03 & $1 \mathrm{C}$ & E3 & F4 & $5 A$ & 96 \\
\hline $\mathrm{E}$ & EA & A4 & $4 F$ & 36 & FB & $\mathrm{FF}$ & 91 & $6 C$ & $9 \mathrm{~F}$ & $\mathrm{CO}$ & 5B & 58 & 98 & 97 & $\mathrm{AC}$ & $6 \mathrm{~B}$ \\
\hline & 20 & 40 & $B C$ & 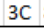 & 89 & 60 & $2 \mathrm{~A}$ & A5 & & 1D & $A B$ & E4 & $8 \mathrm{D}$ & D0 & E6 & 56 \\
\hline
\end{tabular}

\begin{tabular}{|c|c|c|c|c|c|c|c|c|c|c|c|c|c|c|c|c|}
\hline & 0 & 1 & 2 & 3 & 4 & 5 & 6 & 7 & 8 & 9 & A & B & C & D & $E$ & $F$ \\
\hline 0 & $9 \mathrm{~F}$ & BA & 26 & DA & C6 & $2 \mathrm{~A}$ & 02 & B6 & C1 & 79 & 14 & BO & 22 & $O C$ & $B F$ & $6 \mathrm{~A}$ \\
\hline 1 & A8 & 61 & 31 & $8 \mathrm{C}$ & A7 & $C D$ & 99 & $5 \mathrm{E}$ & 93 & D1 & BD & $6 C$ & DB & F9 & $7 \mathrm{C}$ & $5 C$ \\
\hline 2 & F0 & 68 & $\mathrm{AE}$ & B7 & 7B & AC & 97 & 7D & 82 & 33 & F6 & $5 F$ & 94 & BB & 59 & B2 \\
\hline 3 & 21 & 62 & $4 \mathrm{E}$ & $5 A$ & 57 & $\mathrm{OB}$ & E3 & 49 & $4 \mathrm{~F}$ & $4 C$ & 08 & 71 & F3 & 28 & 86 & 96 \\
\hline 4 & F1 & 76 & DO & $1 \mathrm{~F}$ & 60 & 38 & 66 & $\mathrm{BE}$ & D5 & 47 & $\mathrm{C} 4$ & B4 & D6 & B1 & 98 & E2 \\
\hline 5 & 54 & 1D & 88 & 73 & 18 & D4 & $\mathrm{FF}$ & 51 & EB & BC & DE & EA & 83 & B8 & 25 & 4D \\
\hline 6 & F5 & 37 & $6 \mathrm{E}$ & 00 & $O A$ & 67 & 58 & AF & $7 F$ & $2 C$ & $1 \mathrm{~A}$ & EF & E7 & 52 & 35 & 65 \\
\hline 7 & 70 & OD & 12 & CF & 13 & B5 & $6 \mathrm{D}$ & 23 & D9 & A2 & $2 \mathrm{~B}$ & $8 \mathrm{~A}$ & 01 & 9A & 5B & 39 \\
\hline 8 & B3 & A0 & 1B & 46 & $A D$ & $0 \mathrm{E}$ & 45 & 72 & D7 & F4 & $\mathrm{CE}$ & $2 F$ & 95 & $\mathrm{FC}$ & A4 & $C A$ \\
\hline 9 & $3 A$ & E6 & 17 & 36 & 09 & A5 & DF & ED & EC & $3 \mathrm{E}$ & 74 & $2 \mathrm{E}$ & $8 \mathrm{E}$ & 19 & A9 & E8 \\
\hline A & 87 & $8 \mathrm{D}$ & 16 & 92 & E1 & F7 & 50 & 5D & $\mathrm{CB}$ & 64 & 29 & FA & $\mathrm{EE}$ & 63 & 07 & 15 \\
\hline B & C2 & 69 & A3 & 53 & D3 & D8 & $7 A$ & 84 & 05 & C5 & $8 \mathrm{~B}$ & $3 C$ & F2 & 11 & 7E & 06 \\
\hline C & E9 & 27 & 91 & $2 \mathrm{D}$ & $6 \mathrm{~F}$ & B9 & 75 & 9D & 3D & C9 & 42 & $9 \mathrm{C}$ & AA & 48 & $9 \mathrm{E}$ & 10 \\
\hline D & FD & 04 & $1 \mathrm{E}$ & 80 & 44 & $\mathrm{OF}$ & 81 & 40 & $3 \mathrm{~F}$ & 43 & 03 & $4 B$ & C7 & F8 & 90 & 20 \\
\hline$E$ & D2 & A6 & $9 \mathrm{~B}$ & DC & FB & 78 & $\mathrm{FE}$ & 34 & $6 \mathrm{~B}$ & 55 & E0 & 24 & C8 & 85 & C3 & 56 \\
\hline & $\mathrm{CO}$ & $\mathrm{CC}$ & 3B & AB & D & 41 & 32 & A1 & 30 & $8 \mathrm{~F}$ & $1 \mathrm{C}$ & E4 & 77 & $4 \mathrm{~A}$ & 89 & E5 \\
\hline
\end{tabular}

Table 4.11: S-box and Inverse S-box for $x^{8}+x^{7}+x^{2}+x+1$

\begin{tabular}{|c|c|c|c|c|c|c|c|c|c|c|c|c|c|c|c|c|}
\hline & & & & & & & & & & & & & & & & \\
\hline & & & & & & & & & & 3 & 4 & & 9 & $F$ & & \\
\hline & & & & & & & & & & & & & & & & \\
\hline & & & & & & & & & & & & & & & & \\
\hline & & & & & & & & & & & & & & & & \\
\hline & & & & & & & & & & & & & & & & \\
\hline & & & & & & & & & & & & & & & & 68 \\
\hline & & & & & & & & & & & & & & & & \\
\hline & & & & 90 & & $F$ & & 1 & 52 & + & & 43 & $7 E$ & & & 2 \\
\hline & & & & & & & & & & & & & & & & \\
\hline & & & & & & & & B6 & & $2 \mathrm{D}$ & $\mathrm{OE}$ & $8 \mathrm{D}$ & & & & 40 \\
\hline & & & & & & & & & & 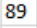 & D6 & & & & & \\
\hline & & & & & & & & & & & & & & & & \\
\hline & & & & & & & & C3 & BO & F & & $\mathrm{E}$ & & & & \\
\hline & & & & & & & & & & & & & & & & \\
\hline & & & & & 5 & & & & & $E$ & D & & B8 & & & \\
\hline & & & & & & & & & & & & & & & & \\
\hline
\end{tabular}

\begin{tabular}{|l|l|l|l|l|l|l|l|l|l|l|l|l|l|l|l|l|}
\hline & 0 & 1 & 2 & 3 & 4 & 5 & 6 & 7 & 8 & 9 & A & B & C & D & E & F \\
\hline
\end{tabular} \begin{tabular}{l|lllllllllllllllllll}
\hline 0 & $7 E$ & FF & $2 E$ & 29 & $2 B$ & $7 A$ & 86 & 72 & A4 & 64 & 02 & F8 & 05 & $1 A$ & $9 A$ & D8 \\
\hline
\end{tabular} \begin{tabular}{l|l|l|l|l|l|l|l|l|l|l|l|l|l|l|l|l}
1 & 27 & 10 & 13 & A3 & E0 & 83 & D5 & 16 & 85 & 76 & 48 & OE & 6 F & F0 & 93 & A2
\end{tabular}

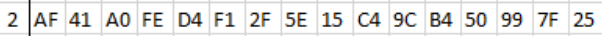
3 CF 04 95 09 FC 5C D1 DD 8D 90 91 1 1D 4 4E 26 5A 53 \begin{tabular}{lll|l|l|l|l|l|l|l|l|l|l|l|l|l}
4 & 70 & $1 F$ & 82 & $7 B$ & 46 & 33 & OF & EF & DO FA & 08 & 12 & $9 F$ & E5 & A7 & $3 A$
\end{tabular}

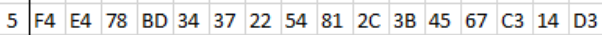
$\begin{array}{lllllllllllllllll}6 & 6 \mathrm{D} & 98 & \mathrm{~B} 7 & 00 & 43 & \mathrm{ED} & 39 & \mathrm{DB} & 5 \mathrm{~F} & \mathrm{DF} & \mathrm{B} 2 & \mathrm{AE} & 17 & 60 & \mathrm{D} 7 & 49\end{array}$ \begin{tabular}{l|l|l|l|l|l|l|l|l|l|l|l|l|l|l|l|l|l}
7 & 66 & 59 & 80 & 57 & $4 D$ & 74 & $6 C$ & BA & $6 E$ & A8 & $9 E$ & E7 & 01 & 21 & $7 C$ & C6
\end{tabular} 8 B6 $\begin{array}{lllllllllllllllll} & \text { CA } & \text { C0 } & 92 & 36 & \text { F7 } & 5 D & 31 & \text { F2 } & \text { A9 } & 42 & \text { 0B } & 40 & \text { 9B } & \text { E8 } & \text { F3 }\end{array}$ \begin{tabular}{l|l|l|l|l|l|l|l|l|l|l|l|l|l|lll}
9 & 73 & 24 & $\mathrm{~EB}$ & 07 & $3 \mathrm{E}$ & $\mathrm{F} 6$ & 63 & 84 & 58 & $8 \mathrm{~A}$ & $\mathrm{E} 6$ & 51 & $4 \mathrm{~F}$ & $\mathrm{FD}$ & $\mathrm{B} 0$ & $4 \mathrm{~B}$
\end{tabular}

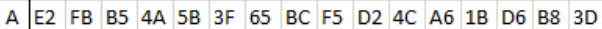

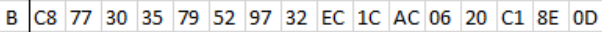

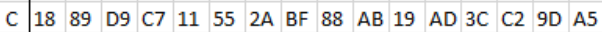
D $\begin{array}{lllllllllllllllllll}56 & 8 B & 03 & \text { CD } & 0 A & \text { B3 } & \text { AA F9 } & 47 & \text { 2D } & \text { C5 } & \text { EA DE A1 } & \text { E1 } & 75\end{array}$ \begin{tabular}{ll|l|l|l|l|l|l|l|l|l|l|l|l|l|l|l|l}
\hline E & 23 & CB & DA & E9 & 71 & 94 & BE & E3 & 38 & OC & CC & 87 & EE & $6 A$ & DC & BB
\end{tabular} \begin{tabular}{lllllllllllllllllll}
$\mathrm{F}$ & $8 \mathrm{C}$ & $1 \mathrm{E}$ & $\mathrm{B} 1$ & 61 & $\mathrm{~B} 9$ & $\mathrm{C} 9$ & 69 & 62 & 68 & 44 & $7 \mathrm{D}$ & 96 & $\mathrm{CE}$ & 28 & $6 \mathrm{~B}$ & $8 \mathrm{~F}$ \\
\hline
\end{tabular} 
Table 4.12: S-box and Inverse S-box for $x^{8}+x^{7}+x^{3}+x^{2}+1$

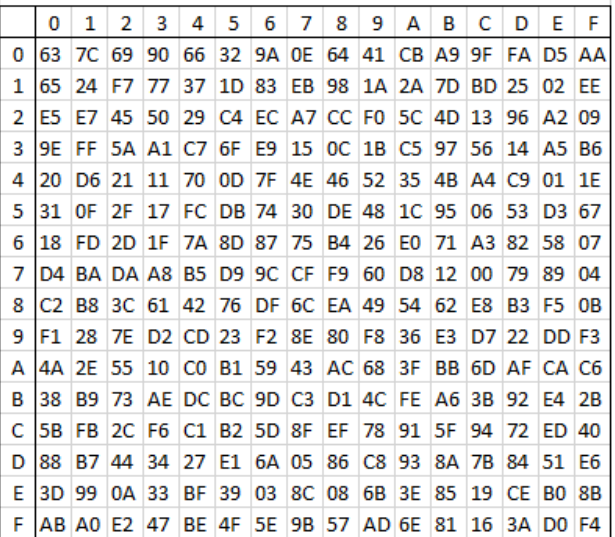

\begin{tabular}{|c|c|c|c|c|c|c|c|c|c|c|c|c|c|c|c|c|}
\hline & 0 & 1 & 2 & 3 & & & & & & & & & & & & \\
\hline 0 & 7C & $4 \mathrm{E}$ & $1 \mathrm{E}$ & E6 & $7 F$ & D7 & $5 \mathrm{C}$ & $6 \mathrm{~F}$ & E8 & $2 \mathrm{~F}$ & E2 & $8 \mathrm{~F}$ & 38 & 45 & 07 & 51 \\
\hline & 3 & 43 & 7B & $2 \mathrm{C}$ & 3D & 37 & $\mathrm{FC}$ & 53 & 60 & EC & 19 & 39 & $5 \mathrm{~A}$ & 15 & $4 \mathrm{~F}$ & 63 \\
\hline 2 & 0 & 42 & $9 \mathrm{D}$ & 95 & 11 & $1 D$ & 69 & D4 & 1 & 24 & 1A & BF & $\mathrm{C} 2$ & 62 & A1 & 52 \\
\hline 3 & 57 & 50 & 05 & E3 & D3 & $4 \mathrm{~A}$ & $9 \mathrm{~A}$ & 14 & BO & E5 & FD & $B C$ & 82 & EO & EA & A \\
\hline 4 & CF & 09 & 84 & A7 & D2 & 22 & 48 & F3 & 59 & 89 & AO & $4 B$ & B9 & $2 \mathrm{~B}$ & 47 & F5 \\
\hline 5 & 23 & $\mathrm{DE}$ & 49 & 5D & $8 \mathrm{~A}$ & A2 & $3 C$ & F8 & $6 \mathrm{E}$ & 6 & 32 & $\mathrm{CO}$ & $2 \mathrm{~A}$ & C6 & F6 & $\mathrm{CB}$ \\
\hline 6 & 79 & 83 & $8 \mathrm{~B}$ & 00 & 08 & 10 & 04 & $5 F$ & A9 & 02 & D6 & E9 & 87 & A & 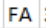 & 35 \\
\hline 7 & 44 & $6 \mathrm{~B}$ & $C D$ & B2 & 56 & 67 & 85 & 13 & 9 & 7 & 64 & DC & 0 & $1 \mathrm{~B}$ & 92 & 46 \\
\hline 8 & 98 & FB & $6 \mathrm{D}$ & 16 & $\mathrm{DD}$ & EB & D8 & 66 & D0 & $7 \mathrm{E}$ & DB & EF & E7 & 65 & 97 & C7 \\
\hline & 03 & CA & $B D$ & DA & $\mathrm{CC}$ & $5 B$ & $2 D$ & 3 & & E1 & 06 & F7 & 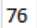 & B & 30 & $O C$ \\
\hline A & $\mathrm{F} 1$ & 33 & $2 \mathrm{E}$ & $6 C$ & $4 C$ & $3 \mathrm{E}$ & BB & 27 & 73 & $\mathrm{OB}$ & $\mathrm{OF}$ & FO & A8 & F9 & B3 & $A D$ \\
\hline & $\mathrm{EE}$ & A5 & C5 & $8 \mathrm{D}$ & 68 & 74 & $3 \mathrm{~F}$ & D1 & 81 & 1 & 71 & $A B$ & B5 & 1C & F4 & E4 \\
\hline & A4 & $\mathrm{C} 4$ & 80 & B7 & 25 & $3 A$ & $\mathrm{AF}$ & 34 & D9 & $4 D$ & $\mathrm{AE}$ & $O A$ & 2 & 94 & ED & 77 \\
\hline D & $\mathrm{FE}$ & B8 & 93 & $5 \mathrm{E}$ & 70 & $\mathrm{OE}$ & 41 & $9 \mathrm{C}$ & $7 \mathrm{~A}$ & 75 & 72 & 55 & B4 & $9 \mathrm{E}$ & 58 & 86 \\
\hline & $6 \mathrm{~A}$ & D5 & F2 & $9 \mathrm{~B}$ & $\mathrm{BE}$ & 20 & DF & 21 & $8 \mathrm{C}$ & 3 & 88 & 17 & 26 & $\mathrm{CE}$ & $1 \mathrm{~F}$ & C \\
\hline & 29 & 90 & 96 & $9 \mathrm{~F}$ & $\mathrm{FF}$ & $8 \mathrm{E}$ & C3 & 12 & 99 & 88 & $O D$ & $c$ & & 61 & BA & 1 \\
\hline
\end{tabular}

Table 4.13: S-box and Inverse S-box for $x^{8}+x^{7}+x^{5}+x^{3}+1$

\begin{tabular}{|c|c|c|c|c|c|c|c|c|c|c|c|c|c|c|c|c|}
\hline & 0 & 1 & 2 & 3 & 4 & 5 & 6 & 7 & 8 & 9 & A & B & C & $D$ & $E$ & $F$ \\
\hline ] & 63 & 7C & A6 & E5 & 81 & $6 \mathrm{D}$ & 20 & 83 & 12 & BO & $2 \mathrm{E}$ & E6 & $\mathrm{C} 2$ & 79 & 13 & 15 \\
\hline 1 & 91 & 3D & $\mathrm{CO}$ & $6 \mathrm{~A}$ & $8 \mathrm{~F}$ & A9 & A1 & 19 & B3 & DF & $6 \mathrm{E}$ & $5 \mathrm{E}$ & 11 & $6 C$ & 58 & 27 \\
\hline 2 & $1 \mathrm{~A}$ & A0 & 06 & DO & B2 & A3 & E7 & 66 & $5 \mathrm{~F}$ & $\mathrm{FF}$ & $4 \mathrm{C}$ & 7B & 48 & FB & 14 & D4 \\
\hline 8 & 41 & 54 & 77 & DA & AF & 9B & FD & C3 & 10 & 39 & AE & F7 & B4 & 01 & $\mathrm{OB}$ & 25 \\
\hline 4 & 95 & D2 & C8 & CA & D1 & 34 & BA & 31 & $\mathrm{C} 1$ & D3 & 49 & 55 & 21 & $9 \mathrm{C}$ & $A B$ & EA \\
\hline 5 & 7D & 56 & $2 \mathrm{D}$ & 02 & F4 & 87 & $6 \mathrm{~F}$ & 07 & BC & $\mathrm{FC}$ & 65 & $\mathrm{FE}$ & D8 & $2 \mathrm{~B}$ & F2 & B8 \\
\hline 6 & 38 & 28 & F8 & DD & 23 & 7E & BF & 80 & 05 & $9 \mathrm{~F}$ & $1 \mathrm{~F}$ & 72 & $2 \mathrm{C}$ & E2 & 33 & $\mathrm{DC}$ \\
\hline 7 & 90 & F1 & 4E & AA & 85 & D7 & 29 & 98 & 88 & B5 & 18 & 17 & 1D & $6 \mathrm{~B}$ & OA & D9 \\
\hline 8 & 52 & 86 & BB & $8 \mathrm{~A}$ & B6 & $\mathrm{EE}$ & B7 & E8 & $3 \mathrm{~A}$ & CB & 82 & A2 & $\mathrm{C5}$ & $4 \mathrm{~F}$ & $4 \mathrm{~A}$ & 62 \\
\hline 9 & 32 & 74 & 3B & 61 & $3 \mathrm{C}$ & 24 & 78 & EO & 42 & B9 & D6 & 44 & $4 \mathrm{D}$ & F3 & ED & Fo \\
\hline $\mathrm{A}$ & 26 & 89 & F9 & A5 & OE & 09 & 99 & $\mathrm{EC}$ & A8 & B1 & 5B & DB & $2 F$ & 45 & 51 & 97 \\
\hline$B$ & $8 \mathrm{C}$ & $7 A$ & $A C$ & 75 & $2 A$ & $\mathrm{BD}$ & $A D$ & E3 & BE & CF & 47 & 37 & E1 & 93 & C4 & C9 \\
\hline C & CE & C7 & C6 & $\mathrm{OC}$ & E4 & 04 & 76 & 69 & 43 & $\mathrm{DE}$ & A7 & EF & OD & 84 & 92 & 22 \\
\hline D & 50 & 68 & 57 & 1B & $5 \mathrm{D}$ & FA & EB & 36 & $8 \mathrm{E}$ & $8 \mathrm{D}$ & E9 & $7 F$ & 4B & 71 & F6 & 03 \\
\hline E & 9A & D5 & 60 & $1 \mathrm{C}$ & F5 & $1 \mathrm{E}$ & $\mathrm{CD}$ & A4 & $5 \mathrm{~A}$ & $8 B$ & 73 & 16 & 46 & 64 & $9 \mathrm{E}$ & 00 \\
\hline & 960 & DF & 08 & 70 & 94 & 30 & 59 & 53 & 50 & $\mathrm{CC}$ & 67 & 40 & $9 D$ & 35 & $3 \mathrm{E}$ & $3 F$ \\
\hline
\end{tabular}

\begin{tabular}{|c|c|c|c|c|c|c|c|c|c|c|c|c|c|c|c|c|}
\hline & 0 & 1 & 2 & 3 & 4 & 5 & 6 & 7 & 8 & 9 & A & B & $\mathrm{C}$ & D & $\mathrm{E}$ & $F$ \\
\hline 0 & EF & 3D & 53 & DF & C5 & 68 & 22 & 57 & F2 & A5 & $7 E$ & $3 \mathrm{E}$ & C3 & $\mathrm{CC}$ & A4 & F1 \\
\hline 1 & 38 & 1C & 08 & $O E$ & $2 \mathrm{E}$ & OF & EB & 7B & 7A & 17 & 20 & D3 & E3 & 7C & E5 & $6 \mathrm{~A}$ \\
\hline 2 & 06 & $4 C$ & CF & 64 & 95 & $3 \mathrm{~F}$ & A0 & $1 \mathrm{~F}$ & 61 & 76 & B4 & 5D & $6 C$ & 52 & OA & $A C$ \\
\hline 3 & F5 & 47 & 90 & $6 \mathrm{E}$ & 45 & FD & D7 & BB & 60 & 39 & 88 & 92 & 94 & 11 & $\mathrm{FE}$ & $\mathrm{FF}$ \\
\hline 4 & FB & 30 & 98 & C8 & $9 \mathrm{~B}$ & $A D$ & EC & BA & $2 C$ & $4 \mathrm{~A}$ & $8 \mathrm{E}$ & DC & $2 A$ & $9 \mathrm{C}$ & 72 & $8 D$ \\
\hline 5 & DO & $\mathrm{AE}$ & 80 & F7 & 31 & 4B & 51 & D2 & $1 \mathrm{E}$ & F6 & E8 & AA & F8 & D4 & $1 B$ & 28 \\
\hline 6 & E2 & 93 & $8 \mathrm{~F}$ & 00 & ED & $5 A$ & 27 & FA & D1 & C7 & 13 & 7D & 1D & 05 & $1 \mathrm{~A}$ & 56 \\
\hline 7 & F3 & DD & $6 \mathrm{~B}$ & EA & 91 & B3 & C6 & 32 & 96 & OD & B1 & $2 B$ & 01 & 50 & 65 & DB \\
\hline 8 & 67 & 04 & $8 \mathrm{~A}$ & 07 & $C D$ & 74 & 81 & 55 & 78 & A1 & 83 & E9 & BO & D9 & D8 & 14 \\
\hline 9 & 70 & 10 & CE & BD & $\mathrm{F} 4$ & 40 & Fo & AF & 77 & A6 & EO & 35 & 4D & FC & EE & 69 \\
\hline A & 21 & 16 & $8 \mathrm{~B}$ & 25 & E7 & A3 & 02 & CA & A8 & 15 & 73 & $4 \mathrm{E}$ & B2 & B6 & $3 \mathrm{~A}$ & 34 \\
\hline B & 09 & A9 & 24 & 18 & $3 C$ & 79 & 84 & 86 & $5 F$ & 99 & 46 & 82 & 58 & B5 & B8 & 66 \\
\hline$c$ & 12 & 48 & $O C$ & 37 & $\mathrm{BE}$ & $8 \mathrm{C}$ & C2 & C1 & 42 & BF & 43 & 89 & F9 & E6 & $\mathrm{CO}$ & B9 \\
\hline D & 23 & 44 & 41 & 49 & $2 F$ & E1 & $9 \mathrm{~A}$ & 75 & $5 C$ & $7 F$ & 33 & AB & $6 \mathrm{~F}$ & 63 & C9 & 19 \\
\hline $\mathrm{E}$ & 97 & BC & $6 \mathrm{D}$ & B7 & $\mathrm{C4}$ & 03 & OB & 26 & 87 & DA & $4 F$ & D6 & A7 & $9 \mathrm{E}$ & 85 & CB \\
\hline & $9 \mathrm{~F}$ & 71 & $5 \mathrm{E}$ & 90 & 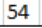 & E4 & D & 3B & 62 & A2 & D5 & $2 \mathrm{D}$ & 59 & 36 & $5 B$ & 29 \\
\hline
\end{tabular}

Table 4.14: S-box and Inverse S-box for $x^{8}+x^{7}+x^{6}+x+1$

\begin{tabular}{|llllllllllll|l|l|l|l|l|l|l|l|}
0 & 1 & 2 & 3 & 4 & 5 & 6 & 7 & 8 & 9 & A & B & C & D & E & $F$ \\
\hline
\end{tabular}

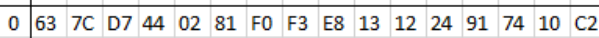

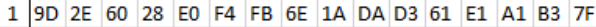
\begin{tabular}{lllllllllll|l|l|l|l|l|l|l}
2 & 27 & 45 & $\mathrm{FE}$ & 09 & $\mathrm{E} 2$ & $\mathrm{C} 3$ & $\mathrm{C} 6$ & OF & 99 & $\mathrm{CE}$ & A8 & 26 & 14 & BO & DE & OA
\end{tabular} $\begin{array}{llllllllllllllllll}3 & \text { E4 } & \text { CF } & \text { BF } & 58 & 3 B & \text { A5 } & 62 & 1 C & 19 & \text { B5 } & 39 & 46 & 30 & 90 & 56 & 3 \mathrm{C}\end{array}$ \begin{tabular}{llllllll|l|l|l|l|l|l|l|l|l}
4 & $7 A$ & A9 & 70 & 35 & AD & $7 B$ & $6 D$ & 32 & 98 & 41 & 33 & 03 & $8 A$ & 52 & 55 & C9
\end{tabular} \begin{tabular}{l|llllllllllllllllll}
5 & $1 \mathrm{E}$ & D6 & $8 \mathrm{E}$ & F8 & BD & A7 & FA & 88 & D8 & 64 & B1 & $6 \mathrm{C}$ & 86 & 67 & EC & 21
\end{tabular}

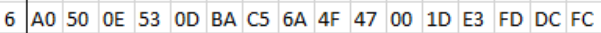

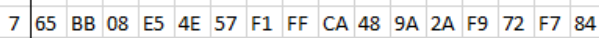
8 EF $3 E \begin{array}{lllllllllllllll} & 3 D & 07 & \text { EA } & 2 F & 73 & 93 & 04 & \text { AF } & 6 F & 85 & 5 F & 76 & \text { CB } & 23\end{array}$

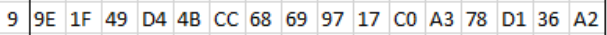
A \begin{tabular}{llllll|lllllllllll} 
& DD & 82 & $8 D$ & AE & $8 C$ & 95 & $3 F$ & $0 C$ & $9 B$ & 01 & $4 A$ & 94 & $8 B$ & 96 & 06
\end{tabular}

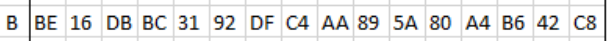
$\begin{array}{lllllllllllllllllll}\text { C } & \text { B9 } & \text { F6 } & \text { C1 } & 25 & \text { D5 } & 51 & 40 & 77 & 54 & 7 E & B 4 & 9 C & 0 B & 1 B & \text { E7 } & 6 B\end{array}$

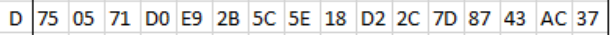

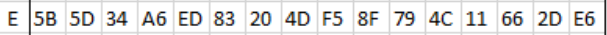
\begin{tabular}{l|llllllllllllllllll}
$\mathrm{F}$ & $\mathrm{B} 7$ & 59 & $\mathrm{CD}$ & 22 & $9 \mathrm{~F}$ & 38 & $\mathrm{C} 7$ & $\mathrm{~B} 2$ & 15 & $3 \mathrm{~A}$ & $\mathrm{~EB}$ & $\mathrm{EE}$ & 29 & $\mathrm{~B} 8$ & $\mathrm{AB}$ & $\mathrm{F} 2$ \\
\hline
\end{tabular} \begin{tabular}{|l|l|l|l|l|l|l|l|l|l|l|l|l|l|l|l|l|} 
& 0 & 1 & 2 & 3 & 4 & 5 & 6 & 7 & 8 & 9 & A & B & C & D & E & $F$ \\
\hline
\end{tabular}

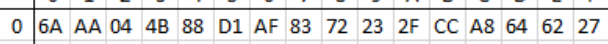

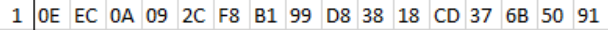

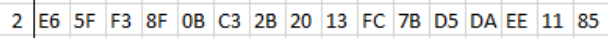
\begin{tabular}{l|lllllllllllllllllll}
3 & $3 \mathrm{C}$ & B4 & 47 & $4 \mathrm{~A}$ & $\mathrm{E} 2$ & 43 & $9 \mathrm{E}$ & $\mathrm{DF}$ & $\mathrm{F} 5$ & $3 \mathrm{~A}$ & $\mathrm{~F} 9$ & 34 & $3 \mathrm{~F}$ & 82 & 81 & A7
\end{tabular}

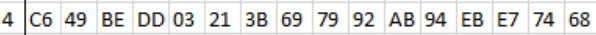
$\begin{array}{llllllllllllllllll}5 & 61 & \text { C5 } & 4 D & 63 & \text { C8 } & 4 E & 3 E & 75 & 33 & \text { F1 } & \text { BA E } & \text { E0 } & \text { D6 } & \text { E1 } & \text { D7 } & 8 C\end{array}$

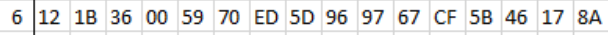

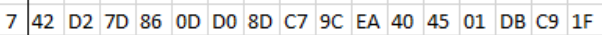
8 BB 05 A2 E5 $7 F$ 8B 5 5C DC 57 B9 4 4C AD A5 A3 52 E9

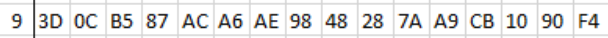
$\begin{array}{lllllllllllllllll}\text { A } & 60 & 1 D & 9 F & 9 B & B C & 35 & \text { E3 } & 55 & \text { 2A } & 41 & \text { B8 } & \text { FE } & \text { DE } 44 & \text { A4 } & 89\end{array}$ \begin{tabular}{l|llllllllllllllllllllll} 
B & 2D & $5 A$ & F7 & 1E & CA & 39 & BD & F0 & FD & C0 & 65 & 71 & B3 & 54 & BO & 32
\end{tabular} $\begin{array}{lllllllllllllllllllll}\text { C } & 9 A & \text { C2 } & \text { OF } & 25 & \text { B7 } & 66 & 26 & \text { F6 } & \text { BF } & 4 F & 78 & 8 E & 95 & \text { F2 } & 29 & 31\end{array}$

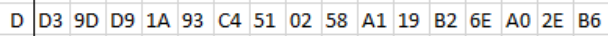

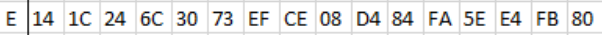

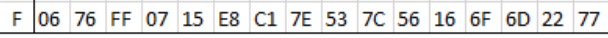


Table 4.15: S-box and Inverse S-box for $x^{8}+x^{7}+x^{6}+x^{3}+x^{2}+x+1$

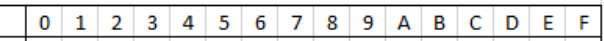
\begin{tabular}{l|llllllllllllllllll|}
0 & 63 & $7 \mathrm{C}$ & 95 & 38 & 61 & $\mathrm{FB}$ & $\mathrm{CE}$ & $9 \mathrm{~A}$ & 62 & $\mathrm{CB}$ & 56 & 83 & $\mathrm{CC}$ & $2 \mathrm{~A}$ & $9 \mathrm{~F}$ & 45
\end{tabular} $\begin{array}{llllllllllllllllllll}1 & \text { E3 } & 2 \mathrm{~F} & 37 & 6 \mathrm{~F} & \mathrm{~F} 9 & 41 & 13 & \text { 0A } & \mathrm{CD} & \mathrm{FF} & \mathrm{C} 7 & \mathrm{AA} & 64 & 6 \mathrm{D} & 70 & \mathrm{AC}\end{array}$

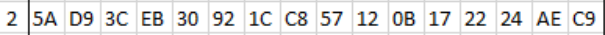

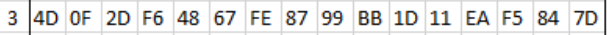
446696 3E $D 1$ B5 54 5E EE CA 4C 9B D4 DC B2 B6 $1 \mathrm{~A}$ \begin{tabular}{lllllllllllll|l|l|l|l|l}
5 & 79 & 44 & A2 & 47 & $2 E$ & EF & 59 & 14 & C3 & $3 F$ & B9 & B8 & 85 & 21 & 36 & 31
\end{tabular}

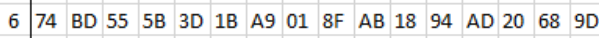

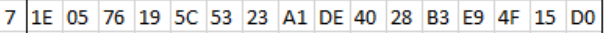

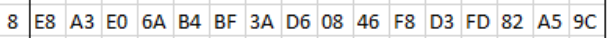
$9 \begin{array}{llllllllllllllllllll}9 & \mathrm{~B} 7 & 0 \mathrm{E} & \mathrm{F} 4 & \mathrm{BE} & 1 \mathrm{~F} & \mathrm{E} 4 & \mathrm{C} 1 & 51 & \mathrm{C} 5 & 73 & \mathrm{~F} 2 & 39 & 89 & \mathrm{~A} 4 & \mathrm{~A} 6 & 8 \mathrm{C}\end{array}$ \begin{tabular}{l|l|l|l|l|l|l|l|l|l|l|l|l|l|l|l|l} 
A & $6 E$ & 52 & F0 & 29 & FA & DA 71 & 10 & BC EC & 25 & B1 & 07 & $9 E$ & D 8 & $4 B$
\end{tabular}

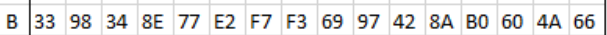
\begin{tabular}{llllllllll|l|l|l|l|l|l|l|l|l} 
C & 91 & CO & $0 C$ & CF & 78 & $0 D$ & 06 & 88 & 35 & E7 & 26 & DB & $7 F$ & $7 A$ & $2 B$ & DF
\end{tabular} $\begin{array}{lllllllllllllllllll}D & 6 C & \text { E5 } & 7 E & 00 & \text { A7 } & 93 & \text { E1 } & 2 C & 04 & 3 B & \text { C2 } & \text { D7 } & \text { E6 } & \text { F1 } & 65 & \text { AF }\end{array}$

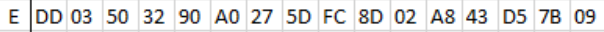
\begin{tabular}{l|lllllllllllllllllll} 
& F & $C 4$ & $E D$ & $8 B$ & 81 & $C 6$ & 80 & 72 & 16 & $5 F$ & $4 E$ & 75 & D2 & 58 & $6 B$ & $B A$ & 49 \\
\hline
\end{tabular}

\begin{tabular}{|c|c|c|c|c|c|c|c|c|c|c|c|c|c|c|c|c|}
\hline & 0 & 1 & 2 & 3 & 4 & 5 & 6 & 7 & 8 & 9 & A & B & C & D & $E$ & \\
\hline 0 & D3 & 67 & EA & E1 & D8 & 71 & C6 & $A C$ & 88 & EF & 17 & $2 \mathrm{~A}$ & C2 & C5 & 91 & 31 \\
\hline 1 & A7 & 3B & 29 & 16 & 57 & $7 E$ & F7 & $2 B$ & $6 \mathrm{~A}$ & 73 & $4 \mathrm{~F}$ & 65 & 26 & $3 A$ & 70 & 94 \\
\hline 2 & $6 \mathrm{D}$ & $5 D$ & $2 \mathrm{C}$ & 76 & $2 \mathrm{D}$ & AA & CA & E6 & $7 \mathrm{~A}$ & A3 & OD & CE & D7 & 32 & 54 & 11 \\
\hline 3 & 24 & $5 F$ & E3 & BO & B2 & $\mathrm{C} 8$ & $5 \mathrm{E}$ & 12 & 03 & $9 \mathrm{~B}$ & 86 & D9 & 22 & 64 & 42 & 59 \\
\hline 4 & 79 & 15 & BA & EC & 51 & OF & 89 & 53 & 34 & FF & $\mathrm{BE}$ & AF & 49 & 30 & F9 & 7D \\
\hline 5 & E2 & 97 & A1 & 75 & 45 & 62 & $O A$ & 28 & FC & 56 & 20 & 63 & 74 & E7 & 46 & F8 \\
\hline 6 & $B D$ & 04 & 08 & 00 & $1 \mathrm{C}$ & $\mathrm{DE}$ & BF & 35 & $6 \mathrm{E}$ & B8 & 83 & FD & DO & 1D & A0 & 13 \\
\hline 7 & $1 \mathrm{E}$ & A6 & F6 & 99 & 60 & FA & 72 & B4 & $\mathrm{C} 4$ & 50 & $C D$ & $\mathrm{EE}$ & 01 & $3 \mathrm{~F}$ & D2 & CC \\
\hline 8 & F5 & F3 & $8 \mathrm{D}$ & $\mathrm{OB}$ & $3 \mathrm{E}$ & $5 C$ & 40 & 37 & C7 & 9C & BB & F2 & $9 \mathrm{~F}$ & E9 & B3 & 68 \\
\hline 9 & E4 & $\mathrm{CO}$ & 25 & D5 & $6 \mathrm{~B}$ & 02 & 41 & B9 & B1 & 38 & 07 & $4 \mathrm{~A}$ & $8 \mathrm{~F}$ & $6 \mathrm{~F}$ & $A D$ & $O E$ \\
\hline A & E5 & 77 & 52 & 81 & $9 D$ & $8 \mathrm{E}$ & $9 \mathrm{E}$ & D4 & EB & 66 & $1 \mathrm{~B}$ & 69 & $1 F$ & $6 \mathrm{C}$ & $2 \mathrm{E}$ & DF \\
\hline B & $B C$ & $\mathrm{AB}$ & $4 D$ & 7B & 84 & 44 & $4 \mathrm{E}$ & 90 & $5 B$ & $5 A$ & $\mathrm{FE}$ & 39 & A8 & 61 & 93 & 85 \\
\hline C & C1 & 96 & DA & 58 & F0 & 98 & F4 & $1 \mathrm{~A}$ & 27 & $2 F$ & 48 & 09 & OC & 18 & 06 & C3 \\
\hline D & $7 F$ & 43 & FB & $8 B$ & 4B & ED & 87 & DB & $\mathrm{AE}$ & 21 & A5 & CB & $4 C$ & EO & 78 & CF \\
\hline$E$ & 82 & D6 & B5 & 10 & 95 & D1 & DC & $\mathrm{Cg}$ & 80 & 7C & $3 \mathrm{C}$ & 23 & A9 & F1 & 47 & 55 \\
\hline & A2 & DD & $9 \mathrm{~A}$ & B/ & 92 & 3D & 33 & B6 & $8 \mathrm{~A}$ & 14 & A4 & 05 & E8 & $8 \mathrm{C}$ & 36 & 19 \\
\hline
\end{tabular}

Table 4.16: S-box and Inverse S-box for $x^{8}+x^{7}+x^{6}+x^{5}+x^{2}+x+1$ \begin{tabular}{|cccccccccccccccccc}
0 & 1 & 2 & 3 & 4 & 5 & 6 & 7 & 8 & 9 & A & B & C & D & E & F \\
\hline & 63 & $7 C$ & 18 & 31 & $2 A$ & $0 A$ & $4 A$ & $F A$ & $C 7$ & $E B$ & 23 & $A D$ & 03 & $3 A$ & $5 B$ & $B B$
\end{tabular}

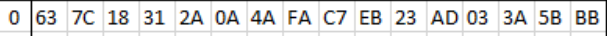

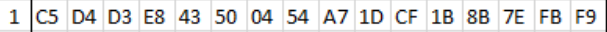

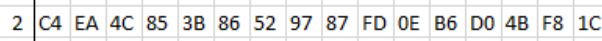
$\begin{array}{llllllllllllllllll}3 & 01 & \text { F2 } & 5 \mathrm{C} & \mathrm{F} 1 & \mathrm{Cl} & 1 \mathrm{~A} & \mathrm{AB} & 6 \mathrm{~B} & 17 & \mathrm{D} 6 & 19 & 14 & \mathrm{DB} & 9 \mathrm{~F} & \mathrm{DA} & 8 \mathrm{D}\end{array}$ $4 \begin{array}{llllllllllllllll}44 & \text { C6 } & 53 & \text { A1 } & \text { F4 } & 9 B & \text { E4 } & 37 & 4 F & \text { EE } & 65 & 57 & \text { OF } & 4 E & \text { ED } & 71\end{array}$ $\begin{array}{lllllllllllllllll}5 & \text { E5 } & 21 & 2 C & \text { B4 } & \text { D5 } & \text { B5 } & 89 & \text { C9 } & \text { BA } & 3 C & 83 & 69 & 5 A & \text { CD DC } & \text { EC }\end{array}$ \begin{tabular}{llllllllllll|l|l|l|l|l}
6 & A6 & 26 & $5 F$ & BC & FC & 99 & DE & 12 & 32 & 68 & 2B & 60 & F3 & EF & 67 & 98
\end{tabular}

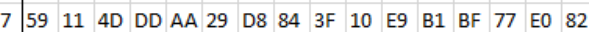

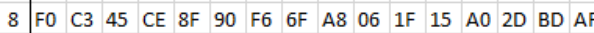
\begin{tabular}{l|l|l|l|l|l|l|l|l|l|l|l|l|l|l|l|l}
9 & 75 & B8 & A5 & B2 & 94 & 09 & 79 & A3 & 55 & $3 E$ & F5 & 80 & 24 & E3 & $6 A$ & 02
\end{tabular} \begin{tabular}{l|lllllllllllllllllll} 
A & 20 & 51 & 42 & 07 & 30 & $8 \mathrm{E}$ & 88 & $\mathrm{C} 2$ & $\mathrm{CC}$ & $\mathrm{B} 3$ & 08 & 96 & 16 & 61 & 36 & $\mathrm{CA}$
\end{tabular}

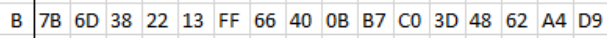
$\begin{array}{llllllllllllllllll}C & 81 & 7 F & 35 & 00 & 7 D & 7 A & 8 C & 9 C & A C & F 7 & 1 E & 6 E & 49 & \text { A2 } & 2 F & 6 C\end{array}$

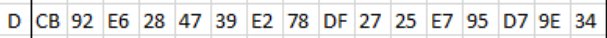
$\begin{array}{lllllllllllllllllll}\text { E } & 8 A & 41 & \text { AE } & 70 & 74 & 33 & \text { C8 } & 5 E & 73 & 91 & 46 & \text { A9 } & \text { BE } & 9 A & 64 & \text { E1 }\end{array}$ \begin{tabular}{llllllllllllllllllll} 
F & B9 & 58 & $2 E$ & $5 D$ & $D 2$ & $D 1$ & $F E$ & 72 & OD & 05 & $9 D$ & $0 C$ & 56 & B0 & 93 & 76 \\
\hline
\end{tabular} \begin{tabular}{|l|l|l|l|l|l|l|l|l|l|l|l|l|l|l|l|l|}
\hline & 0 & 1 & 2 & 3 & 4 & 5 & 6 & 7 & 8 & 9 & A & B & C & D & E & F \\
\hline
\end{tabular}

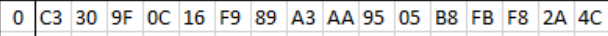

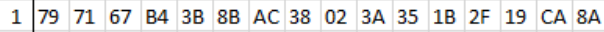
2 A0 51 B3 0 OA 9 9C DA 61 D9 D3 75 04 6 AA 52 8D F2 $\begin{array}{lllllllllllllllll}3 & \text { A4 } & 03 & 68 & \text { E5 } & \text { DF } & \text { C2 } & \text { AE } & 47 & \text { B2 } & \text { D5 } & \text { OD } & 24 & 59 & \text { BB } & 99 & 78\end{array}$

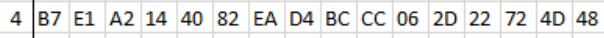
$\begin{array}{lllllllllllllllllllll}5 & 15 & \text { A1 } & 26 & 42 & 17 & 98 & \text { FC } & 4 B & \text { F1 } & 70 & 5 C & \text { 0E } & 32 & \text { F3 } & \text { E7 } & 62\end{array}$

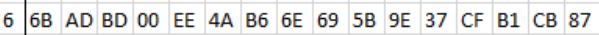
\begin{tabular}{l|l|l|l|l|l|l|l|l|l|l|l|l|l|l|l|l}
7 & E3 & $4 F$ & F7 & E8 & E4 & 90 & FF & $7 D$ & D7 & 96 & C5 & B0 & 01 & C4 & $1 D$ & C1
\end{tabular}

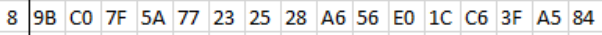

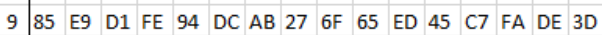
\begin{tabular}{l|llllllllllllllllll} 
A & $8 \mathrm{C}$ & 43 & $\mathrm{CD}$ & 97 & $\mathrm{BE}$ & 92 & 60 & 18 & 88 & $\mathrm{~EB}$ & 74 & 36 & $\mathrm{C} 8$ & 0B & E2 & $8 \mathrm{~F}$
\end{tabular}

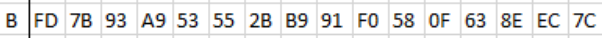
$\begin{array}{llllllllllllllllll}C & \text { BA } & 34 & \text { A7 } & 81 & 20 & 10 & 41 & 08 & \text { E6 } & 57 & \text { AF } & \text { D0 } & \text { A8 } & 5 D & 83 & \text { AA }\end{array}$

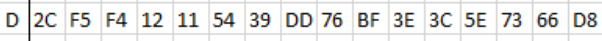

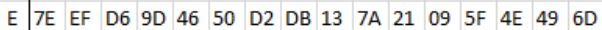
\begin{tabular}{lllllllllllllllllll}
$\mathrm{F}$ & 80 & 33 & 31 & $6 \mathrm{C}$ & 44 & $9 \mathrm{~A}$ & 86 & $\mathrm{C} 9$ & $2 \mathrm{E}$ & $1 \mathrm{~F}$ & 07 & $1 \mathrm{E}$ & 64 & 29 & $\mathrm{~F} 6$ & $\mathrm{~B} 5$ \\
\hline
\end{tabular}

Table 4.17: S-box and Inverse S-box for $x^{8}+x^{7}+x^{6}+x^{5}+x^{4}+x^{2}+1$

\begin{tabular}{l|llllllllllllllllllllll} 
& 0 & 1 & 2 & 3 & 4 & 5 & 6 & 7 & 8 & 9 & A & B & C & D & E & $F$ \\
\hline
\end{tabular} \begin{tabular}{l|lllllllllllllllllll}
\hline 0 & 63 & $7 \mathrm{C}$ & $\mathrm{FF}$ & $8 \mathrm{~B}$ & $2 \mathrm{D}$ & $3 \mathrm{~B}$ & 17 & $7 \mathrm{E}$ & 57 & 97 & $4 \mathrm{~F}$ & $\mathrm{C} 8$ & 59 & $\mathrm{~F} 4$ & $\mathrm{FE}$ & 49
\end{tabular} $\begin{array}{llllllllllllllllllll}1 & 79 & 98 & \text { OA } & 45 & 75 & 72 & \text { B6 } & \text { F3 } & 6 D & \text { EE } & \text { A8 } & \text { D2 } 2 & \text { AD } & 18 & 65 & 58\end{array}$

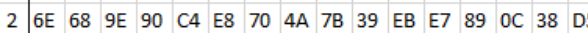

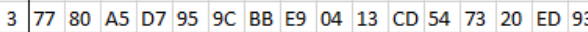
4 F6 33 E6 5F 8 8E AF 9A 40 A3 A7 B5 AE EA 6C E4 23 5 6 6 F DD 4E $\begin{array}{llllllllllllllllllll}6 & 7 \mathrm{~A} & 37 & 92 & 87 & 00 & 32 & 2 \mathrm{~A} & \mathrm{E} 2 & 0 \mathrm{~B} & 99 & 8 \mathrm{~F} & \mathrm{BE} & 1 \mathrm{C} & 22 & 35 & 1 \mathrm{~A}\end{array}$

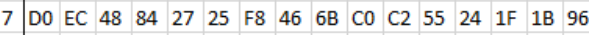
8 A9 CF 4B B7 A1 81 7D AA 86 BF 05 FD $9 F$ F1 71 E1 C6 \begin{tabular}{l|l|l|l|l|l|l|l|l|l|l|l|l|l|l|l|l|l}
9 & 10 & 69 & 01 & FB & 08 & $3 \mathrm{E}$ & 85 & F9 & B4 & $9 D$ & F7 & FC & A0 & 14 & 43 & BC
\end{tabular} $\begin{array}{llllllllllllllllll}A & 76 & 1 D & 2 F & 4 D & F 5 & 3 F & 2 E & 30 & \text { DB } & \text { E3 } & \text { B9 } & 19 & 42 & \text { E0 } & 62 & \text { B1 }\end{array}$ B D9 $5651 \quad 74$ AB FA B8 5C A6 D6 3A CA 4728 DA 5E

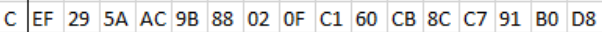

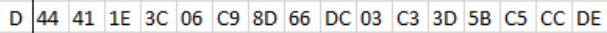

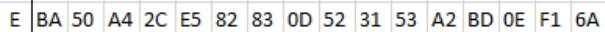
$\begin{array}{lllllllllllllllllll}\mathrm{F} & 67 & 64 & \mathrm{~B} 2 & 09 & \mathrm{~B} 3 & 15 & 78 & 26 & \mathrm{D} 3 & 7 \mathrm{~F} & 5 \mathrm{D} & 94 & 4 \mathrm{C} & 11 & 8 \mathrm{~A} & 12\end{array}$ \begin{tabular}{lllllllllllllllllllll}
0 & 1 & 2 & 3 & 4 & 5 & 6 & 7 & 8 & 9 & A & B & C & D & E & F \\
\hline
\end{tabular}

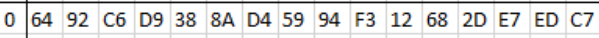
$\begin{array}{llllllllllllllllll}1 & 90 & \text { FD } & \text { FF } & 39 & 9 D & \text { F5 } & 58 & 06 & \text { 1D } & \text { AB } & 6 F & 7 E & 6 C & \text { A1 } & \text { D2 } & 7 D\end{array}$ $\begin{array}{lllllllllllllllllll}2 & 3 \mathrm{D} & 56 & 6 \mathrm{D} & 4 \mathrm{~F} & 7 \mathrm{C} & 75 & \mathrm{~F} 7 & 74 & \mathrm{BD} & \mathrm{C} 1 & 66 & 5 \mathrm{E} & \mathrm{E} 3 & 04 & \text { A6 } & \text { A2 }\end{array}$ \begin{tabular}{l|l|l|l|l|l|l|l|l|l|l|l|l|l|l|l|l}
3 & A7 & E9 & 65 & 41 & 54 & $6 E$ & $5 F$ & 61 & $2 E$ & 29 & BA & 05 & D3 & DB & 95 & A5
\end{tabular} \begin{tabular}{l|l|l|l|l|l|l|l|l|l|l|l|l|l|l|l|l|l}
4 & 47 & D1 & AC & $9 E$ & D0 & 13 & 77 & BC & 72 & OF & 27 & 82 & FC & A3 & 52 & OA
\end{tabular}

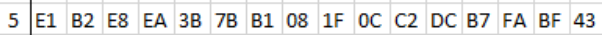
$\begin{array}{llllllllllllllllll}6 & \text { C9 } & 57 & \text { AE } & 00 & \text { F1 } & 1 E & \text { D7 } & \text { F0 } & 21 & 91 & \text { EF } & 78 & \text { 4D } & 18 & 20 & 50\end{array}$ \begin{tabular}{lllll|l|l|l|l|l|l|l|l|l|l|l|l|}
7 & 26 & $8 \mathrm{D}$ & 15 & $3 \mathrm{C}$ & $\mathrm{B} 3$ & 14 & A0 & 30 & $\mathrm{~F} 6$ & 10 & 60 & 28 & 01 & 86 & 07 & $\mathrm{~F} 9$
\end{tabular} \begin{tabular}{lllllllllll|llllllll}
8 & 31 & 85 & E5 & E6 & 73 & 96 & 88 & 63 & C5 & $2 \mathrm{C}$ & $\mathrm{FE}$ & 03 & $\mathrm{CB}$ & $\mathrm{D} 6$ & 44 & $6 \mathrm{~A}$
\end{tabular}

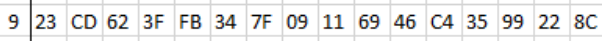

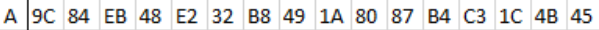

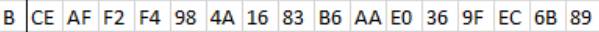
C 79 C8 $7 A$ DA 24 DD 8F CC OB D5 BB CA DE 3 AA 5 5C 81

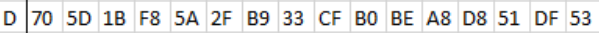

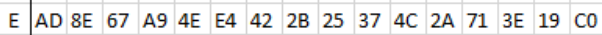
$\begin{array}{lllllllllllllllllll}\mathrm{F} & 55 & \mathrm{EE} & 5 \mathrm{~B} & 17 & \mathrm{OD} & \mathrm{A} 4 & 40 & 9 \mathrm{~A} & 76 & 97 & \mathrm{~B} 5 & 93 & 9 \mathrm{~B} & 8 \mathrm{~B} & \mathrm{OE} & 02\end{array}$ 
selection.

- Mixcolumn layer uses the selected polynomial for modulo reduction.

\subsection{Methodology}

The customized approach is as follows: The coefficients of every equation from the table, are fed as inputs to a gate block. For instance, 9-bit data representation of the polynomial $x^{8}+x^{5}+x^{3}+x+1$ is 100101011 . In this algorithm, the gate layer uses an XOR function. In future developments, this could be used as a reference to build the layer with sophisticated logic gate functions. An XOR operation performed between the input bit and another 9-bit input in the gate function results in a 9-bit output as shown in Fig. 4-1. The function acts as an added layer of security to increase the complexity.

Using an RNG (Random Number Generator) function, a 512-bit number is generated. Every 9-bit from the random number is compared to the output of the gate function, one after the other till a match is found. The scan happens in a clockwise direction. Since 512-bits could be split into 56 nine bit numbers only, the remaining 8-bits and the first bit of the random number form a 9-bit pair. The corresponding primitive polynomial of the matched output bit is the polynomial used for the encryption algorithm. If no match is found, the first equation in the table is chosen as the primitive polynomial and as for the key, the 128 most significant bits of the 512-bit number is selected by default.

The 128-bit key is selected from the bits of the 512-bit random number, starting

with the bit after the block of 9-bits that selected the primitive polynomial. The 128-bits are selected cyclically, i.e., if we reach the last bit when we scan the 512-bit random number, then we continue into the first bit and beyond until we select all the 128 bits needed for the key. Fig. 5-2 is an example for key establishment protocol. 


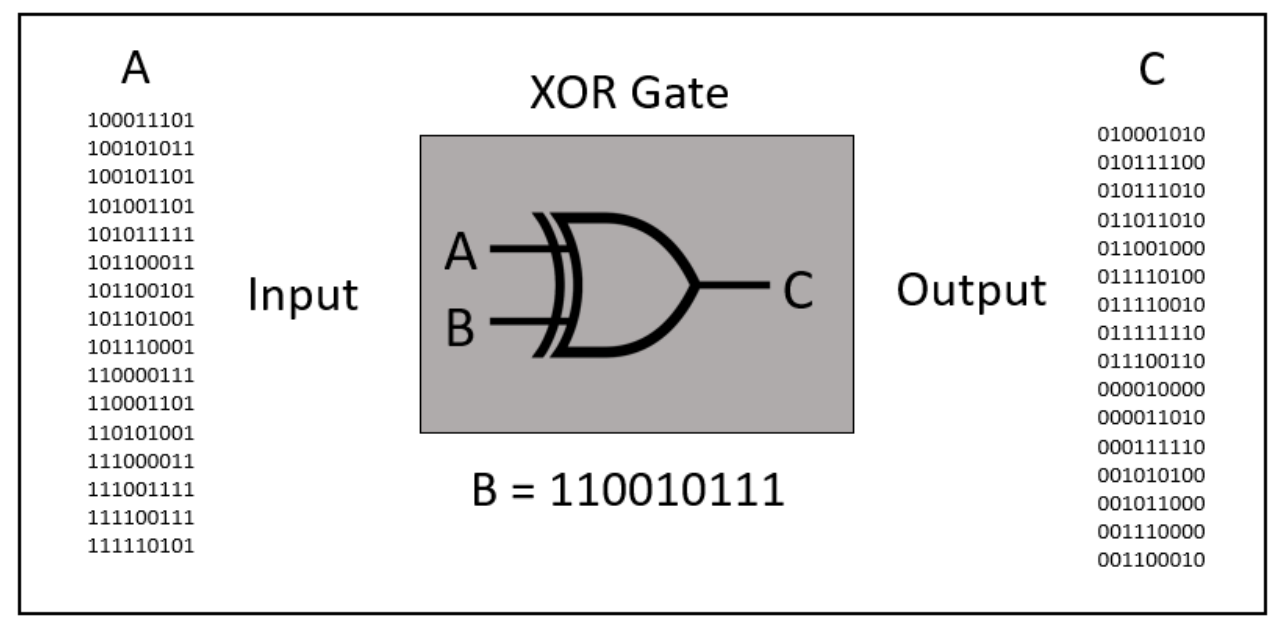

Figure 4-1: Gate Function Block

Once the primitive polynomial and 128-bit key are determined, the proposed algorithm differs from AES in the following ways:

- The Byte-substitution transformation retrieves one among the sixteen S-boxes and sixteen inverse S-boxes from the stored tables, with the selected polynomial as a base. Whereas in AES only one S-box and inverse S-box of the standard polynomial is used.

- In the mixcolumn transformation, the selected polynomial is used by the matrix and inverse matrix operations, unlike AES that uses only one polynomial.

- In AES, the 128-bit key is randomly generated using an RNG. In the proposed algorithm the 128-bit key is generated using the described key selection process.

Both the encryption and decryption algorithms are set up in all the devices and are enabled depending on whether the device is a sender or receiver. The Shiftrow layer operations remain the same in both the algorithms. 


\section{Random Key (512-bit)}

100110011011001101000011010101001101111111100001011100111

111000001100011110010000100100000001100010011011110001001 010010110111100110101001110100010110100000010100110111101 001010001100111011101111100011100110111010101010000100110 101110000010111101111010000010010011111111011101101000000 110101111001111110111011100000011100011010111100011101101 010110001100100110101000101000101100100010101111110011110 001011111001011101100100110100000101001101010111010110011 00100011111010101101101000101011101010100001100001110001

\section{The 128-bit key to Algorithm}

111111011101110000001110001101011110001110110101011000110 010011010100010100010110010001010111111001111000101111100 10111011001001

Figure 4-2: Protocol for Key Establishment

\subsection{Communication Protocol}

Once the algorithms are put together in the communicating devices, the protocol on the sender's end works as shown in Fig. 4-3:

1. Device A computes its private (a) and public keys $\left(K_{A}\right)$.

2. It composes the message $(\mathrm{X})$. The message digest $(\mathrm{Z})$ of the message is produced using SHA-256 hash function $(\mathrm{h}(\mathrm{x}))$.

3. The hash is encrypted (U) with the private key of the device A.

4. The 512-bits (R) are produced using a random number generator and are encrypted using the public key shared by device B $\left(K_{B}\right)$. 
Device A

- Generates pair of keys a, $K_{A}$

- Composes the message $X$

- Computes a hash using SHA-256

$$
\stackrel{\mathrm{X}}{\longrightarrow} \mathrm{h}(\mathbf{X}) \stackrel{\mathrm{Z}}{\longrightarrow}
$$

- Encrypts Z with private key

$$
\mathbf{U}=\mathbf{Z}^{\mathbf{a}}
$$

- Generates 512-bit random number $\mathbf{R}$

- Encrypts session key with B's public key as $\mathrm{Y}_{\mathrm{B}}=R^{K_{B}}$

- Extracts 128-bit key using the key selection process

- Encrypts X using the customized algorithm, $\mathbf{Y}=\mathbf{A E S}(\mathbf{X})$

Device B

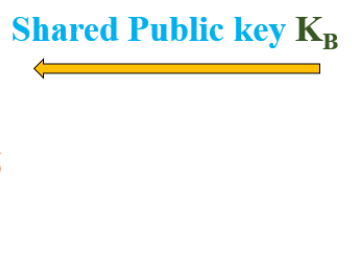

$\stackrel{\text { Shared Public key } \mathbf{K}_{\mathrm{A}}}{\longrightarrow}$

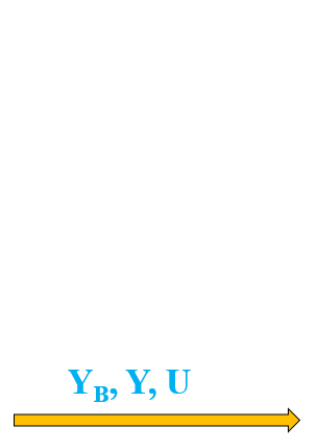

- Generates pair of keys $b, K_{B}$

- Decrypts $\mathrm{R}$ using private key $\mathbf{R}=\mathbf{Y}_{\mathbf{B}} \mathbf{b}$

- Extracts the 128-bit key using the same selection process

- Decrypts the message $\mathbf{X}=\operatorname{AES}^{-1}(\mathrm{Y})$

- Decrypts the hash using A's public key, $Z=U^{K_{A}}$

- Verifies authenticity of message

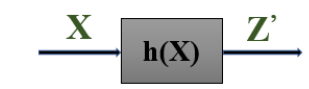

If $\mathrm{Z}^{\prime}=\mathrm{Z}^{\prime}$ is true or false

Figure 4-3: Communication protocol between devices

5. The primitve polynomial and the 128-bit key are extracted using the customized algorithm.

6. The message is encrypted using the selected key (128-bit). Finally, the encrypted message $(\mathrm{Y})$, a random number $\left(Y_{B}\right)$ and hash $(\mathrm{U})$ are sent to device B.

The decryption at the receiver's end are as follows:

1. Device B decrypts the random number using its private key (b).

2. Using the algorithm, it deduces the 128-bit key and decrypts the message.

3. Decrypts the hash using the shared public key of device $\mathrm{A}\left(K_{A}\right)$

4. Creates message digest using the same hash function. 
5. Compares the decrypted and computed hashes to check the integrity of the message.

From this protocol, we understand that the communicating devices need to possess public keys of the devices they want to connect. They need to be sure about sharing the public key with the intended users only, as there are chances of spoofing identities. The complex feature of the algorithm ensures safe transmission as attackers cannot decrypt the text despite having access to keys. However, our intention is to increase security by authenticating the keys.

\subsection{Authentication Protocol}

Let us assume a scenario wherein two users want to communicate and are ready to share their public keys. A Man In The Middle interrupts the communication and shares the public key as the intended user. He locks the hash of the message with his private key. When the user verifies the hash of the message, it results to be right as the MITM's private key matches to the public shared by him. To overcome this situation, we introduce a centralized server to distribute the keys. All the devices participating in the network are assigned unique identities [30]. A centralized server maintains a record of the unique ID numbers and public keys of all the devices known by their user names $R_{A}, R_{B}, R_{C}$ and so on.

The server possesses a public $\left(K_{S}\right)$ and private key $(\mathrm{s})$ as well. The public key of the server is pre-stored into all the devices participating in the network. For a device to establish a communicating medium with another device, it first has to prove its identity to the server. It encrypts its ID with the public key of the server and sends it along with the details of the device it wants to connect. The server decrypts the message with its private key and provides the requested public key ( encrypted using server's private key ) after verifying the ID from the stored records. The devices then 
start to share messages as described in the communication protocol. The advantage of having a centralized server is, if a new participant is added to the network, instead of updating all the devices, only the server's database needs to be updated with the public keys and ID's of new entries. Thus, having a centralized server avoids unnecessary computations.

DEVICE A

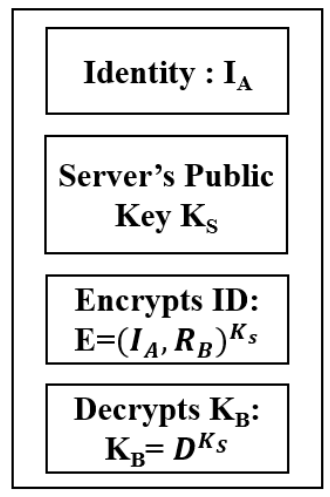

SERVER

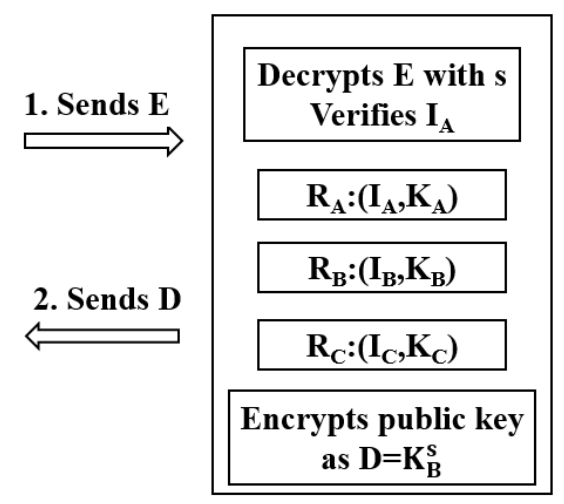

DEVICE B

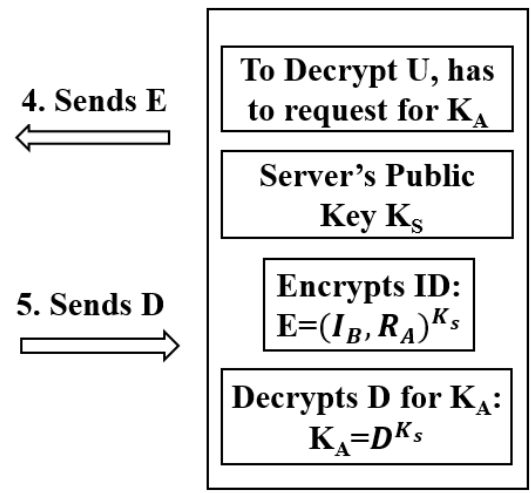

3. Sends the message $Y, Y_{B}, U$

\section{Communicating platform established}

Figure 4-4: Centralized Server

In Fig. 4-4 the authentication protocol between two devices is shown. The steps in the protocol are as follows:

1. Device A encrypts its ID $\left(I_{A}\right)$ and the name of the user it wants to connect $\left(R_{B}\right)$, with the public key $\left(K_{S}\right)$ of the server.

2. The server decrypts the encrypted message (E) with its private key s. Verifies the ID from records, encrypts the public key of device $\mathrm{B}\left(K_{B}\right)$ with its private key and sends (D) to A.

3. Device A decrypts (D) using server's public key $\left(K_{S}\right)$ and obtains $K_{B}$. Now, using the communication protocol, A sends the encrypted parameters $\mathrm{Y}, Y_{B}$ and $\mathrm{U}$ to device $\mathrm{B}$ (final step of the communication protocol is shown here). 
4. Device B encrypts its ID $\left(I_{B}\right)$ and sends it to the server as it needs public key of device $\mathrm{A}\left(K_{A}\right)$ to decrypt the hash $(\mathrm{U})$.

5. The server then verifies the ID and provides the encrypted public key (D).

6. The devices thus establish a communicating medium. 


\section{Chapter 5}

\section{Performance and Implementation}

\subsection{Security Analysis}

The Man In the Middle (MIM) attack allows the malicious or unauthorized users to access data through the backdoor [17]. Let us consider the following scenario as an example. If the attacker obtains the identity of device $\mathrm{A}$, he can request the server as device A to establish a connection with device B as shown in Fig. 5-1. The server provides the public key since the verification results are correct. Device B blindly trust the attacker as the trusted third party approves him as an authorized user. In this case, device $\mathrm{B}$ is highly prone to the threat of disclosing information to a malicious user.

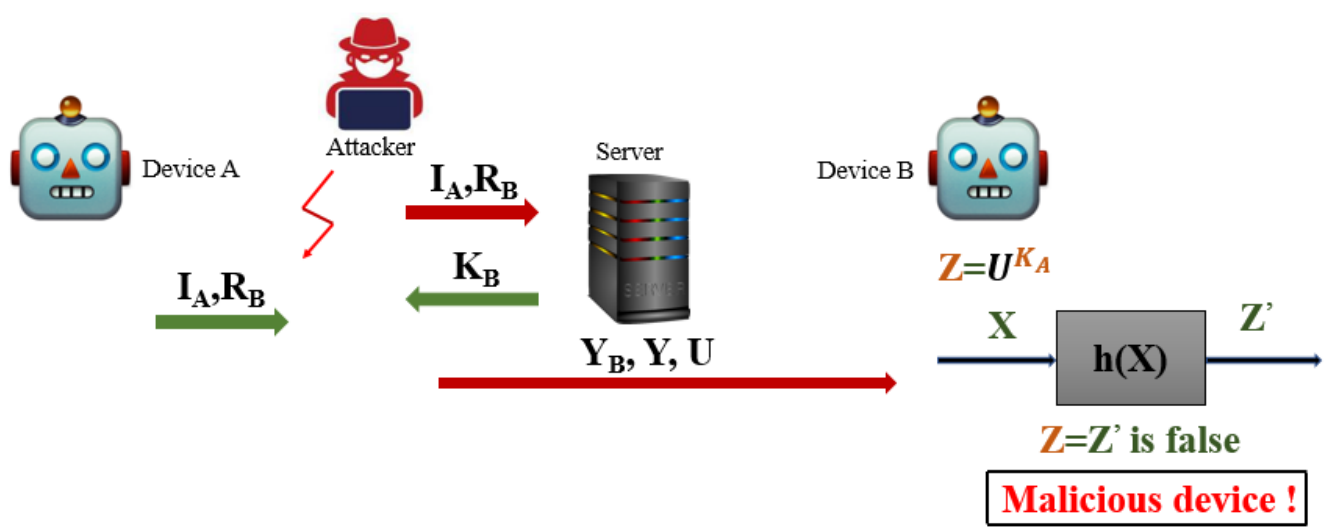

Figure 5-1: Attack Scenario 
Table 5.1: Security Services offered by the Protocol

\begin{tabular}{|l|l|l|}
\hline MIM Attacks & Security & Feature \\
\hline ID's & Unique numbers & Confidentiality \\
\hline Spoofing ID's & Server's records & Authentication \\
\hline Authorized ID's & Server's Public Key & Digital Signature \\
\hline Authentication & Hash Functions & Integrity \\
\hline Encryption Protocol & Gate Functions & Complexity \\
\hline
\end{tabular}

The customized protocol can tackle the attack this way: When the device B needs to decrypt the hash of the message, it requests the server for the public key of Device A. Server provides the public key $K_{A}$ to B. Unless the attacker encrypts the hash with the private key of A or replaces the public key sent by the server with his public key, it is less likely the verification turns out to be true. Thus, even when the attacker obtains authorization from the server, it is hard to run the attack successfully. This way, the integrity of the message is ensured.

Similarly, the different security features offered by the protocol are displayed in the Table 5.1. A unique ID is assigned to every device to ensure confidentiality. The server can easily identify the unauthorized users trying to spoof their identities. One of the strongest features of the protocol is that, to connect to the server, the user needs to possess the public key of the server which is pre-stored in all the participating devices as described in the authentication protocol. The feature acts as a digital certificate [19] on a small scale as a trusted third party legally approves all the devices as authorized users.

Let us assume man-in-the-middle obtained the authorization and could replace his public key in place of the intended public keys. He has all the standard encryption algorithms including the algorithm which works with sixteen polynomial equations and is trying for one of the algorithms to cooperate with the keys he possesses. Unaware of the additional layer used, it is difficult for unauthorized users to run the attacks successfully. The complex feature of the algorithm makes it impossible 
to determine the encryption technique used. Therefore from the Table 5.1, it is understood even if one of the services is compromised, the other acts as an alternative mechanism in obstructing the attacks.

\subsection{Algorithm Implementation}

The thesis laid out design analysis of the proposed protocol so far. In this section, we display the implementations of the algorithms in Python. Using the following logics and libraries [4] we could implement the customized approach in blocks.

\subsubsection{Key Generation}

Fig. 5-2 displays the generation of private and public keys. 1024-bit sized keys have been generated using RSA crypto library. The keys are directly stored in the created files.

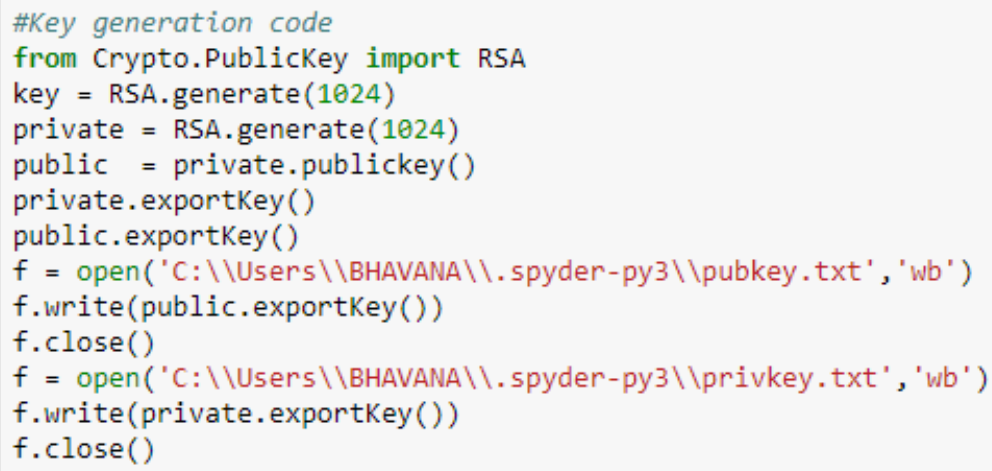

Figure 5-2: Keys Generation

\subsubsection{Hash Generation}

The generation of a hash using SHA-256 is displayed in the Fig. 5-3. The input to be hashed is the message, the sender is planning on sending. Further, the hash is 
encrypted using the private key.

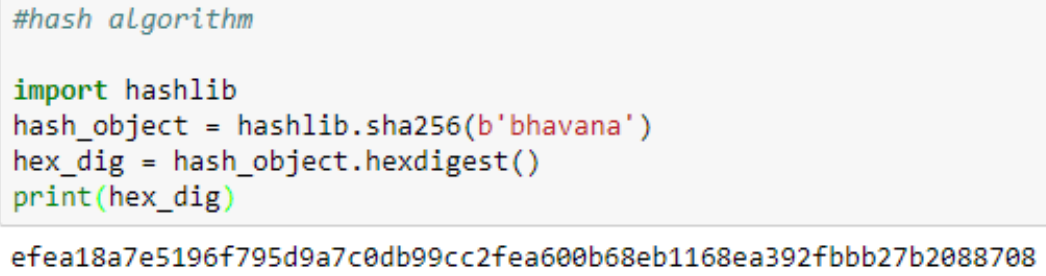

Figure 5-3: Hash Generation

\subsubsection{Encryption and Decryption}

The implementation of encrypting the hash with the private key is presented in the Fig. 5-4. Initially, message decrypted using the customized algorithm is resend as input to the hash function. The receiver decrypts hash from the sender with the shared public key and compares the computed and decrypted hash functions.

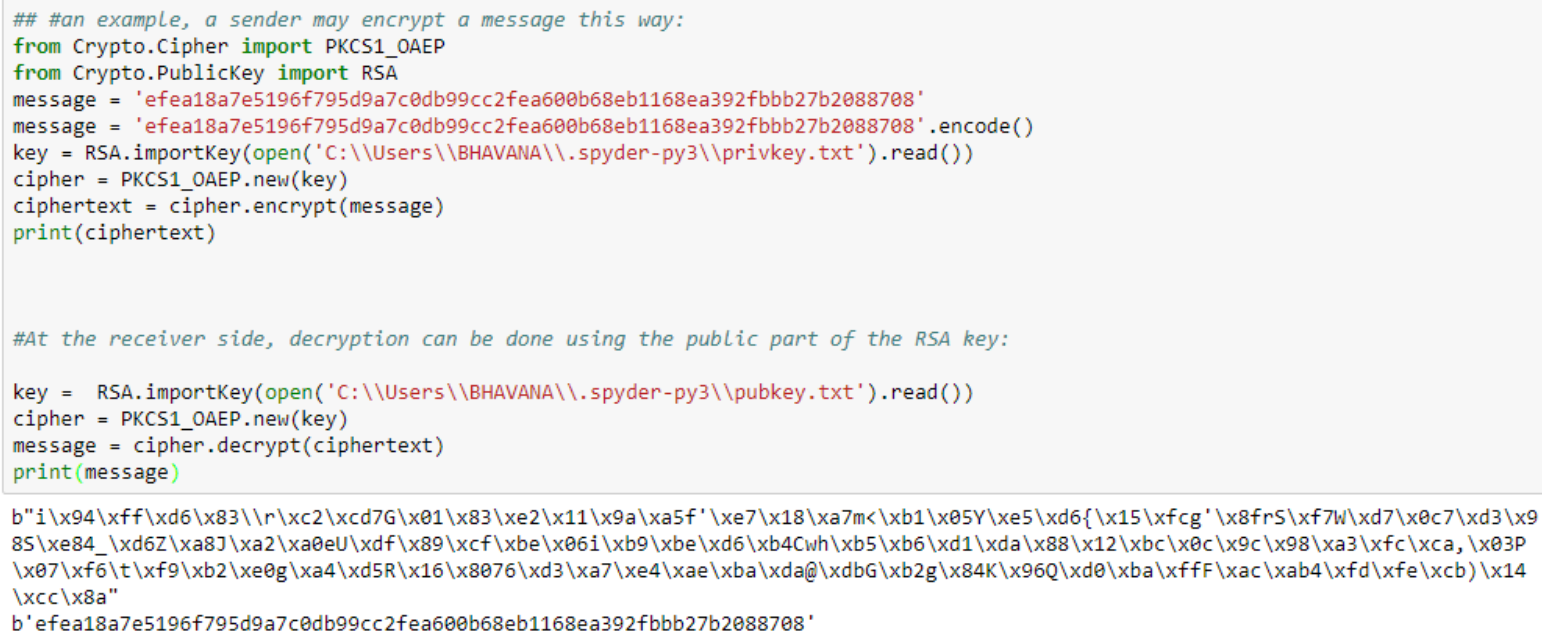

Figure 5-4: Encryption and Decryption of Hash 


\subsubsection{Gate Function}

We know the inputs to the gate function are the coefficients of the sixteen equations. The coefficients are taken as 16 string inputs into a list (a). The other input to the gate is assigned to another variable (b). An XOR operation performed between $\mathrm{a}$ and $\mathrm{b}$ generates 16 output strings as shown in Fig. 5-5

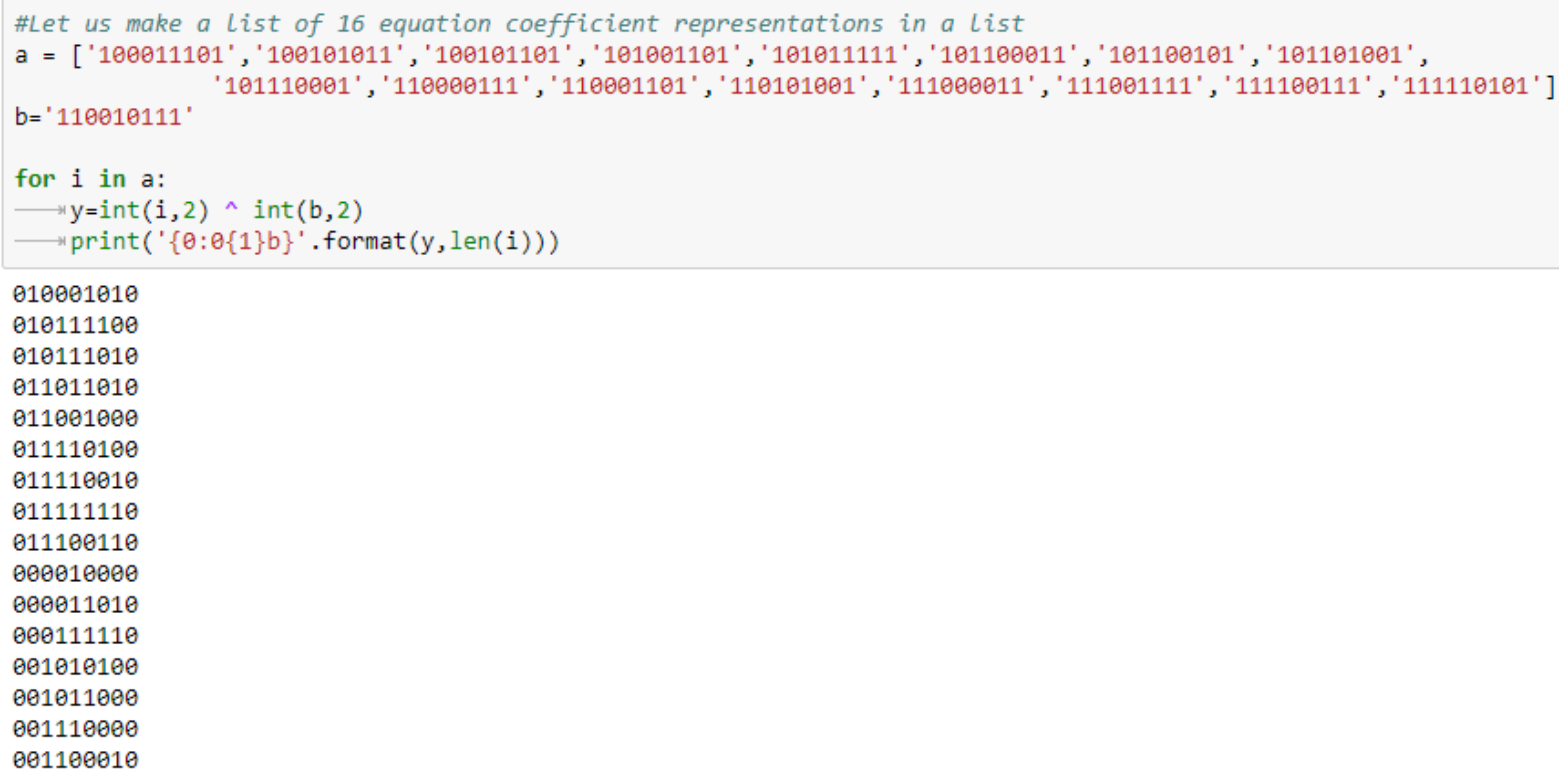

Figure 5-5: Gate Function

\subsubsection{Key Selection}

Fig. 5-6 demonstrates the generation of the 512-bit number and selection of the 128-bit key for the algorithm. Technically, once the number is generated, the output bits of gate function must be compared to every 9-bit of the random number, one after the other. In our implementation, we take the 9th output of the gate function to explain the selection process. The search for this output bit throughout the random number takes place. The match of string occurred at index 266. Therefore the bits from index 267 to 395 are chosen as the 128-bit key to customized encryption 
algorithm.

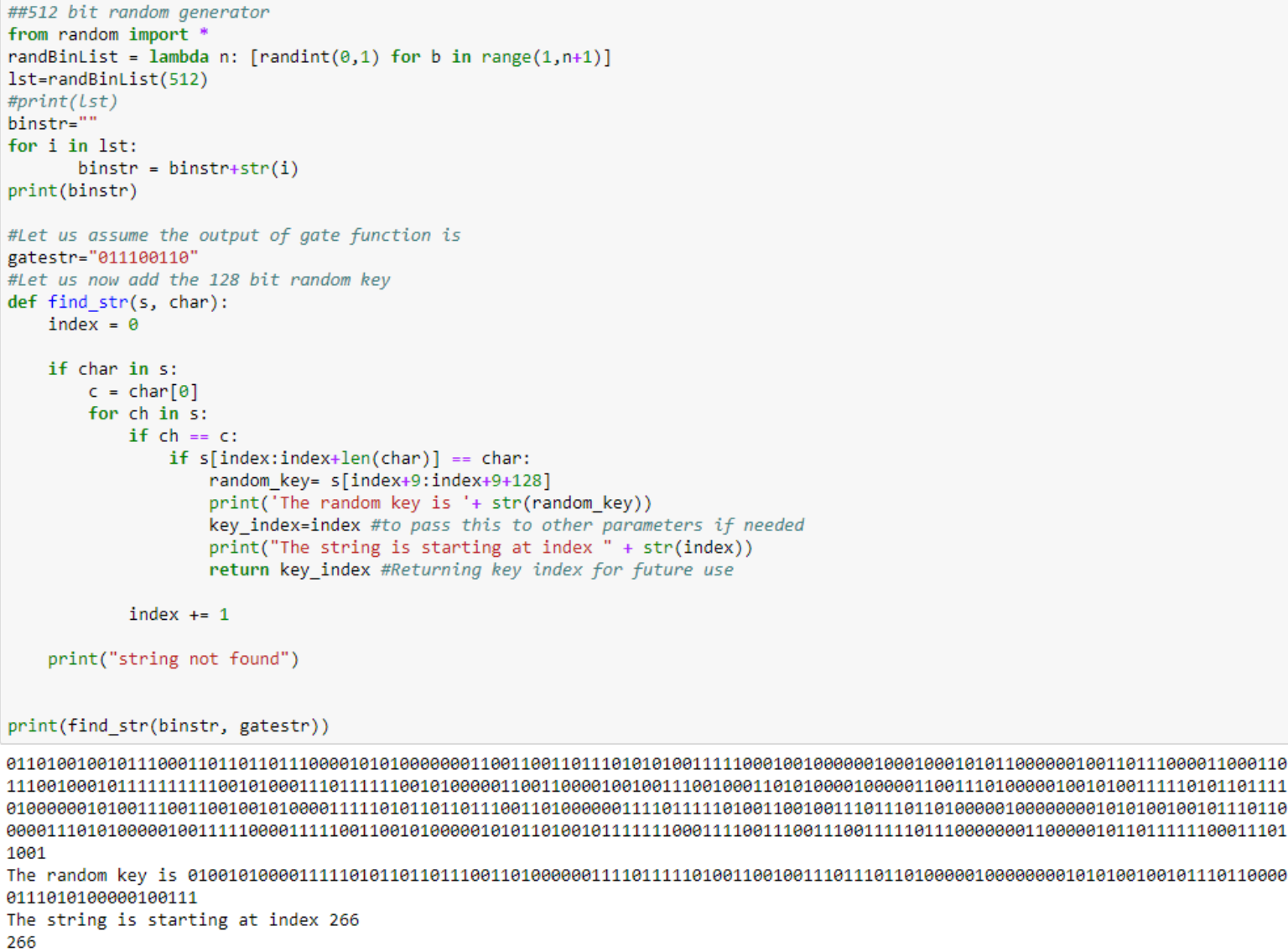

0110100100101110001101101101110000101010000000110011001101110101010011111000100100000010001000101011000000100110111000011000110 1110010001011111111110010100011101111110010100000110011000010010011100100011010100001000001100111010000010010100111110101101111 0100000010100111001100100101000011111010110110111001101000000111101111101001100100111011101101000001000000001010100100101110110 0000111010100000100111110000111110011001010000010101101001011111110001111001110011100111110111000000011000001011011111100011101 1001

The random key is 0100101000011111010110110111001101000000111101111101001100100111011101101000001000000001010100100101110110000 0111010100000100111

The string is starting at index 266

266

Figure 5-6: Key Selection

\subsubsection{Customized Encryption}

The AES algorithm obtained from the python library was modified to fit in our requirements. The encryption and decryption of the file were tested by importing the computed S-boxes and inverse S-boxes one after the other. These boxes are stored in a file AES_base and are imported from it. The implementation of the algorithm using the second S-box and Inverse S-box is shown in the Fig. 5-7. The text file lorem.txt shown in the Fig. 5-8 is taken as the plaintext to be encrypted. Our algorithm converts the 128-bits into 32 hexadecimal digits for convenience and uses them as the 


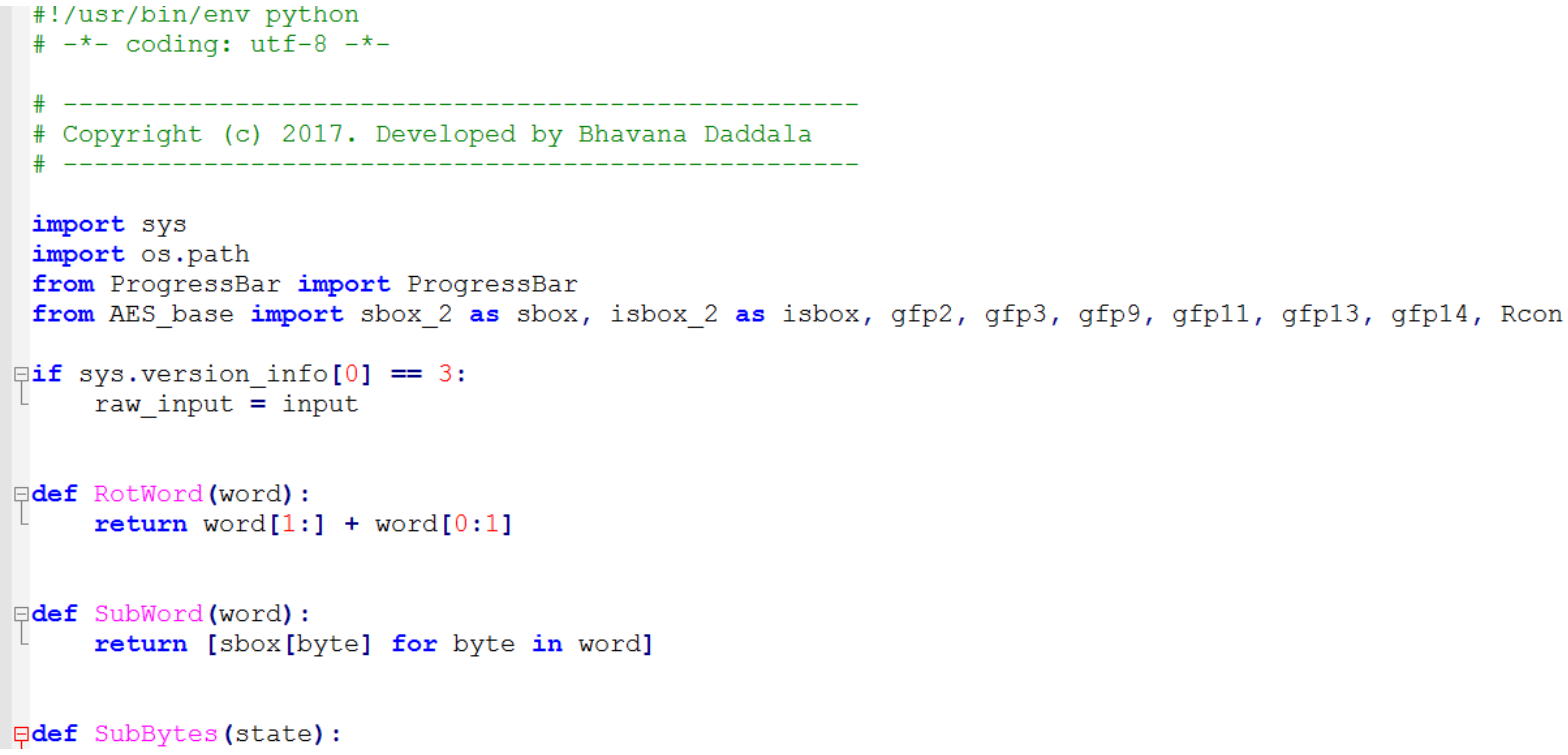

Figure 5-7: Importing selected S-box and Inverse S-box

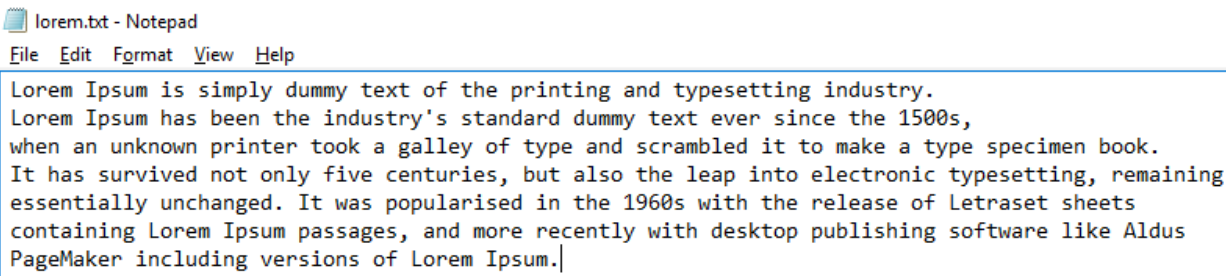

Figure 5-8: Plaintext

C:4. C:IWINDOWS $\backslash$ system $32 \backslash \mathrm{cmd}$.exe

\Users \welcome \Desktop \demo\AES \master \Python-AES >python AES.py -e lorem.txt

Enter a key, formed by 32 hexadecimal digits: 01101001001011100011011011011100001010100000001100110011011100110111 Key is too long. Keeping only the first 32 digits.

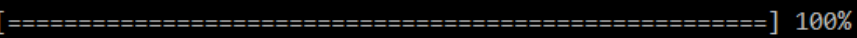

: \Users \welcome \Desktop \demo\AES \master \Python-AES〉.

Figure 5-9: Customized Encryption 
Figure 5-10: Ciphertext

6:- C: $\backslash$ WINDOWS $\backslash$ system $32 \backslash \mathrm{cmd}$.exe

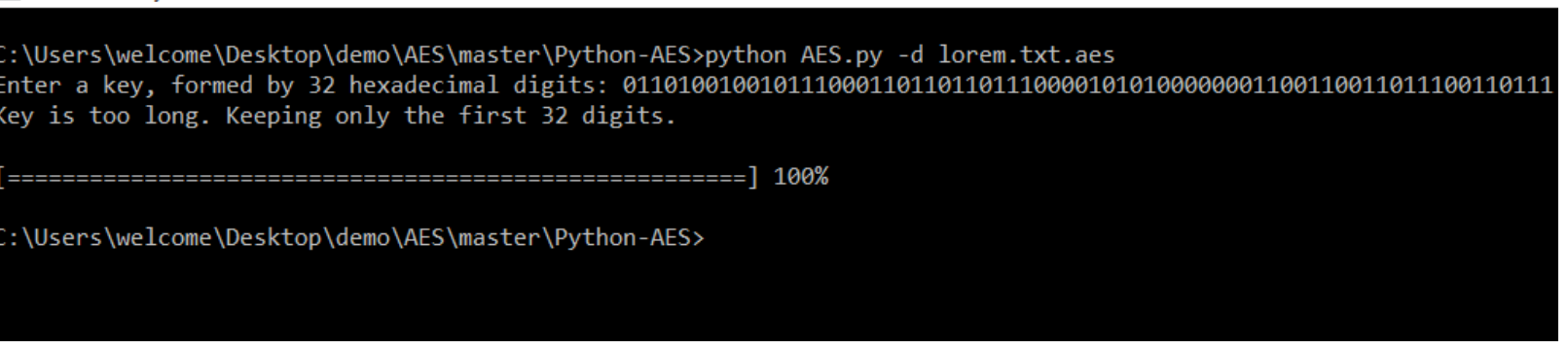

Figure 5-11: Decryption

key. The command -e lorem.txt shown in Fig. 5-9 is used to encrypt the file. The texture of an encrypted text is presented in Fig. 5-10. On the receiving end, the device uses the same protocol to derive the key and decrypts the message. As per our programming, the command -d lorem.txt decrypts the text file as shown in Fig. 5-11. The decryption end is created in such way that, it straight away creates a notepad file with decrypted text as shown in Fig. 5-12.

lorem.txt - Notepad

File Edit Format View Help

Lorem Ipsum is simply dummy text of the printing and typesetting industry. Lorem Ipsum has been the industry's standard dummy text ever since the 1500s, when an unknown printer took a galley of type and scrambled it to make a type specimen book. It has survived not only five centuries, but also the leap into electronic typesetting, remaining essentially unchanged. It was popularised in the 1960s with the release of Letraset sheets containing Lorem Ipsum passages, and more recently with desktop publishing software like Aldus PageMaker including versions of Lorem Ipsum.

Figure 5-12: Decrypted Text 


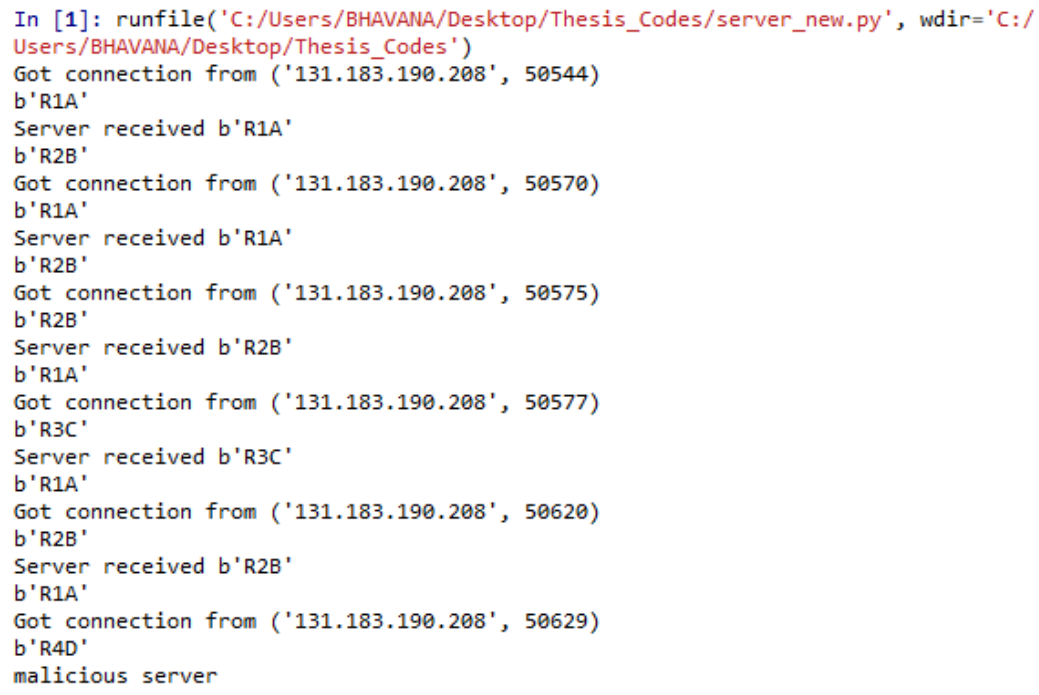

Figure 5-13: Server

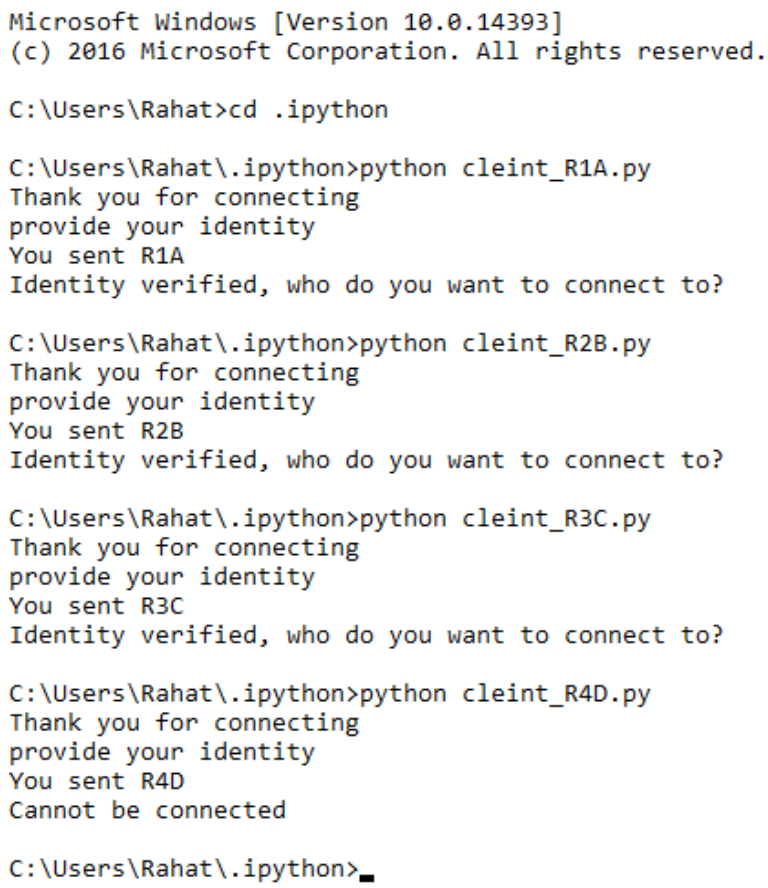

Figure 5-14: Client 


\subsubsection{Client-Server Environment}

The centralized distribution of keys has been created using the Client-Server environment [2]. The server acts as the trusted third party, and all the devices are considered as clients. We implemented the environment on two individual systems, i.e., executing the server on one system and client on the other system. The user names we assigned to devices are $\mathrm{R} 1 \mathrm{~A}, \mathrm{R} 2 \mathrm{~B}$, and $\mathrm{R} 3 \mathrm{C}$, which are saved in the dataset of the server as well. When client A wants to connect to the client B, it first connects to the server using the server's static IP. The server asks for identity and upon verifying the identity sends the public key of the requested device. The server sends the public key as a file, and the same file is created on the receiving end. If the server gets multiple requests at a time, it provides access on a random basis. The implementation of Client and server environment in Python is shown in the Fig. 5-13 and Fig. 5-14. The example of how the server detects a device (malicious) trying to connect with a fake identity is demonstrated as well. 


\section{Chapter 6}

\section{Conclusion and Future Work}

\subsection{Limitations}

In our implementation, the private and public keys of the devices are determined only once. Therefore, the devices don't need approval from the server every time they want to communicate. Once they have the public keys shared by the server, they are authorized. The consequence of this is, if the system is prone to compromise the keys after establishing a connection, the centralized server no longer provides help. The case is less likely to happen as hash functions still ensure non-repudiation. However, it is more beneficial to generate keys on a regular basis and update the server with new public keys of the devices. One other limitation is that the algorithm involves lots of computations resulting in longer execution times as compared to the standard methods.

An ideal encryption algorithm tries to provide maximum system security but can still be prone to attacks beyond prediction. The proposed protocol has been designed to be as secure as possible. An added gate layer is one of the biggest advantages of this algorithm. However, if the attacker manages to penetrate through this complex layer by any means, the algorithm is prone to vulnerabilities as any other security algorithms in the market. 


\subsection{Conclusion}

The necessary resources such as sixteen S-boxes, inverse S-boxes, multiplicative inverse tables required for the working of customized algorithm were implemented using Python. The key generation and selection protocol have been programmed in Python as well. The acquired tables used with AES framework resulted in text encryption and decryption. In a client-server environment, we implemented our centralized distribution of public keys. The RSA and SHA-256 algorithms imported from standard libraries were used to compute private keys, public keys, and hash functions.

Involving Internet of Things (IoT) in cryptography is an emerging domain. One of the core elements in securing an IoT infrastructure is around device identification and mechanisms to authenticate it [6]. Consequently, authentication and authorization will require appropriate re-engineering to accommodate the new IoT connected world. The proposed algorithm might find advantageous applications in the IoT since new authentication schemes that can be built using the experience of the existing encryption/authentication algorithms are required. In addition to this, it can achieve prominence in secure robotic communications as well [16]. Overall, the algorithm aims to provide a highly secure and stable encryption. Evaluating and optimizing the computational capabilities of the proposed algorithm could be one of the cases used for further research.

\subsection{Contributions}

Paper Publication: Design and Implementation of a Customized Encryption Algorithm for Authentication and Secure Communication between Devices in NAECON 2017 (National Aerospace \& Electronics Conference).

Won Best Poster Award at NAECON-2017 for the poster presented on "Design and Implementation of a Customized Encryption Algorithm for Authentication and 
Secure Communication between Devices". 


\section{References}

[1] Applications of Modern cryptography Technologies, applications and choices. https://www.surf.nl/binaries/content/assets/surf/en/knowledgebase/ 2010/rapport_201009_SNcryptoWEB.pdf Accessed July 13, 2017.

[2] Client/Server Environment. http://ccm.net/contents/ 152-client-server-environment Accessed July 16, 2017.

[3] Data Encryption Standard. http://searchsecurity.techtarget.com/ definition/Data-Encryption-Standard Accessed May 12, 2017.

[4] Python Libraries. https://www.python.org/ Accessed July 16, 2017.

[5] RSA algorithm (Rivest-Shamir-Adleman). http://searchsecurity. techtarget.com/definition/RSA Accessed May 12, 2017.

[6] Securing the Internet of Things: A Proposed Framework. http://www. cisco. com/c/en/us/about/security-center/secure-iot-proposed-framework . html Accessed July 13, 2017.

[7] What is The Data Encryption Standard (DES)? http://study.com/academy/ lesson/what-is-the-data-encryption-standard-des.htmlAccessed July $16,2017$.

[8] Martín Abadi and Roger Needham. Prudent engineering practice for cryptographic protocols. In Research in Security and Privacy, 1994. Proceedings., 1994 IEEE Computer Society Symposium on, pages 122-136. IEEE, 1994. 
[9] Nabihah Ahmad, Rezaul Hasan, and Warsuzarina Mat Jubadi. Design of aes s-box using combinational logic optimization. In Industrial Electronics $\&$ Applications (ISIEA), 2010 IEEE Symposium on, pages 696-699. IEEE, 2010.

[10] Jean-François Blanchette. Burdens of proof: Cryptographic culture and evidence law in the age of electronic documents. MIT Press, 2012.

[11] Emmanuel Bresson, Olivier Chevassut, David Pointcheval, and Jean-Jacques Quisquater. Provably authenticated group Diffie-Hellman key exchange. In Proceedings of the 8th ACM conference on Computer and Communications Security, pages 255-264. ACM, 2001.

[12] David Canright. A very compact S-box for AES. In International Workshop on Cryptographic Hardware and Embedded Systems, pages 441-455. Springer, 2005.

[13] J Lawrence Carter and Mark N Wegman. Universal classes of hash functions. Journal of computer and system sciences, 18(2):143-154, 1979.

[14] Shashikala Channalli and Ajay Jadhav. Steganography an art of hiding data. arXiv preprint arXiv:0912.2319, 2009.

[15] Pawel Chodowiec and Kris Gaj. Very compact fpga implementation of the aes algorithm. In Ches, volume 2779, pages 319-333. Springer, 2003.

[16] HR Everett. Robotic security systems. IEEE Instrumentation \& Measurement Magazine, 6(4):30-34, 2003.

[17] Subodh Gangan. A review of man-in-the-middle attacks. arXiv preprint arXiv:1504.02115, 2015.

[18] John Kelsey, Bruce Schneier, and David Wagner. Key-schedule cryptanalysis of idea, g-des, gost, safer, and triple-des. In Advances in CryptologyCRYPTO96, pages 237-251. Springer, 1996. 
[19] Stephen C Koehler. Method and system for authenticating digital certificates issued by an authentication hierarchy, October 9 2001. US Patent 6,301,658.

[20] Homin K Lee, Tal Malkin, and Erich Nahum. Cryptographic strength of ssl/tls servers: current and recent practices. In Proceedings of the rth ACM SIGCOMM conference on Internet measurement, pages 83-92. ACM, 2007.

[21] Septimiu Fabian Mare, Mircea Vladutiu, and Lucian Prodan. Secret data communication system using steganography, aes and rsa. In Design and Technology in Electronic Packaging (SITME), 2011 IEEE 17th International Symposium for, pages 339-344. IEEE, 2011.

[22] Robert J McEliece. Finite fields for computer scientists and engineers, volume 23. Springer Science \& Business Media, 2012.

[23] Alfred J Menezes, Paul C Van Oorschot, and Scott A Vanstone. Handbook of applied cryptography. CRC press, 1996.

[24] Christof Paar and Jan Pelzl. Understanding cryptography: a textbook for students and practitioners. Springer Science \& Business Media, 2009.

[25] Ronald L Rivest, Adi Shamir, and Leonard Adleman. A method for obtaining digital signatures and public-key cryptosystems. Communications of the ACM, 21(2):120-126, 1978.

[26] Rainer A Rueppel. Stream ciphers. In Analysis and Design of Stream Ciphers, pages 5-16. Springer, 1986.

[27] Gurpreet Singh. A study of encryption algorithms (rsa, des, 3des and aes) for information security. International Journal of Computer Applications, 67(19), 2013. 
[28] Yosef Stein, Haim Primo, and Joshua A Kablotsky. Galois field multiplier system, July 20 2004. US Patent 6,766,345.

[29] Michael Trott and Štefan Porubskỳ. Extended euclidean algorithm. 2008.

[30] Evangelos A Yfantis and Ahmad Fayed. Authentication and secure robot communication. International Journal of Advanced Robotic Systems, 11(2):10, 2014.

[31] Yuliang Zheng. Digital signcryption or how to achieve cost (signature \& encryption) cost (signature) + cost (encryption). Advances in CryptologyCrypto'9\%, pages 165-179, 1997. 